University of Louisville

ThinkIR: The University of Louisville's Institutional Repository

Electronic Theses and Dissertations

$12-2017$

\title{
Chemoselective reagents for derivatization of trace--level volatile carbonyl compounds using a microreactor approach.
}

Mumiye A. Ogunwale

University of Louisville

Follow this and additional works at: https://ir.library.louisville.edu/etd

Part of the Chemistry Commons

\section{Recommended Citation}

Ogunwale, Mumiye A., "Chemoselective reagents for derivatization of trace--level volatile carbonyl compounds using a microreactor approach." (2017). Electronic Theses and Dissertations. Paper 2830. https://doi.org/10.18297/etd/2830

This Doctoral Dissertation is brought to you for free and open access by ThinkIR: The University of Louisville's Institutional Repository. It has been accepted for inclusion in Electronic Theses and Dissertations by an authorized administrator of ThinkIR: The University of Louisville's Institutional Repository. This title appears here courtesy of the author, who has retained all other copyrights. For more information, please contact thinkir@louisville.edu. 
CHEMOSELECTIVE REAGENTS FOR DERIVATIZATION OF TRACE-LEVEL

VOLATILE CARBONYL COMPOUNDS USING A MICROREACTOR APPROACH

\section{By}

Mumiye A. Ogunwale

B. Tech (Hons)., Federal University of Technology Akure, 2003

M.S., Tennessee State University, 2011

A Dissertation

Submitted to the Faculty of the

College of Arts \& Sciences of the University of Louisville in Partial Fulfillment of the Requirements

for the Degree of

Doctor of Philosophy in Chemistry

Department of Chemistry

University of Louisville

Louisville, Kentucky

December 2017 

CHEMOSELECTIVE REAGENTS FOR DERIVATIZATION OF TRACE-LEVEL VOLATILE CARBONYL COMPOUNDS USING A MICROREACTOR APPROACH By

\author{
Mumiye A. Ogunwale \\ B. Tech (Hons)., Federal University of technology Akure, 2003 \\ M.S., Tennessee State University, 2011 \\ A Dissertation Approved On \\ November 6, 2017 \\ by the Following Dissertation Committee:
}

Michael H. Nantz, Ph.D., Dissertation Director

Xiao-An Fu, Ph.D., Dissertation co-Director

Xiang Zhang, Ph.D.

Daniel J. Conklin, Ph.D.

Natali B. Richter. Ph.D. 


\section{DEDICATION}

To:

1. The love of my life, Omowunmi.

2. To those who showed me light when I sat helplessly in the dark. 


\section{ACKNOWLEDGMENTS}

I am profoundly grateful for the mentorship of my advisors, Prof. Michael H. Nantz and Prof. Xiao-An Fu especially for their guidance, support, and encouragement during the course of my $\mathrm{PhD}$ program. These are the best mentors one can possible have!

Special thanks all my dissertation committee members Prof. Xiang Zhang, Prof.

Daniel J. Conklin and Prof. Natali Richter for accepting to serve as members of my dissertation committee and for all the fruitful and valuable discussions we had regarding this research.

I also appreciate the help of the American Heart Association Tobacco Center of Regulatory Science Exposure Core especially Prof. Daniel J. Conklin and Ms. Whitney S. Theis, M.S., for their technical help during our collaboration especially in using the cigarette smoking robot and software.

I am particularly grateful to Chemistry Department and School of Interdisciplinary and Graduate Studies, for the award of pre-doctoral fellowship and the Institute for Molecular Diversity and Drug Design (IMD3), for financial support. 
I would like to thank Dr. Pawel Lorkiewicz of Center for Regulatory and Environmental Analytical Metabolomics for training me on FT-ICR-MS and Ben Hutcherson for his assistance with GC-MS.

My thanks also go to Dr. Nantz's former lab members especially Dr. Ralph Knipp, Dr. Stephanie Mattingly, Dr. MVR Raju and Dr. Sadakatali Gori for their help during our stay together. I also appreciate the help of both current and former members of Dr. Fu's research group, Dr. Mingxiao Li, Dr. Yizheng Chen, Dr. Zhenzhen Xie and Mr. Qi Li.

I would also like to thank Dr. Julia Aebersold, and Mr. Curtis McKenna of the Micro/Nano Technology Center at the University of Louisville for their help in microfabrication.

I cherish the friendship, encouragement and help of Dr. Kelly Mouapi, and Mr. Rene Ebule when the PhD program was getting tough.

I am also highly indebted to my church RCCG Agape House Nashville and RCCG agape house Louisville for their prayers and support. A special thank to RCCG Agape House, Nashville, Tennessee for Initiative for Academic Excellence Scholarship Award I was given in 2009 to study in the US.

My sincere appreciation also goes to my lovely wife, Omowunmi and our children, Oba and Olamide, for their unalloyed support and encouragement without which this $\mathrm{PhD}$ and dissertation would not have been possible.

Finally, I am very grateful to my Lord Jesus -the author and finisher of my faithfor his graciousness and mercy to me from my childhood till date. 


\begin{abstract}
CHEMOSELECTIVE REAGENTS FOR CAPTURE AND DERIVATIZATION OF TRACE-LEVEL VOLATILE ALDEHYDES AND KETONES USING A MICROREACTOR APPROACH
\end{abstract}

\author{
Mumiye A. Ogunwale
}

November 6, 2017

Detection and analysis of trace level volatile aldehydes and ketones has become a significant research frontier because of the applicability for environmental monitoring and assessment, noninvasive diseases diagnosis, and in food safety assessment for the US Food and Drug Administration. The number of derivatization reagents for detection of aldehydes and ketones has increased considerably over the last decade. However, the majority of these derivatization reagents are not efficient in derivatizing unsaturated carbonyl compounds due to the presence of electron withdrawing groups adjacent to the reactive functional moieties making them insufficiently nucleophilic.

The analysis of trace-level carbonyl compounds challenges existing analytical instrumentation because their concentrations are below current instrument limits of detection. 
This study shows for the first time the application of an innovative silicon-based microreactor for preconcentration of carbonyl compounds in electronic cigarette aerosols. The microreactor is coated with an aminooxy reagent, typically 4-(2-aminooxyethyl)morpholin-4-ium chloride (AMAH) or 2-(aminooxy)-N, N, N-trimethylethanammonium iodide (ATM). The aminooxy functional group chemoselectively traps trace aldehydes and ketones generated by aerosolization of electronic liquids by means of oximation reactions. The aminooxy-carbonly adducts and unreacted aminooxy reagent are eluted from the microreactor using $150 \mu \mathrm{L}$ of methanol followed by addition of an internal standard (for quantification) and then analyzed by Fourier transform ion cyclotron resonance (FT-ICR) mass spectrometry (MS) or gas chromatography mass spectrometry (GC-MS), depending on the capture reagent used.

Chapter 1 describes different methods of detection and analysis of volatile organic aldehydes and ketones in gaseous samples such as exhaled breath and electronic cigarette aerosols. Chapter 2 presents the analysis and quantification of carbonyl compounds in electronic liquid aerosols. Chapter 3 describes the kinetic studies of oximation reactions of the aminooxy reagents AMAH, ADMH and ATM. It also outlines the synthesis of a cationic hydrazine-based reagent for derivatization of aldehydes and ketones. Chapter 4 describes the quantification of nicotine in e-cig liquids and derived aerosols using both FT-ICR-MS and GC-MS. Chapter 5 provides the overall summary and future direction. 
TABLE OF CONTENTS

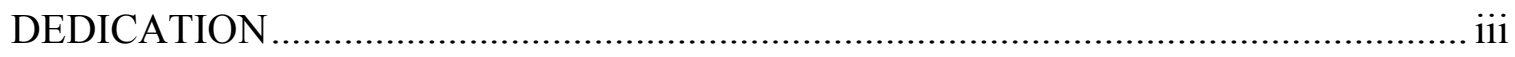

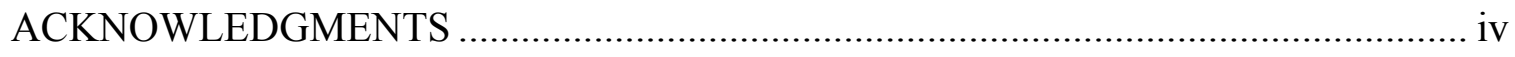

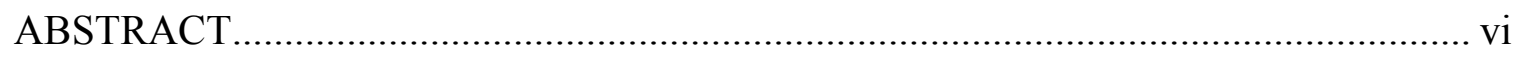

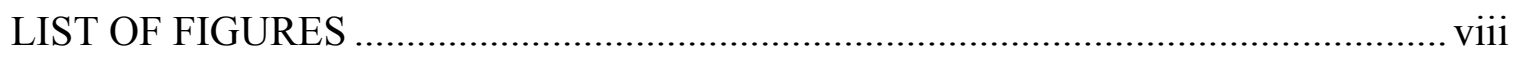

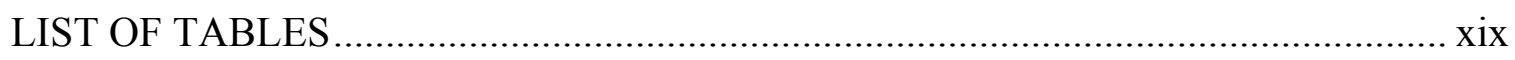

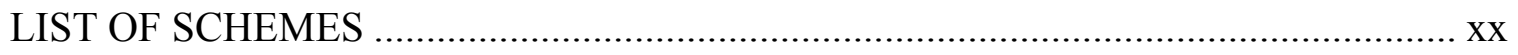

CHAPTER 1. DETECTION AND ANALYSIS OF VOLATILE ORGANIC

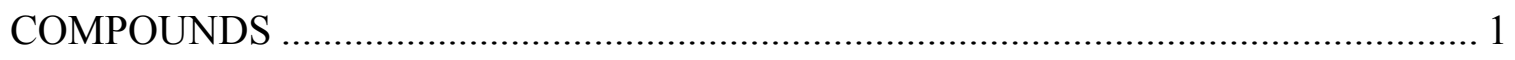

1.1. HYPOTHESIS AND CONTRIBUTION OF THIS DISSERTATION ............... 2

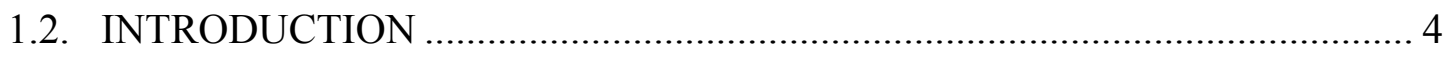

1.3. PRECONCENTRATION TECHNIQUES .................................................. 6

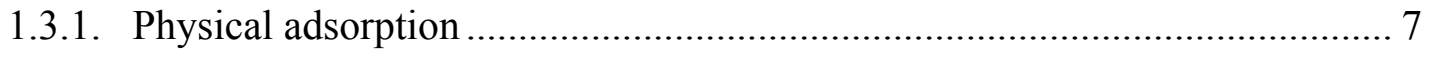

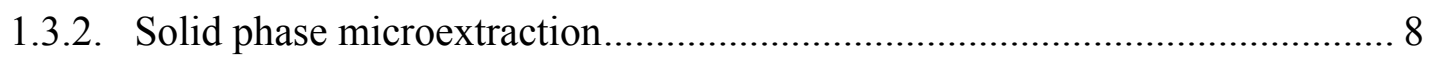

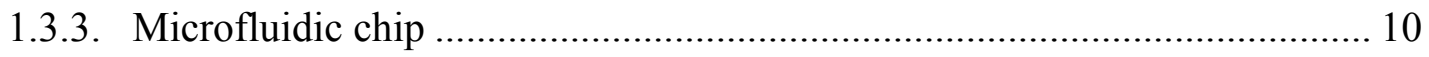

1.4 SURVEY OF DERIVATIZATION REAGENTS FOR CARBONYL

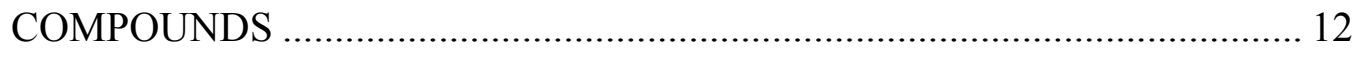

1.5. ANALYTICAL DETECTION TECHNIQUES .......................................... 14 
1.5.1. Gas chromatography mass spectrometry .............................................. 15

1.5.2. Liquid chromatography-tandem mass spectrometry (LC-MS/MS)............ 15

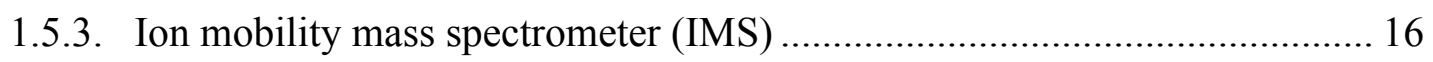

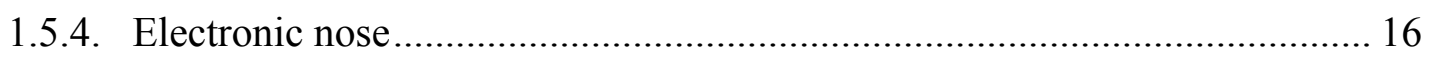

1.5.5. Fourier transform ion cyclotron resonance mass spectrometry (FT-ICR-

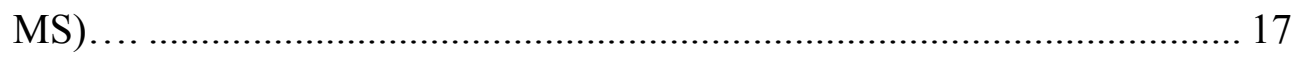

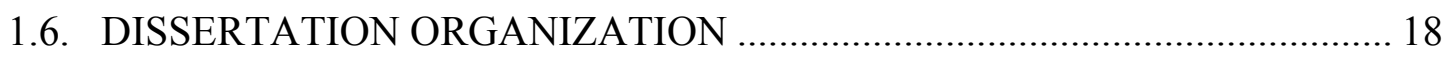

CHAPTER 2. CARBONYL COMPOUNDS IN ELECTRONIC LIQUIDS AND

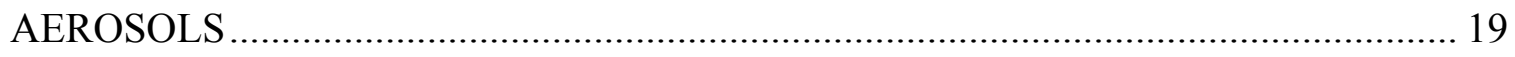

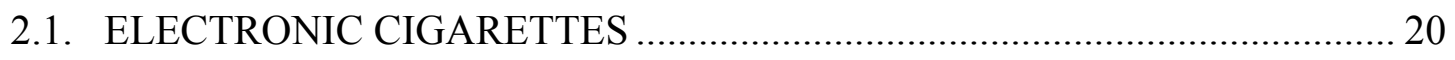

2.2. SIGNIFICANCE OF CARBONLYS IN ELECTRONIC CIGARETTES ........ 21

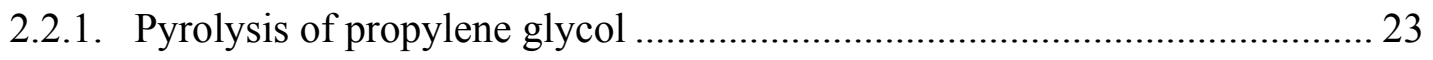

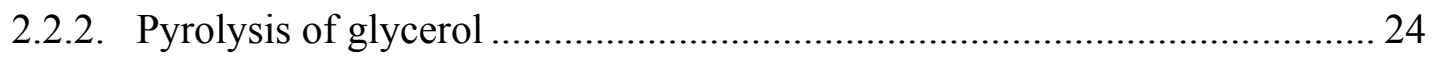

2.2.3. Hemiacetal formation in aerosols of e-liquids ...................................... 25

2.3. E-CIGARETTE AEROSOL GENERATION; CAPTURE, DERIVATIZATION AND ANALYSIS OF CARBONYL COMPOUNDS .................................. 26

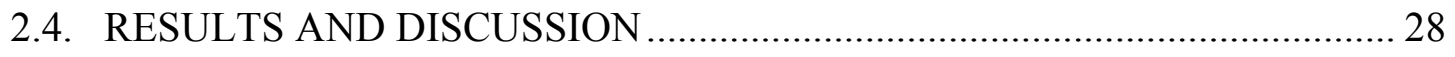

2.4.1. Carbonyls in aerosols of commercial e-liquids with flavorants.................. 28

2.4.2. Generation of carbonyls from e-cigarettes aerosols: effects of battery power

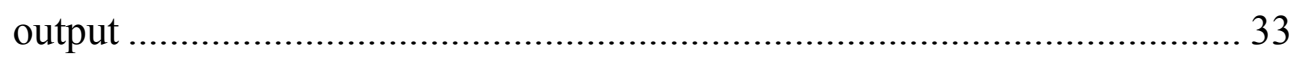

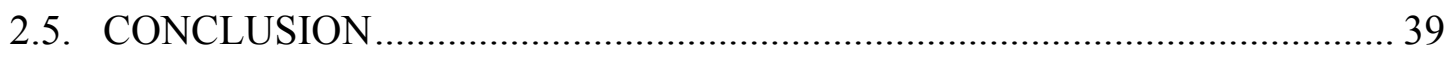

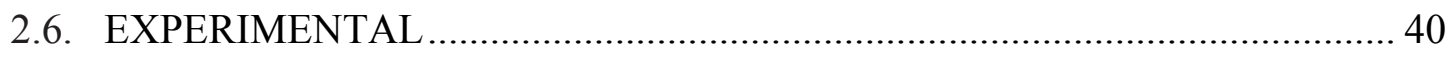




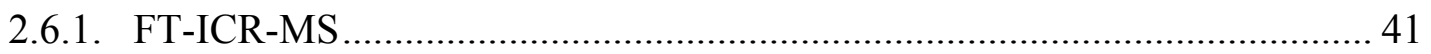

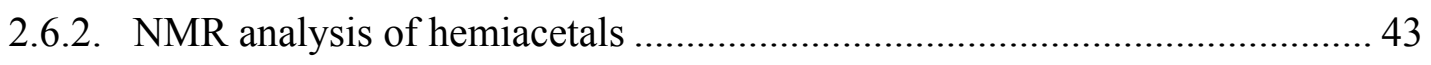

2.6.3. GC-MS analysis of carbonyl adducts ......................................................... 43

CHAPTER 3. KINETIC STUDIES OF OXIMATION REACTION AND SYNTHESIS

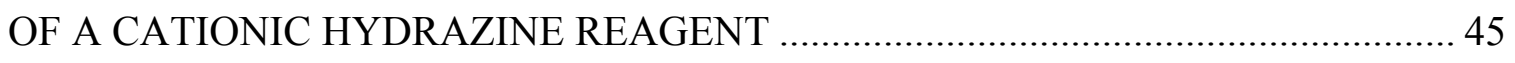

3.1. INTRODUCTION OF OXIMATION REACTIONS ........................................ 46

3.2. KINETIC STUDIES OF REACTION BETWEEN AMINOOXIES AND

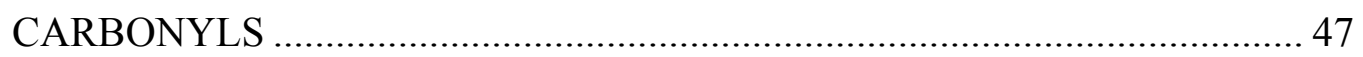

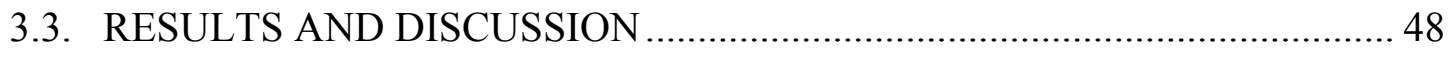

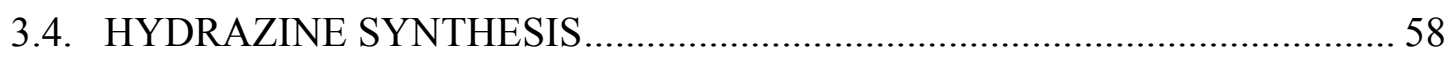

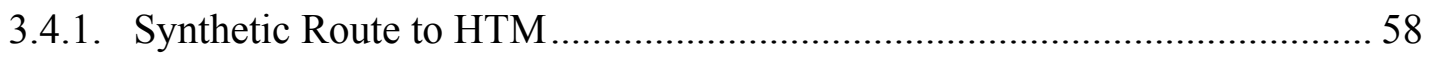

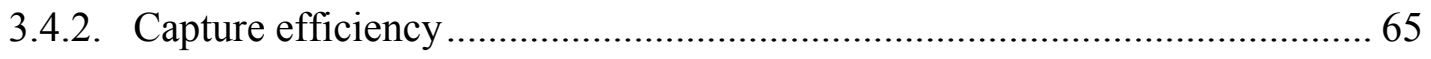

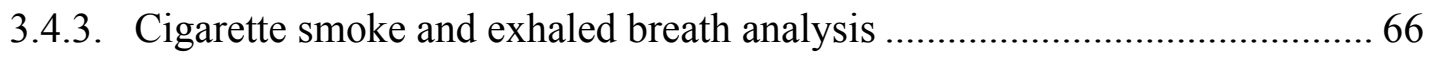

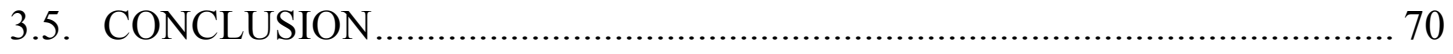

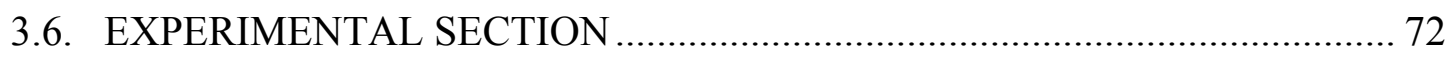

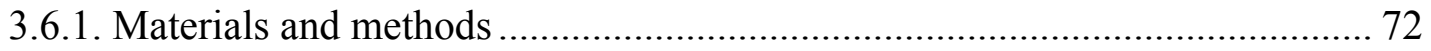

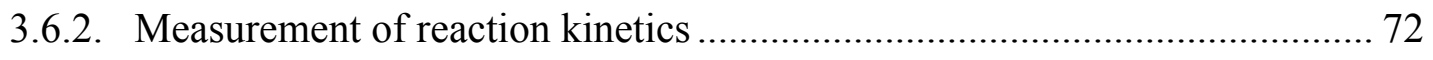

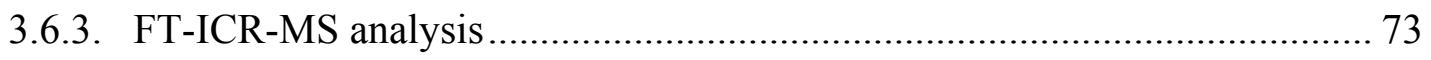

3.6.4. 2-Hydroxy-N, N, N-trimethylethan-1-ammonium iodide .............................. 73

3.6.5. 2-Chloro-N, N, N-trimethylethan-1-ammonium iodide................................ 74

3.6.6. 2-Hydrazinyl-N, N, N-trimethylethan-1-ammonium iodide (HTM) ............ 74

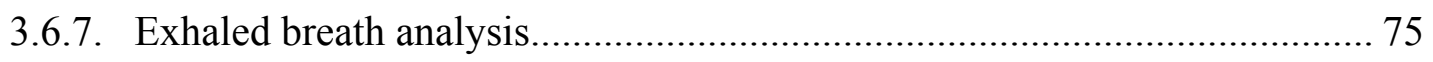


3.6.8. Cigarette smoke analysis

CHAPTER 4. NICOTINE IN E-CIG LIQUIDS AND DERIVED AEROSOLS........... 77

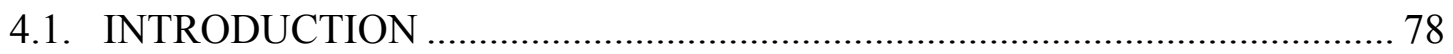

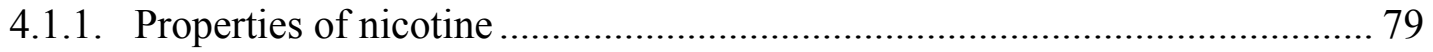

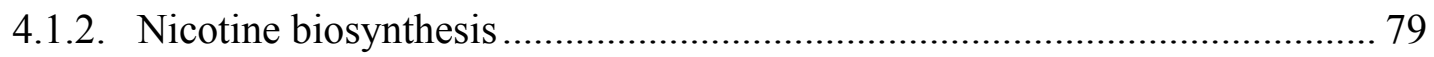

4.2. STANDARD METHOD FOR NICOTINE COLLECTION AND

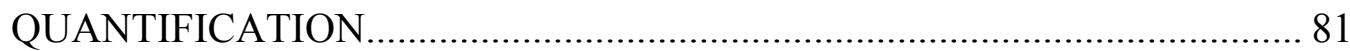

4.3. EXPERIMENTAL DESIGN FOR NICOTINE SAMPLE COLLECTION AND

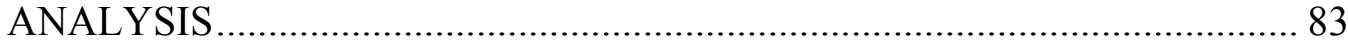

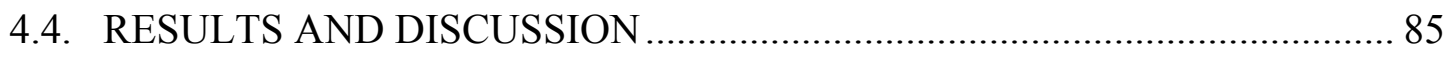

4.4.1. Measurement of nicotine kinetics of protonation by NMR spectroscopy .... 85

4.4.2. Calibration curve of protonated nicotine by FT-ICR-MS.......................... 90

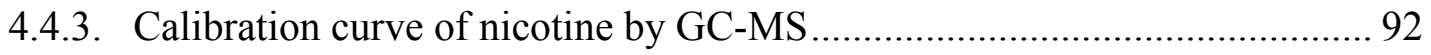

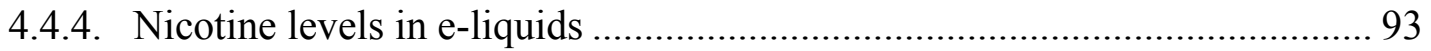

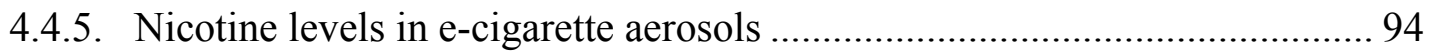

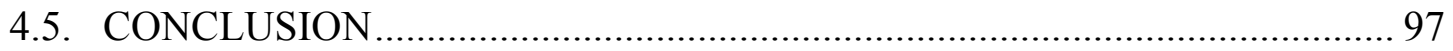

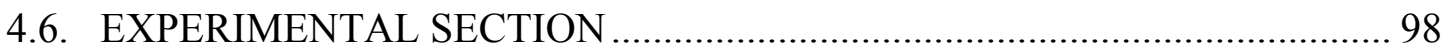

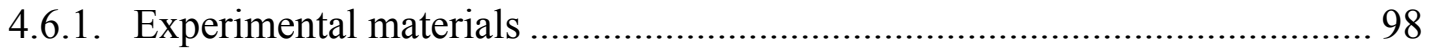

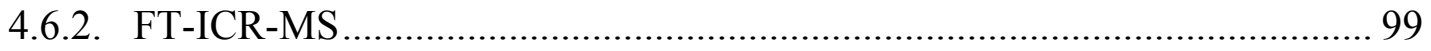

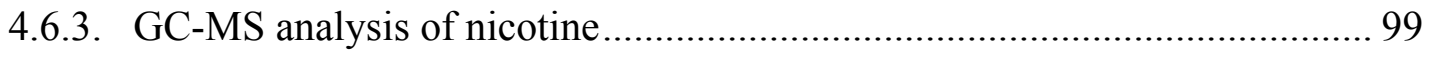

4.6.4. Measurement of nicotine NMR spectroscopy .......................................... 99

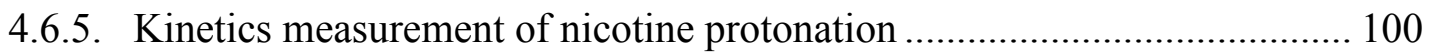




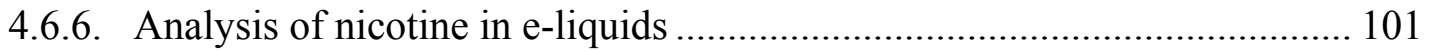

4.6.7. Collection and analysis of nicotine in e-cigarette aerosols ........................ 101

CHAPTER 5. SUMMARY AND FUTURE WORK ............................................. 104

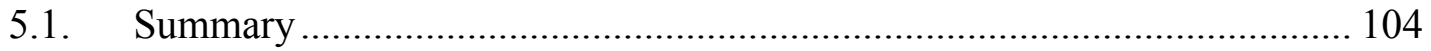

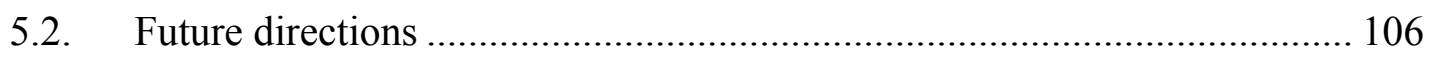

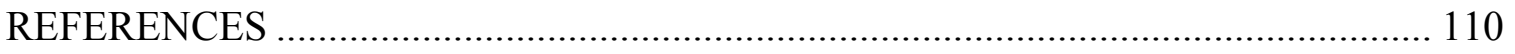

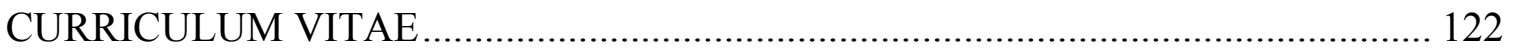




\section{LIST OF FIGURES}

Figure 1.0. The structures of ATM, AMAH, HTM and nicotinium chloride salt .......... 4

Figure 1.1. SPME procedure for GC and LC ....................................................... 9

Figure 1.2. 2-(Aminooxy) ethyl-N, N, N-trimethylammonium iodide (ATM)............ 11

Figure 1.3. Photographs of the microreactor ........................................................ 11

Figure 1.4. Reagent for derivatization of carbonyl compounds ............................... 13

Figure 1.5. DNPH cartridge for derivatization of carbonyl compounds ....................... 14

Figure 2.1. Anatomy of an electronic cigarette .................................................. 21

Figure 2.2. Cigarette-smoking robot system for mechanical generation of aerosols from

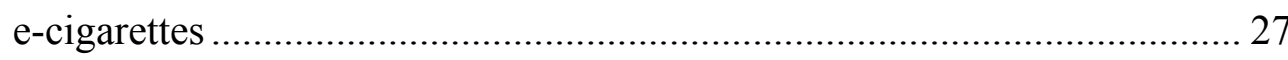

Figure 2.3. Schematic diagram of the preconcentration set-up for capture of carbonyl

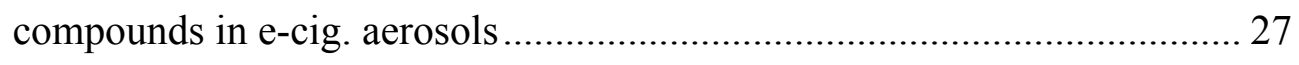

Figure 2.4. The FT-ICR-MS of the aerosols of $100 \% \mathrm{PG}, 100 \% \mathrm{VG}$ and PG/VG in $50 / 50$ ratio 31

Figure 2.5. Amounts of formaldehyde, acetaldehyde, acrolein and acetone as a function of the percentage of propylene glycol (PG) in the mixtures of vegetable glycerin (VG) and propylene glycol. 31

Figure 2.6. Amounts of formaldehyde, acetaldehyde, acrolein and acetone as a generated while vaping a set of e-cigarrettes as compared with neat PG and neat VG. 32 
Figure 2.7. Amounts of formaldehyde, acetaldehyde, acrolein and acetone generated while vaping another set of e-cigarrettes as compared with neat PG and neat VG 32

Figure 2.8. Representative GC-MS chromatograms of aldehydes generated fron Halo Mentol Ice at a battery power output of $11.7 \mathrm{~W}, 14.7 \mathrm{~W}$, and $16.6 \mathrm{~W}$ 34

Figure 2.9. ${ }^{1} \mathrm{H}$ NMR spectra (DMSO- $d_{6}$ ) for detection of hemiacetal for....................... 36

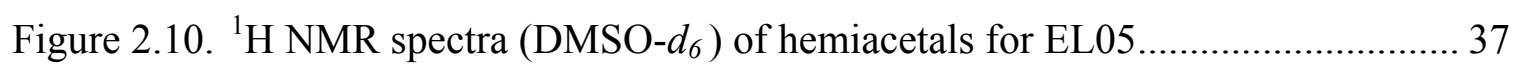

Figure 2.11. The relationship between the amounts of formaldehyde-hemiacetal in 10 puffs of aerosols collected from e-liquid EL05 .................................... 38

Figure 2.12. The calibration curves of standard AMAH-carbonyl compounds .............. 42

Figure 3.1. The plot of $1 / \mathrm{C}$ against time for oximation reaction between $\mathrm{AMAH}$ and acetone at $-21^{\circ} \mathrm{C}, 0{ }^{\circ} \mathrm{C}, 21^{\circ} \mathrm{C}$. 50

Figure 3.2. FT-ICR-MS spectra overlay of oximation reaction between AMAH and acetone at $21{ }^{\circ} \mathrm{C}$ at times $\mathrm{t}=60,30,600,900$, and 1200 seconds 51

Figure 3.3. The plot of $1 / \mathrm{C}$ against time for oximation reaction between $\mathrm{ADMH}$ and acetone at $-21^{\circ} \mathrm{C}, 0{ }^{\circ} \mathrm{C}, 21^{\circ} \mathrm{C}$. 51

Figure 3.4. Spectra overlay of oximation reaction between $\mathrm{ADMH}$ and acrolein at $21{ }^{\circ} \mathrm{C}$ at times $\mathrm{t}=60,30,600,900,1200$ seconds. 52

Figure 3.5. The graph of the natural logarithms of the reaction rate constants of oximation reaction as a function of the reciprocal of temperature between ADMH, AMAH, ATM and acetone. 52 
Figure 3.6. The graph of the natural logarithms of the rate constants of oximation reaction as a function of the reciprocal of temperature between $\mathrm{ADMH}$, AMAH, ATM and propanal............................................................... 55

Figure 3.7. The graph of the dependence of the natural logarithms of the rate constants of oximation reaction as a function of the reciprocal of temperature between ADMH, AMAH, ATM and acrolein. 55

Figure 3.8. The graph of the dependence of the natural logarithms of the rate constants of oximation reaction as a function of the reciprocal of temperature between ADMH, AMAH, ATM and crotonaldehyde......................................... 56

Figure 3.9. The graph of the dependence of the natural logarithms of the rate constants of oximation reaction as a function of the reciprocal of temperature between ADMH, AMAH, ATM and 2-heptanone........................................... 56

Figure 3.10. The graph of the dependence of the natural logarithms of the rate constants of oximation reaction as a function of the reciprocal of temperature between ADMH, AMAH, ATM and methyl isobutyl ketone (MIBK)................... 57

Figure 3.11. 2-Hydrazinyl- $N, N, N$ - trimethylethan-1-ammonium iodide (HTM).......... 58

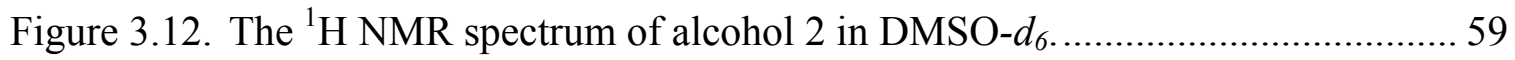

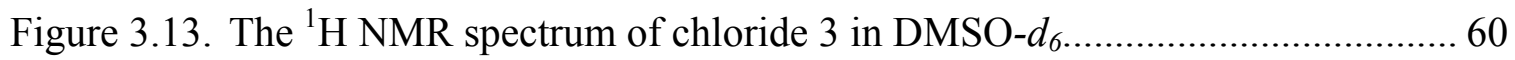

Figure 3.14. The high-resolution mass spectrometry spectra overlay of alcohol 2 and chloride 3. 60

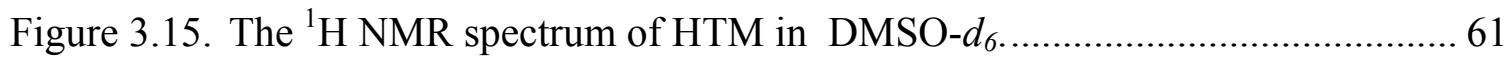

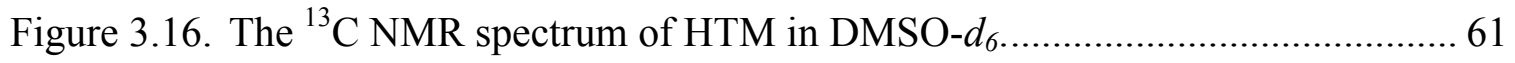


Figure 3.17. The FT-ICR-MS of the HTM and HTM-acetone- $d_{6}$ adduct (internal standard)

Figure 3.18. The graph of $1 / \mathrm{C}$ against time for hydrazone formation reaction between HTM and acrolein at $-21^{\circ} \mathrm{C}, 0{ }^{\circ} \mathrm{C}$, and $21{ }^{\circ} \mathrm{C}$ 63

Figure 3.19. The graph of the dependence of the natural logarithms of the rate constants of hydrazone formation reaction as a function of the reciprocal of temperature between HTM and propanal, acetone, acrolein, and crotonaldehyde. 63

Figure 3.20. The graph of the dependence of the natural logarithms of the rate constants of hydrazone formation reaction as a function of the reciprocal of temperature between ATM and propanal, acetone, acrolein, and crotonaldehyde 64

Figure 3.21. The experimental set-up showing the preconcentration of carbonyls in the microchip. 66

Figure 3.22. The capture efficiency graph of HTM and ATM with acrolein and crotonaldehyde. 66

Figure 3.23. The calibration curves of HTM-carbonyl adducts using HTM-acetone- $d_{6}$ adduct as internal standard 67

Figure 3.24. The calibration curves of ATM-carbonyl adducts using ATM-acetone- $d_{6}$ adduct as internal standard. 68

Figure 3.25. The FT-ICR-MS spectra overlay of HTM-carbonyl compounds detected in exhaled breath of a smoker and a non-smoker subjects 69

Figure 4.0. The structure of nicotine 78 


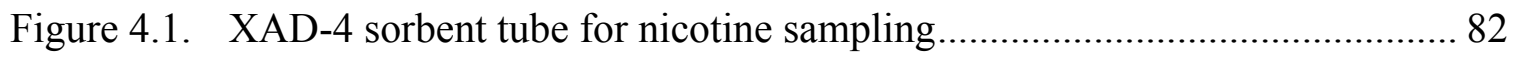

Figure 4.2. The schematic diagram of the inExpose Scireq smoking robot................... 84

Figure 4.3. Impinger optimization: capture efficiencies of 5 impingers connected in series 85

Figure 4.4. The plot of $\mathrm{n}($ nicotine $) / \mathrm{n}$ (benzene) vs. $\mathrm{H}$ (nicotine) $/ \mathrm{H}($ benzene $)$, serving as a calibration curve for ${ }^{1} \mathrm{H}$ NMR measurement of nicotine protonation.......... 86

Figure 4.5. ${ }^{1} \mathrm{H}$ NMR spectra (DMSO- $d_{6}$ ) of neutral nicotine and ${ }^{1} \mathrm{H}$ NMR spectra $\left(\mathrm{DMSO}-d_{6}\right)$ of extracted sample after 30 minutes of protonation in water and $\mathrm{HCl}$ mixture 87

Figure 4.6. Percent conversion of nicotine to its nicotinium salt at different temperatures over time 88

Figure 4.7. Dependence of $\ln \left[\right.$ nicotine] on the reaction temperature $\mathrm{T}\left({ }^{\circ} \mathrm{C}\right) \ldots \ldots \ldots \ldots . . . . . . .89$

Figure 4.8. The relationship between $\ln \mathrm{k}$ and $1 / \mathrm{T}$ for protonation of nicotine in $\mathrm{HCl}$ solution 89

Figure 4.9. The calibration curve of nicotine by plotting the ratio of intensity of nicotine-to-nicotine- $d_{3}$ (I-Nic/I-Nic- $\left.d_{3}\right)$ against the ratio of the amounts (mole) of nicotine-to-nicotine- $d_{3}\left(\mathrm{M}-\mathrm{Nic} / \mathrm{M}-\mathrm{Nic}-d_{3}\right)$ 91

Figure 4.10. Comparison of FT-ICR-MS spectra of standard calibration curve working solutions, each spiked with $7.78 \mathrm{nmol}$ nicotinium- $d_{3}$ as an internal standard.

Figure 4.11. The calibration curve of nicotine built by plotting ratio of mole of nicotineto-quinoline against the ratio of peak area of nicotine-to-quinoline. 93 
Figure 4.12. Comparison of GC chromatogram of standard calibration curve working solutions 93

Figure 4.13. Nicotine delivery profile of e-cigarette cartridges and e-liquids with different nicotine levels..................................................................... 96

Figure 4.14. The relationship between nicotine aerosol and nicotine levels in e-liquids at constant puff numbers................................................................... 97

Figure 5.0. ATM, ADMH, AMAH, HTM and nicotinium chloride salt.................... 104 


\section{LIST OF TABLES}

Table 1.0. Adsorbent materials and their composition ............................................ 8

Table 2.1. Physical properties of propylene glycol (PG) and vegetable glycerol (VG) 30

Table 2.2. Effect of varying battery power output on generation of aldehydes............ 35

Table 2.3. Characteristics of e-cigarette cartridges and e-liquids used in this study .... 36

Table 3.1. The activation energies and frequency factor of AMAH, ADMH, and ATM reactions with acetone, propanal, 2-heptanone, MIBK, acrolein and

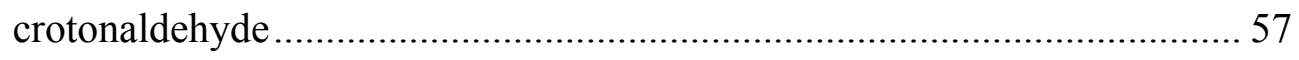

Table 3.2. Activation energies of HTM and ATM adducts of carbonyl compounds ... 65

Table 3.3. Carbonyl compounds quantified from 3R4F cigarette smoke ..................... 68

Table 3.4. The average and standard deviation of carbonyl concentrations of gaseous breath samples

Table 4.0. Results of nicotine analysis from selected commercial e-liquids ............... 94

Table 4.1. Comparison of measurements of nicotine in aerosol samples collected by sorbent tube and impinger methods 


\section{LIST OF SCHEMES}

Scheme 1.0. Formation of hydrazones and oximes by reaction of alpha nucleophiles with

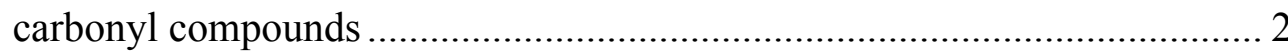

Scheme 1.1. Condensation reactions of aminooxy reagent ATM and hydrazine reagent HTM with aldehydes and ketones................................................... 3

Scheme 1.2. Derivatization of carbonyl compounds with Girard's reagent T and AMAH 13

Scheme 2.0. The homogeneous oxidation routes of propylene glycol.......................... 23

Scheme 2.1. Possible reactions occurring in glycerol pyrolysis.................................. 25

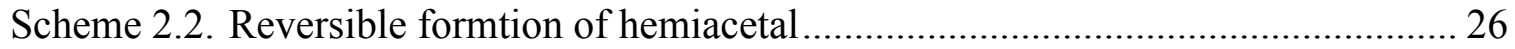

Scheme 2.3. Proposed pyrolysis of vegetable glycerol (VG) and propylene glycol (PG) humectants inelectronic cigarette aerosols............................................. 30

Scheme 2.4. Microreactor oximation of carbonyl compounds by AMAH .................... 41

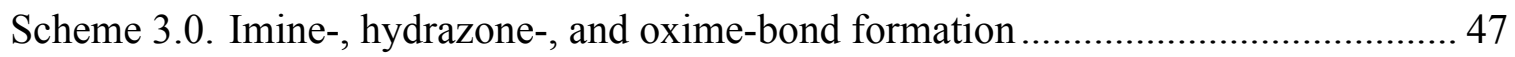

Scheme 3.1. Quaternary ammonium aminooxy reagents and oximation of aldehydes or

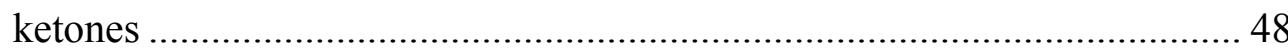


Scheme 3.2. Synthesis of ADMH reagents and conditions.....

Scheme 3.3. Synthesis of HTM, reagents and conditions

Scheme 3.4. Resonance structure of acrolein

Scheme 4.0. Biosynthetic pathway of nicotine. 81

Scheme 4.1. Regioselective protonation of nicotine to form the nicotinium salt............ 83

Scheme 5.0. Oxidative N-nitrosation of nicotine to form NNK and NNA 107

Scheme 5.1. Decomposition of 2-methylbutyraldehyde to generate acrolein and propionaldehyde 108 


\section{CHAPTER 1}

\section{DETECTION AND ANALYSIS OF VOLATILE ORGANIC COMPOUNDS}

1.1. HYPOTHESIS AND CONTRIBUTION OF THIS DISSERTATION

1.2. INTRODUCTION

1.3. PRECONCENTRATION TECHNIQUES

1.4. SURVEY OF REAGENTS FOR DERIVATIZATION OF CARBONYL COMPOUNDS

1.5. ANALYTICAL TECHNIQUES

1.6 DISSARTATION ORGANIZATION 


\subsection{HYPOTHESIS AND CONTRIBUTION OF THIS DISSERTATION}

Because many of available carbonyl-selective reagents including 2,4dinitrophenylhydrazine $(2,4-\mathrm{DNP})^{1-2}$ do not react efficiently with $\alpha, \beta$-unsaturated carbonyl compounds (many of which have been identified either as causative agents or promising markers of diseases), there is a need to develop more effective derivatization reagents for analysis of $\alpha, \beta$-unsaturated carbonyl compounds. Aminooxy and hydrazine moieties are more reactive toward aldehydes and ketones than standard amines due principally to the $\alpha$-effect. ${ }^{3-4}$ The $\alpha$-effect refers to high nucleophilicity induced by the presence of a lone electron pair on an atom immediately adjacent (i.e., "alpha") to the nucleophilic atom (scheme 1.0). Consequently, aminooxy- and hydrazine-based reagents have been used widely to chemoselectively react with carbonyl compounds

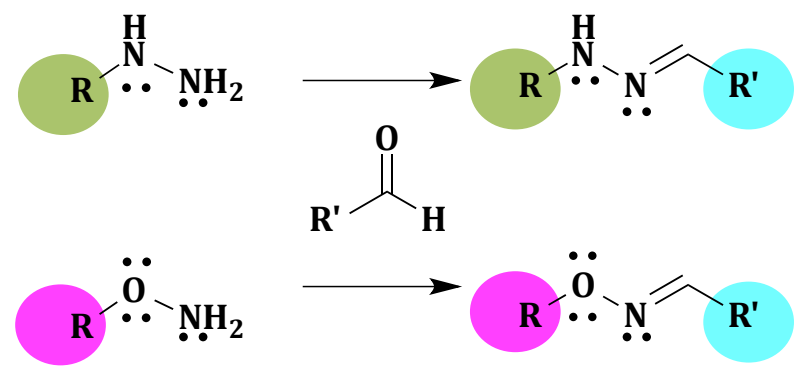

Scheme 1.0. Formation of hydrazones and oximes by reaction of alpha nucleophiles with carbonyl compounds

The condensation reactions of 2-(aminooxy) ethyl-N, N, N-trimethylammonium iodide (ATM) however, with $\alpha, \beta$-unsaturated carbonyl compounds, are notably less efficient so much so that we sought to employ analogous reagent 2-hydrazinyl- $\mathrm{N}, \mathrm{N}, \mathrm{N}$ trimethylethan-1-ammonium iodide (HTM, Scheme 1.1) as a means to improve the capture efficiency for this class of compounds. Since oxygen is more electronegative than nitrogen, hydrazine is expected to be more nucleophilic than aminooxy and should have 
higher reactivity towards carbonyl compounds, especially unsaturated species. We therefore proposed investigating 2-hydrazinyl- $N, N, \quad N$-trimethylethan-1-ammonium iodide abbreviated HTM (Figure 1.0).

We also sought the utility of 2-(aminooxy) ethyl-N, N, N-trimethylammonium iodide (ATM) and 4-(2-aminooxyethyl)-morpholin-4-ium chloride (AMAH) as carbonyl capture reagents for electronic cigarette aerosol analysis (Figure 1.0). While the hydrazine and aminooxy functionalities will enable chemoselective derivatization of carbonyl compounds in the analytical samples, the quaternary ammonium functional group, a permanent positive charge on ATM and HTM, and a titratable positive charge on AMAH, enables a microchip preconcentration approach (through electrostatic bonding with micropillars of the microreactor) of the volatile organic compounds in electronic cigarettes, tobacco cigarettes and exhaled breath. The quaternary ammonium functional group also improved the ionization efficiency of the adduct in direct infusion electrospray ionization. $^{5}$

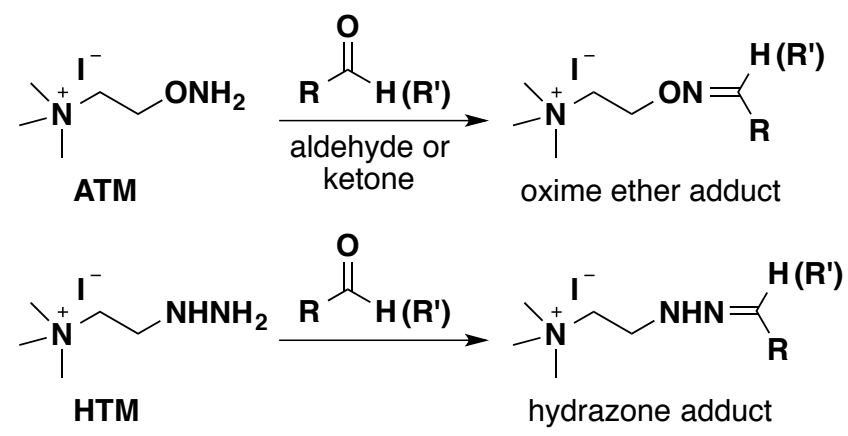

Scheme 1.1. Condensation reactions of aminooxy reagent ATM and hydrazine reagent HTM with aldehydes and ketones.

The pyrolysis of propylene glycol and glycerol generates carbonyl compounds. Since an electronic liquid humectant is principally propylene glycol or glycerol or a 
mixture of both, with our aminooxy or hydrazine-coated, silicon-based microreactor, we could capture and derivatize the carbonyl compounds emitted during aerosolization of eliquids. We were therefore motivated to determine the contribution of the propylene glycol and glycerol to the generation of carbonyl compounds and to both detect and accurately measure aldehydes from aerosols of e-cigarettes using our silicon-based microreactor

This project also sought to quantify nicotine in e-cig liquids and derived aerosol. Direct protonation of pyrrolidine nitrogen of nicotine with an organic acid afforded quaternary ammonium functional group (Figure 1.0) which would also enhanced the ionization efficiency of the nicotinium chloride by FT-ICR-MS.

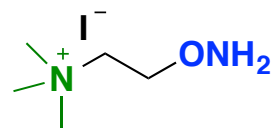

ATM

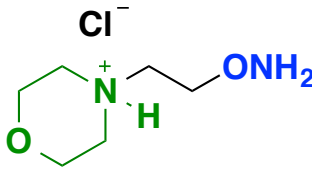

AMAH

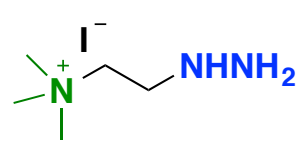

HTM

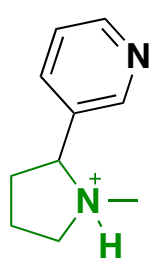

Nicotinium salt

Figure 1.0. The structures of ATM, AMAH, and HTM showing aminooxy and hydrazine functional groups to chemoselectively react with aldehydes and ketones. The positive end as well as that of the nicotinium salt will enhance electrospray ionization efficiency.

\subsection{INTRODUCTION}

Detection and analysis of trace carbonyl compounds, especially aldehydes and ketones, are of high importance. Some of these carbonyls especially acrolein, acetaldehyde, and formaldehyde, are considered the most significant cardiovascular and pulmonary toxins in tobacco smoke. ${ }^{6}$ For instance, many studies have shown that acute exposure to low levels of acrolein can induce dyslipidemia, ${ }^{7}$ vascular injury, ${ }^{8}$ endothelial 
dysfunction, ${ }^{9}$ and platelet activation, ${ }^{10}$ whereas chronic exposures accelerate cardiovascular disease (CVD). ${ }^{11-15}$

Carbonyl compounds can be found in the atmosphere. A trace amount of formaldehyde, derived from photochemical oxidation (hydroxyl radicals) of hydrocarbons, is present in the air. ${ }^{16-17}$ Automobile exhaust in urban centers are a significant source of important aldehydes in air both via direct emission of aldehydes and via emission of hydrocarbons, which in turn are converted to aldehydes through photochemical oxidation reactions. Because of the rise in the use of alternate and reformulated fuels, the number of carbonyl compounds has increased. Increased amounts of formaldehyde or acetaldehyde are now emitted in automobile exhaust depending on the type of oxygenated additives (ethanol, methanol, or methyl tert -butyl ether) added to the automotive fuels. ${ }^{18-20}$ Using Los Angeles as an example, The most abundant carbonyls expressed as percentages of Los Angeles air carbonyl content on a parts per billion basis are formaldehyde (24\%), acetaldehyde (18\%), glyoxal/methylglyoxal (8\%), acetone $(7 \%)$, and acrolein $(5 \%) .^{21-22}$

Also, a large number of toxic compounds have been reported in cigarette smoke, including over 3000 organic chemicals. ${ }^{23}$ These toxic compounds also include polynuclear aromatic hydrocarbons, N-nitrosamines ${ }^{24-25}$, dioxins ${ }^{26}$ acrylamide $^{27}$ and very importantly reactive carbonyl compounds such as acrolein, glyoxal, methylglyoxal, and malonaldehyde (MA). ${ }^{28}$ These toxic aldehydes in cigarette smoke are of great importance because tobacco smoke is one of the major sources of toxic aldehydes contamination in indoor air. 
Besides, carbonyl compounds have been used as chemical markers for disease screening and diagnosis. ${ }^{29,30-31}$ For example, the detection of 4-hydroxyhexenal (4HHE) and 4-hydroxy-2-nonenal (HNE) in expired breath has been correlated with lung cancer. ${ }^{32,33}$ Lipoperoxidative production of reactive aldehyde species, such as malondialdehyde (MDA), HNE, 4-hydroxy-2-hexenal (HHE), and acrolein, appears to be the source of many of the $\alpha, \beta$-unsaturated carbonyl compound markers associated with disease. $^{34}$

The VOCs concentrations are typically low in some of the analytical samples. These concentrations range from a few parts per million ( $\mathrm{ppm}$ ) to a few parts per trillion (ppt). ${ }^{35-36}$ Therefore, preconcentration of VOCs is necessary before analysis.

\subsection{PRECONCENTRATION TECHNIQUES}

Various preconcentration methods for detection and analysis of carbonyl compounds are now available. These techniques can be used for carbonyl compounds in tobacco cigarette, electronic cigarette and exhaled breath analysis. Among them are physical adsorption, solid phase microextraction, and microelectromechanical systems (MEMS) preconcentration devices. For example, analyte adsorptions and subsequent desorption and determination using gas- chromatography mass spectrometry (GC/MS) is a popular preconcentration approach. Adsorbents in the collection traps must be carefully selected to prevent carryover and breakthrough effects. Activated charcoal, different types of graphitized carbon, molecular sieves, and organic polymers have been used for enrichment of VOCs in analytical samples. 


\subsubsection{Physical adsorption}

Table 1.0 shows different adsorbent materials and their composition. The sorbents used for the adsorptive enrichment in combination with thermal desorption should generally meet the following conditions to ensure accurate determination of VOCs:

1. Total enrichment of target analytes

2. Total and fast desorption of analytes

3. The surface must be inert and homogeneous to prevent artifact formation, irreversible desorption and catalytic result during sampling, storage of the loaded adsorbent tubes and desorption

4. Low adsorption capacity for other inorganic constituents of air, for example, nitrogen oxide, sulfur dioxide, carbon dioxide or ozone

5. High thermal and mechanical stability

6. Unreactive towards species like ozone

7. Lack of affinity to water to prevent displacement and hydrolysis and to reduce interferences with gas chromatographic analysis such retention time shift

8. Multiple usability. 
Table 1.0. Adsorbent materials and their composition. ${ }^{37}$

\begin{tabular}{|c|c|c|c|c|c|c|c|c|c|c|}
\hline \multirow[t]{2}{*}{ Adsorbent } & \multirow{2}{*}{$\begin{array}{l}\text { Sampling } \\
\text { range }\end{array}$} & \multirow{2}{*}{$\begin{array}{l}\text { T Max } \\
\left({ }^{\circ} \mathrm{C}\right)\end{array}$} & \multicolumn{6}{|c|}{ Elemental Composition } & \multirow{2}{*}{$\begin{array}{l}\text { Density } \\
\left(\mathrm{g} \mathrm{mL}^{-1}\right)\end{array}$} & \multirow{2}{*}{$\begin{array}{l}\text { Specific } \\
\text { surface } \\
\text { area } \\
\left(\mathrm{m}^{2} \mathrm{~g}^{-1}\right)\end{array}$} \\
\hline & & & $\mathrm{C}$ & $\mathrm{H}$ & $\mathrm{N}$ & S & 0 & $\mathrm{Cl}$ & & \\
\hline \multicolumn{11}{|c|}{ Carbon molecular sieves } \\
\hline Carboxen 563 & $\mathrm{C} 2-\mathrm{C} 5$ & $>400$ & 81.4 & 2.6 & - & 10.6 & 4.7 & 0.1 & 0.53 & 510 \\
\hline Carboxen 564 & $\mathrm{C} 2-\mathrm{C} 5$ & $>400$ & 88.7 & 0.8 & - & 5.8 & 2.4 & 0.6 & 0.60 & 400 \\
\hline Carboxen 569 & $\mathrm{C} 2-\mathrm{C} 5$ & $>400$ & 93.7 & 0.3 & - & 4.0 & 1.1 & 0.4 & 0.58 & 485 \\
\hline Carboxen 1000 & $\mathrm{C} 2-\mathrm{C} 5$ & $>400$ & 91.9 & 0.9 & - & 0.2 & 4.0 & 1.3 & 0.44 & 1200 \\
\hline Carboxen 1001 & $\mathrm{C} 2-\mathrm{C} 5$ & $>400$ & 93.2 & 0.6 & - & 3.3 & 1.3 & 1.2 & 0.61 & 500 \\
\hline Carboxen 1003 & $\mathrm{C} 2-\mathrm{C} 5$ & $>400$ & 95.8 & 0.28 & - & 2.41 & 0.9 & 0.6 & 0.46 & 1000 \\
\hline Carbosieve SIII & $\mathrm{C} 2-\mathrm{C} 5$ & $>400$ & 95.7 & 0.8 & - & - & 2.0 & 1.1 & 0.61 & 820 \\
\hline Carbosphere & - & 400 & 90.5 & 0.5 & - & 0.1 & 2.1 & 0.2 & - & 1000 \\
\hline \multicolumn{11}{|c|}{ Graphitized carbon blacks } \\
\hline Carbotrap F & $>\mathrm{C} 20$ & $>400$ & 99.8 & 0.1 & - & - & 0.1 & 0.1 & 0.66 & 5 \\
\hline Carbotrap C & C12-C 20 & $>400$ & 99.7 & 0.1 & - & - & - & 0.2 & 0.72 & 10 \\
\hline Carbotrap GCB & C5-C12 & $>400$ & 99.9 & 0.1 & - & - & - & - & 0.36 & 100 \\
\hline Carbotrap Y GCB & $\mathrm{C} 12-\mathrm{C} 20$ & $>400$ & 99.6 & 0.2 & - & - & - & 0.3 & 0.42 & 25 \\
\hline Carbotrap X GCB & C3-C5 & $>400$ & 99.2 & 0.1 & - & 0.1 & - & 0.1 & 0.41 & 250 \\
\hline Carbograph 5 GCB & C3-C5 & $>400$ & 100 & - & - & - & $-\quad-$ & - & - & 560 \\
\hline \multicolumn{11}{|c|}{ Porous organic polymers } \\
\hline $\begin{array}{l}\text { Chromosorb106 } \\
\text { (Styrene-divinyl- } \\
\text { benzene-copolymer) }\end{array}$ & Small molecule & 250 & 92.1 & 7.8 & - & 0.2 & 0.7 & 0.3 & - & 750 \\
\hline
\end{tabular}

\subsubsection{Solid phase microextraction}

The most laborious and time-consuming parts of chemical analysis are sample preconcentration and preparation. Currently sample preparation methods have received great attention. The requirement for their miniaturization, avoidance of toxic solvents, and the ease of coupling with analytical methods have been put to the fore. ${ }^{38}$ Solid Phase Microextraction (SPME) was developed by by Arthur and Pawliszyn in the early 90s as a rapid extraction and preconcentration method for analyzing volatile and semi-volatile compounds from a variety of matrices. ${ }^{39}$ It integrates sampling, extraction, concentration and sample introduction into a single solvent-free step. Analytes in the sample are 
directly extracted and concentrated on an extraction fiber. ${ }^{40}$ Its advantages include miniaturization ability; reduction of sample preparation time; reduction or even complete elimination of toxic solvents; high concentration factors; low cost; simplicity of coupling with instrumental methods of analysis; and possibility of automation. ${ }^{41}$

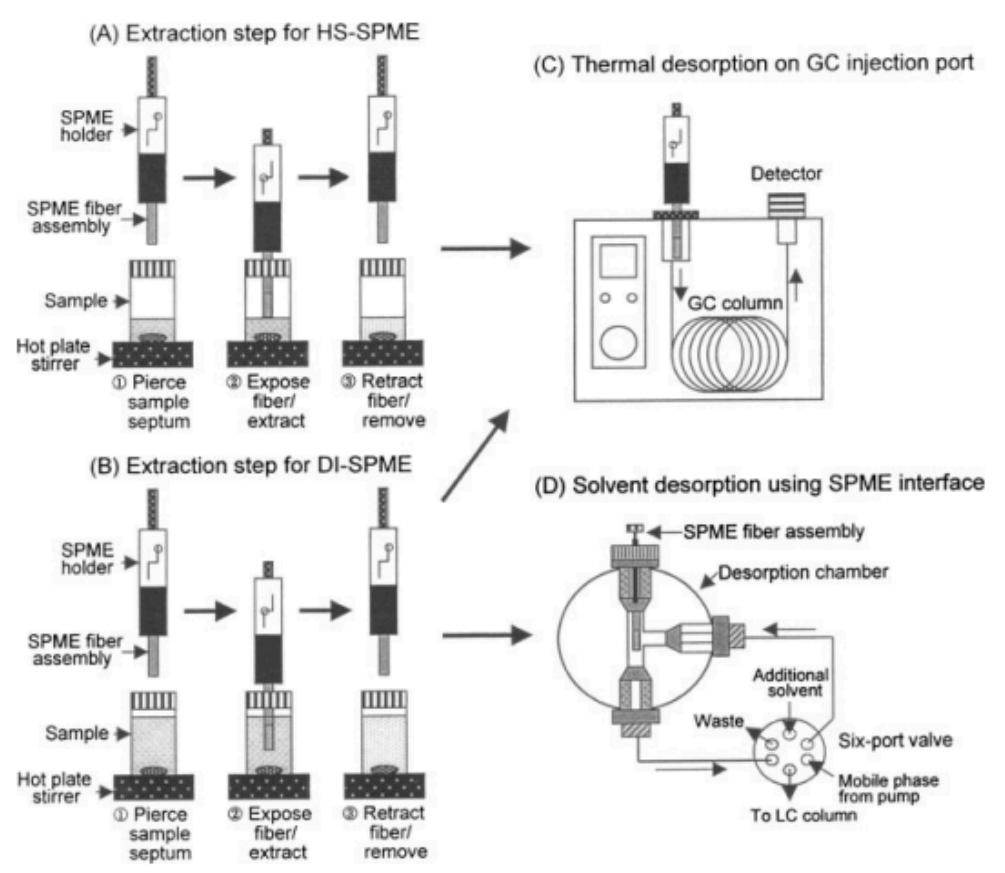

Figure 1.1. SPME procedure for GC and LC. Reproduced from Vas, G.; Vékey, K., J. Mass Spectrom. 2004; 39:233-254 Solid-phase microextraction: a powerful sample preparation tool prior to mass spectrometric analysis. ${ }^{40}$

SPEME coatings are made with organic and organosilicon polymers or hybrid organomineral adsorbents. The most commonly used materials for preconcentrating nonpolar and weakly polar compounds are polymeric coatings of polydimethylsiloxane (PDMS), polyacrylate (PAC), polydivinylbenzene (DVB). ${ }^{42}$ The sampling process is illustrated in Figure 1.0. The sample is placed in a vial and sealed with a Mininert valve. Partitioning between the sample matrix and the stationary phase takes place when the SPME needle pierces the septum and the fiber is extended through the needle into the 
sample. If the fiber is exposed to only vapor phase above a gaseous, liquid or solid sample, the process is headspace, (HS-SPME). On the other hand, if the fiber is directly immersed in liquid samples, then the process is direct-immersion, (DI-SPME). After extraction, the fiber is withdrawn back into the needle assembly and removed from the valve and inserted immediately into the GC injection port. SPME can be used with GC, GC-MS HPLC and HPLC-MS..$^{40,43-44}$

\subsubsection{Microfluidic chip}

The use of microfluidic system as preconcentration devices has attracted considerable attention and has experienced rapid growth in the past two decades because of the promise of miniaturization, real-time analysis and power efficiency, and integration and automation. ${ }^{45}$ A typical preconcentration device fabricated on a silicon wafer by Alfeeli and Agah ${ }^{46}$ consists of embedded high aspect-ratio three-dimensional (3-D) micro pillars coated with an adsorbent polymer. Their work was applied to handheld point-of-care breath analysis instrumentation by preconcentrating VOCs, like ndecane $\left(\mathrm{C}_{10}\right)$, n-dodecane $\left(\mathrm{C}_{12}\right)$, 2,6-diisopropylphenol (Propofol), n-tetradecane $\left(\mathrm{C}_{14}\right)$, and $\mathrm{n}$-hexadecane $\left(\mathrm{C}_{16}\right)$, in exhaled breath of patients undergoing anesthesia. Fu et al ${ }^{47}$ also fabricated a silicon-based microreactor containing thousands of micropillars in channels for capture of trace-level volatile aldehydes and ketones. By chemically functionalizing each micropillar surface, gaseous aldehydes and ketones in exhaled breath samples are chemoselectively preconcentrated thereby enabling ultra-trace, rapid analysis by direct-infusion Fourier transform-ion cyclotron resonance (FT-ICR) mass spectrometry (MS). The micropillar surfaces ((Figure 1.2) are coated with quaternary 
ammonium aminooxy salt 2-(aminooxy) ethyl-N, N, N-trimethylammonium iodide (ATM) (Figure 1.1) for capturing trace carbonyl VOCs by means of an oximation reaction. This silicon-based microreactor system was used for sample preconcentrations for the purpose of this dissertation.

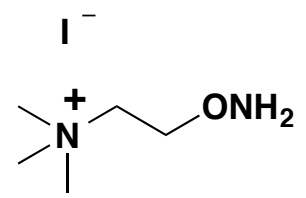

Figure 1.2. 2-(aminooxy) ethyl-N, N, N-trimethylammonium iodide (ATM)

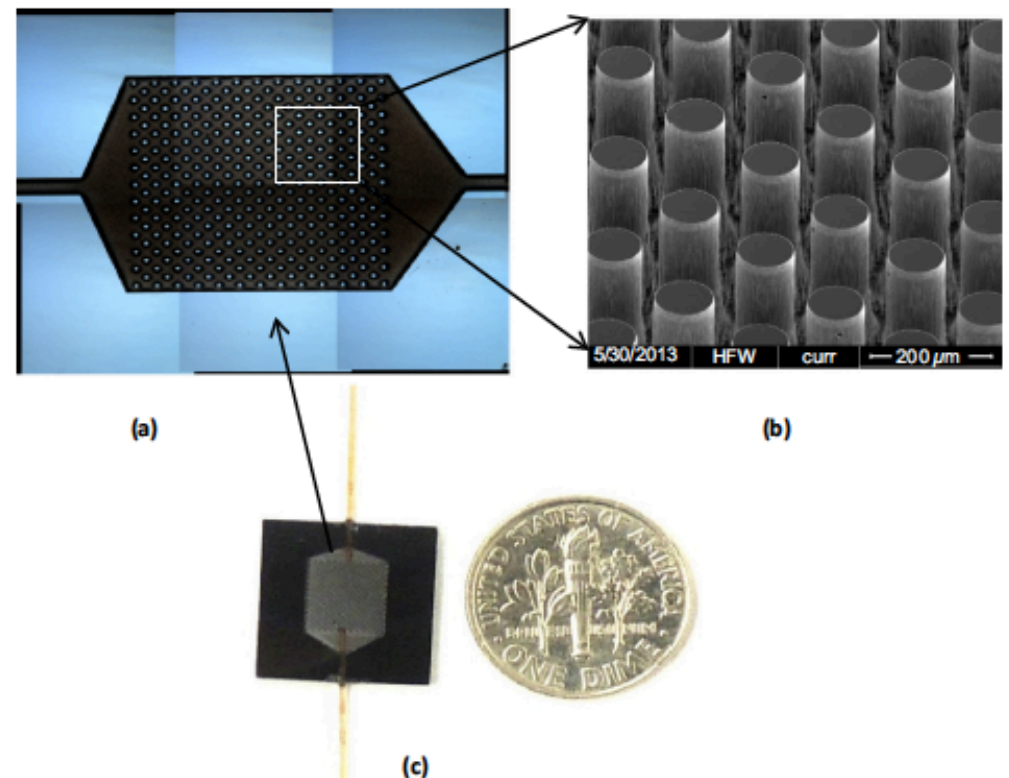

Figure 1.3. Photographs of the microreactor: (a) Optical micrograph of the microreactor before bonded with a glass wafer. (b) SEM micrograph of the micropillar array in the microreactor. (c) The microreactor connected to two fused silica tubes. A dime was placed near the microreactor to indicate the size of the microreactor, (Photo adapted from $\mathrm{Fu}$ et $a l .{ }^{31}$ ). 


\subsection{SURVEY OF DERIVATIZATION REAGENTS FOR CARBONYL COMPOUNDS}

The analysis and quantification of low molecular weight aldehydes and ketones is particularly difficult because many of the carbonyl compounds are volatile, unstable, reactive and have low abundance. ${ }^{48}$ The conventional way to mitigate this problem is to derivatize these carbonyl compounds with a suitable reagent. Although the number of derivatization reagents for aldehydes and ketones have increased considerably over the last decade, a majority of these derivatization reagents are not efficient in derivatizing unsaturated carbonyl compounds. Among the existing derivatization reagents for aldehyde and ketones are aryl hydrazines, such as 2,4-dinitrophenylhydrazine (2,4DNP). ${ }^{1,2,49,50,51}$ Other reagents include 4-hydrazino-4-oxobutyl-[tris $(2,4,6-$ trimethoxyphenyl)- phosphonium bromide (TMPP-PrG), 1-(Carboxymethyl)pyridinium chloride hydrazide (Girard's reagent P), (Carboxymethyl)trimethylammonium chloride hydrazide, (Girard's reagent T), and pentafluorophenylhydrazine $(\mathrm{PFH})^{52-53}$ and the newly developed aminooxy-based 2-(aminooxy)- $N, N, N$-trimethylethan-1-ammonium iodide $(\mathrm{ATM})^{54,31,47}$ and 4-(2-(aminooxy)ethyl)-morpholin-4-ium chloride (AMAH) ${ }^{55}$ reagents (Figure 1.3). A typical derivatization reaction of carbonyl compounds with Girard's reagent $\mathrm{T}$ leads to the formation of an acyl hydrazone while AMAH will undergo the same reaction to form an oxime ether as shown in scheme $1.2 .^{56}$

The derivatives of the carbonyl compounds are usually analyzed with a suitable analytical detection instrument. For the purpose of this study, cationic aminooxy and hydrazine -based reagents were used because of their high reactivity and easy adaptability to FT-ICR-MS. 


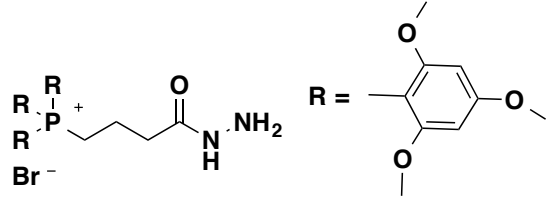

TMPP-PrG

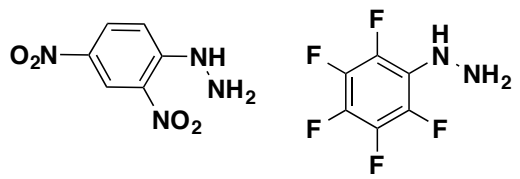

DNPH

PFH<smiles>NNC(=O)CN1C=CC=CC1</smiles>

Girard's Reagent P

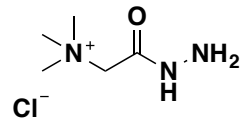

Girard's Reagent T<smiles>C[N+](C)(C)CCON</smiles>

ATM<smiles>NOCCN1CCOCC1</smiles>

AMAH

Figure 1.4. Reagents for derivatization of carbonyl compounds.<smiles>C[N+](C)(C)CC(=O)NN</smiles>

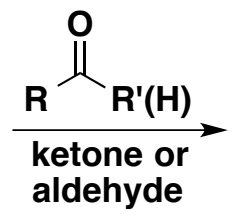<smiles>[R7]C([R17])=NNC(=O)C[N+](C)(C)Cl</smiles><smiles></smiles>

AMAH

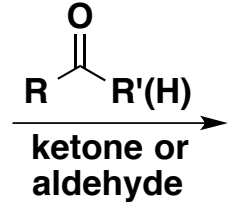

aldehyde<smiles></smiles>

AMAH adduct

Scheme 1.2. Derivatization of carbonyl compounds with Girard's reagent $\mathrm{T}$ and AMAH.

Volatile carbonyl compounds are conventionally derivatized with 2,4dinitrophenylhydrazine (DNPH). The DNPH are usually acidified with a mineral acid like hydrochloric acid and impregnated on silica gel. ${ }^{23,57}$ They are sold in pre-packed cartridges. After derivatization, the DNPH-carbonyl adducts are washed off from the cartridge with acetonitrile and an aliquot of the adducts are analyzed and quantified with HPLC (Figure 1.4). DNPH can also be acidified and used in an impinger trap containing 
a known amount of acetonitrile to trap and derivatize the carbonyl compounds followed by analysis with HPLC. These techniques usually involve the use of an acid to drive the derivatization reaction. They also are cumbersome, involve the use of large volume of solvents during HPLC analysis and have fewer prospects for miniaturization. Besides, the DNPH cartridge is usually not re-useable.

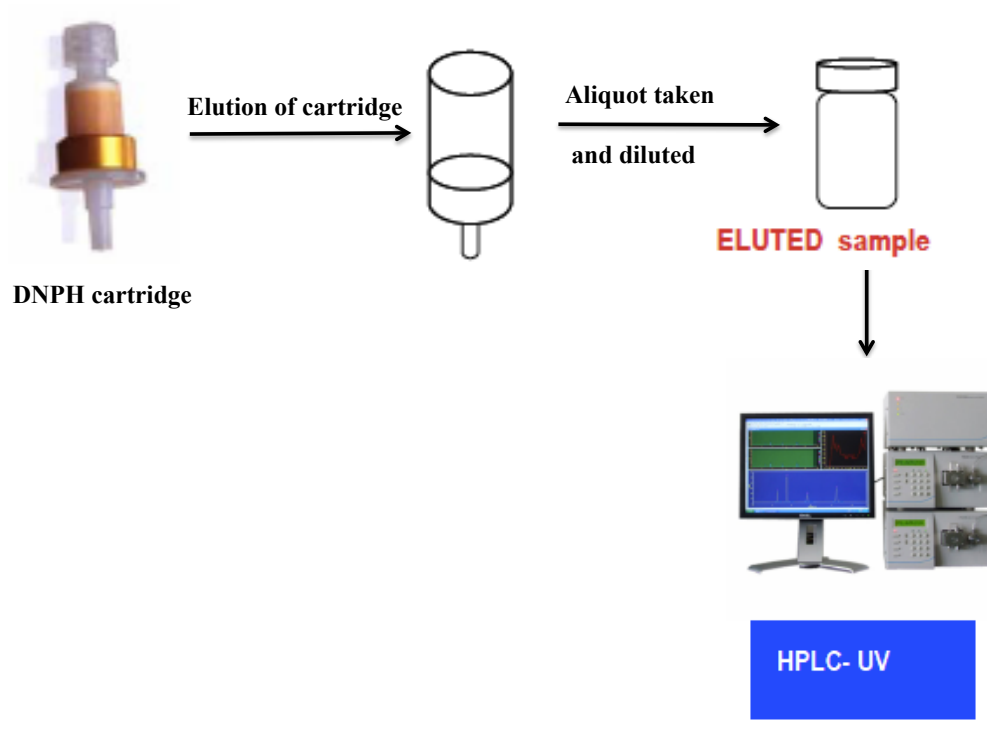

Figure 1.5. DNPH cartridge for derivatization of carbonyl compounds.

\subsection{ANALYTICAL DETECTION TECHNIQUES}

Conventional spectroscopic methods such as infrared, fluorescent and nuclear magnetic resonance spectroscopic methods have been employed in the analysis of VOCs, especially carbonyl compounds. However these methods suffer from inadequate specificity, sensitivity, and inability to detect many classes of compounds. ${ }^{58}$ Thus, there is a need for analytical methods that can provide high sensitivity, specificity, and adequate mass resolving power. Gas chromatography mass spectrometry (GC-MS), liquid chromatography-tandem mass spectrometry (LC-MS/MS), ion mobility mass 
spectrometer (IMS), electronic nose, and fourier transform ion cyclotron resonance mass spectrometry (FT-ICR-MS) are instrument of choice for a analysis of VOCs.

\subsubsection{Gas chromatography mass spectrometry}

Gas chromatography coupled with mass spectrometry (GC-MS) is the most commonly used analytical technique for trace-level VOCs due to its high sensitivity and reliability in analyte identification. Also, GC-MS has been used for identification and analysis of certain metabolic products like acetone, ethanol and isoprene, and other VOCs in normal human expired breath. It has also been used for analyzing carbonyl compounds in electronic cigarette aerosols. ${ }^{59}$ More detailed analytical information and analyte identity can be derived from GC- MS than the proton transfer reaction mass spectrometry (PTR-MS). Multidimensional gas chromatography introduced few decades ago, especially two-dimensional gc typically coupled with a time-of-flight or quadrupole mass analyzer mass spectrometer, has also been used to analyze volatomes in exhaled breath to provide a more effective way of enhancing the resolving power. ${ }^{60-61}$ Even though the use of GC-MS for analysis of preconcentrated VOCs is both sensitive and reliable, and relatively cheap, it has the downside of laborious sample preparation and the whole process is cumbersome.

\subsubsection{Liquid chromatography-tandem mass spectrometry (LC-MS/MS)}

Liquid Chromatography coupled with tandem mass spectrometry is a powerful technique for analysis of biological samples without extensive sample preparation. It has

also found application in the analysis of carbonyl compounds in e-cigarette aerosols. ${ }^{62}$ 
Accuracy and precision at very low analyte concentrations however are a challenge when using this technique.

\subsubsection{Ion mobility mass spectrometer (IMS)}

Another analytical detection technique is ion mobility spectrometry (IMS). It is not yet very popular in the study of VOCs of cancer markers. Westhoff $e t a l^{63}$ carried out the first study of VOCs with IMS. IMS has low selectivity hence complex mixtures are usually analyzed with a pre-preparation method like multi-capillary column (MCC). It is however a comparatively cheap detection technique with high promise for miniaturization. ${ }^{64}$

\subsubsection{Electronic nose}

An electronic nose contains an array of nonspecific, gas sensitive, chemical sensors as artificial odor receptors. An electronic nose sensor includes components such as a quartz microbalance, carbon-polymer array, and colorimetric sensor array ${ }^{65-66}$ In an electronic nose (E-Nose), the VOCs adsorb onto a sensor where a change in conductivity, color or oscillation of a crystal is detected by the sensing system of the E-nose. The Enose responds to only a mixture of compounds in the sample. The pattern recognition system (computer) interprets and detects the high levels of VOCs. The drawbacks include the extensive preparation of the breath samples, lack of quantitative data and calibration. ${ }^{67}$ 


\subsubsection{Fourier transform ion cyclotron resonance mass spectrometry (FT-ICR-MS)}

The advent of ultrahigh resolution mass spectrometers has created opportunities for exploration of high-throughput analysis of trace volatile carbonyl compounds in breath, electronic cigarettes and environment, especially in conjugation with novel chemoselective (CS) probes designed for these instruments.

Fourier transform ion cyclotron resonance mass spectrometry (FT-ICR-MS) can analyze several compounds in seconds via extreme mass resolution combined with ultrahigh mass accuracy mass-resolving power. ${ }^{68-69}$ The resolution of FT-ICR-MS is very high that it can fit several of unique chemical ions, including all stable isotopes, in a single analysis, eliminating the time-consuming chromatography that has plagued analysis of highly complex mixtures by all other types of MS. These characteristics make it particularly suitable for analysis of volatile aldehydes and ketones in electronic cigarette aerosols and exhaled breath. FT-ICR-MS was therefore an analytical detection instrument of choice for my graduate research.

With the use of a silicon-based microreactor on which a highly reactive aminooxy or a hydrazine reagent is coated as a preconcentration method of choice and high resolution FT-ICR-MS as analytical detection tool, the problems associated traditional DNPH-silica gel cartridge method, including cumbersomeness, the use of large volume of solvent nonresusability, and lack of prospect for miniaturization can be avoided.

However, despite the advantages offered by FT-ICR-MS, it is difficult to distinguish between two compounds having the same mass to charge ratio in full scan mode except by tender mass spectrometry (MS/MS) and quantification of such compounds when they were present became difficult. ${ }^{70}$ Because of difficulties 
experienced with the MS/MS we therefore turned to gas chromatography mass (GC-MS) spectrometry to separate functional isomers like acetone and propanal, butanone and butanal.

\subsection{DISSERTATION ORGANIZATION}

This dissertation contains five chapters. Chapter II presents the results of studies using the existing 4-(2-aminooxyethyl)-morpholin-4-ium chloride (AMAH) derivatization reagent in the analysis of carbonyl compounds in electronic cigarette aerosols using the prefabricated microreactor technology enabled with gas chromatography-mass spectrometry (GC-MS) and Fourier Transform Ion Cyclotron Mass Spectrometry FT-ICR-MS.

Chapter III describes a kinetics study on the oximation reactions of selected aminooxy and carbonyl compounds. It also shows the synthesis, characterization and utility of novel reagent 2-hydrazinyl- $N, N, N$-trimethylethan-1-ammonium iodide (HTM) in analysis of carbonyl compounds, especially $\alpha, \beta$-unsaturated species in exhaled breath.

Chapter IV gives a detailed work on the development of a new method for nicotine quantification in electronic cigarettes liquids and aerosols. Chapter V concludes the dissertation and outlines the future work. 


\section{CHAPTER 2}

\section{CARBONYL COMPOUNDS IN ELECTRONIC}

\section{LIQUIDS AND AEROSOLS}

2.1. ELECTRONIC CIGARETTES

2.2. SIGNIFICANCE OF CARBONYLS IN ELECTRONIC CIGARETTES

2.3. E-CIGARETTE AEROSOL GENERATIONS; CAPTURE, DERIVATIZATION AND ANALYSIS OF CARBONYL COMPOUNDS

2.4. RESULTS AND DISCUSSION

2.5 CONCLUSION

2.6 EXPERIMENTAL SECTION 


\subsection{ELECTRONIC CIGARETTES}

Electronic cigarettes are battery-powered, tobacco-free nicotine delivery devices that aerosolize a nicotine-containing solution known as e-liquid or e-juice without combustion or smoke. ${ }^{71-73}$ The electronic cigarette was invented by Chinese pharmacist Hon Lik in the early 2000 s to function as a substitute for quitting smoking. ${ }^{74-76}$ It is designed to look like and provide the feel of a traditional cigarette. ${ }^{77}$ E-juices are usually a mixture of propylene glycol or glycerin, or both, and distilled water laced with nicotine and other food grade additives that may or may not be approved for use by the US Food and Drug Administration (FDA). ${ }^{78}$ The electronic cigarette (e-cigarette) device is electrically driven by a battery -usually a lithium battery -and contains an atomizer in which the e-juice is housed and then aerosolized by application of electrical energy that generates resistance heat encircling a wick (Figure 2.1). ${ }^{79-80}$

Electronic cigarettes come either as disposable or refillable. The disposable unit must be discarded once the electronic liquid is exhausted while the refillable unit, which usually is a tank type, may be used indefinitely because it can be replenished once the liquid is exhausted. ${ }^{81}$ The electronic juice in electronic cigarettes is laced with several flavors, such as chocolate, vanilla, menthol, tobacco or fruit flavors. ${ }^{82}$

E-cigarette usage has surged since being introduced to the US market in 2007 as a safer alternative to traditional cigarettes. There are currently over 40 million smokers in the U.S. and about $20 \%$ of them have tried e-cigarettes. ${ }^{83}$ The growing popularity of ecigarette users has raised serious public health concerns of potential harm to e-cigarette users. ${ }^{15,23,76,84-86}$ The exponential increase of e-cigarette users among young people may also cause nicotine addiction and transition to tobacco cigarettes. ${ }^{76,85}$ One way of 
evaluating the safety of electronic cigarettes as an alternative to traditional cigarettes is by measuring the concentration of carbonyl compounds that can be emitted during vaping.

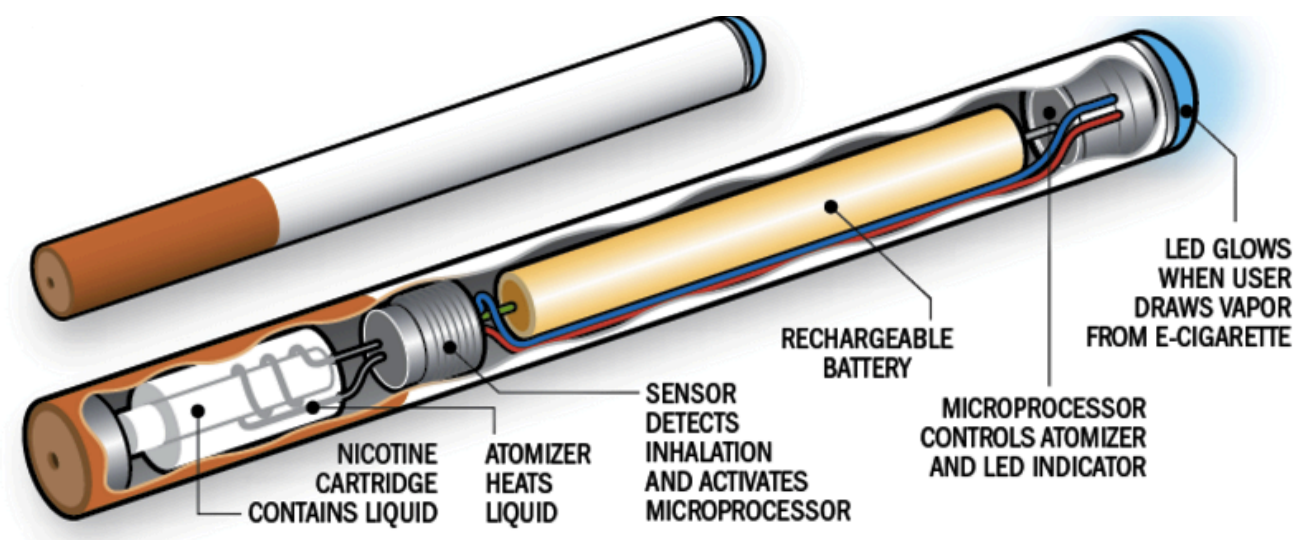

Figure 2.1. Anatomy of an Electronic Cigarette. ${ }^{87}$

\subsection{SIGNIFICANCE OF CARBONLYS IN ELECTRONIC CIGARETTES}

Certain low molecular weight aldehydes like acetaldehyde, acrolein and formaldehyde which are considered very harmful constituents of tobacco smoke ${ }^{88}$ are found in high concentrations in cigarette smoke between 700-800 $\mu \mathrm{g} /$ cigarette in mainstream smoke ${ }^{89-90}$, cigars, and waterpipes (hookah, narghile,), bidis, smokeless tobacco products (snus and snuff), and are also present in e-cigarettes aerosols. ${ }^{91-95}$

A link between e-cigarette use and risk stems from the presence of harmful and potentially harmful constituents identified in aerosols of e-cigs, including metals, particles, carbonyls and flavoring compounds. ${ }^{71,79,96-100}$ However, the presence of toxic carbonyl compounds in e-cigarette aerosols derived from humectants and/or flavoring chemicals used in e-liquid formulations is raising health concerns. ${ }^{98,}{ }^{101}$ In order to 
address these concerns, we set out to measure the generation of carbonyl compounds from the humectants propylene glycol and glycerol, as well as from commercial e-liquids containing flavoring chemicals in "tank type" refillable e-cigarette devices.

The e-cig device, e-liquid composition and puff topography are the principal factors that determine the extent of toxic carbonyl compound generation in the aerosol. The temperature of the heating coil for aerosolization of an e-liquid is a function of the battery power output determined by the battery voltage output and the coil resistance. The temperature of the heating coil is also affected by the amount of heat transferred to vapor or aerosol as determined by the puff volume or air flow rate, puff duration and the puff frequency, and a theoretical model has been developed to predict the coil temperature of the atomizer. ${ }^{102}$ The battery power output is the critical factor for determining the coil temperature, and several publications have indicated that the battery power output dramatically affects generation of aldehydes in aerosols. ${ }^{71,97,103}$ Moreover, high battery power output could cause wick starvation and lead to a "dry puff" wick that exponentially increases the generation of formaldehyde, acetaldehyde and acrolein. ${ }^{71,104}$

Thousands of e-liquids are commercially available. In addition to small amounts of water, nicotine and flavoring compounds, the two main components of e-liquids are humectants, predominately propylene glycol (PG) and vegetable glycerin (VG, glycerol). Our study compared carbonyl compounds generated from varying mixtures of humectants PG and VG with corresponding carbonyl compounds in aerosols of selected popular e-liquids. 


\subsubsection{Pyrolysis of propylene glycol}

Propylene glycol (propane-1, 2-diol, PG) is a hygroscopic organic compound. It is used as pharmaceutical formulation solvent, as a humectant in electronic liquid formulation and in food additives as a plasticizer. It has also found application as a moisturizer in medicine, cosmetics, food, and the tobacco industry.

Propylene glycol has been known to react with $\mathrm{O}_{2}$ between $227-427{ }^{\circ} \mathrm{C}$ using silver (Ag) catalyst to produce acetol (hydroxyacetone), which subsequently undergoes oxidation to form methylglyoxal. ${ }^{105-106}$ The homogeneous oxidation of PG between 127$327{ }^{\circ} \mathrm{C}$ has also been shown to produce acetone, acetaldehyde, formaldehyde, and acetol while acetaldehyde can undergo aldol condensation reaction to form crotonaldehyde (Scheme 2.0).

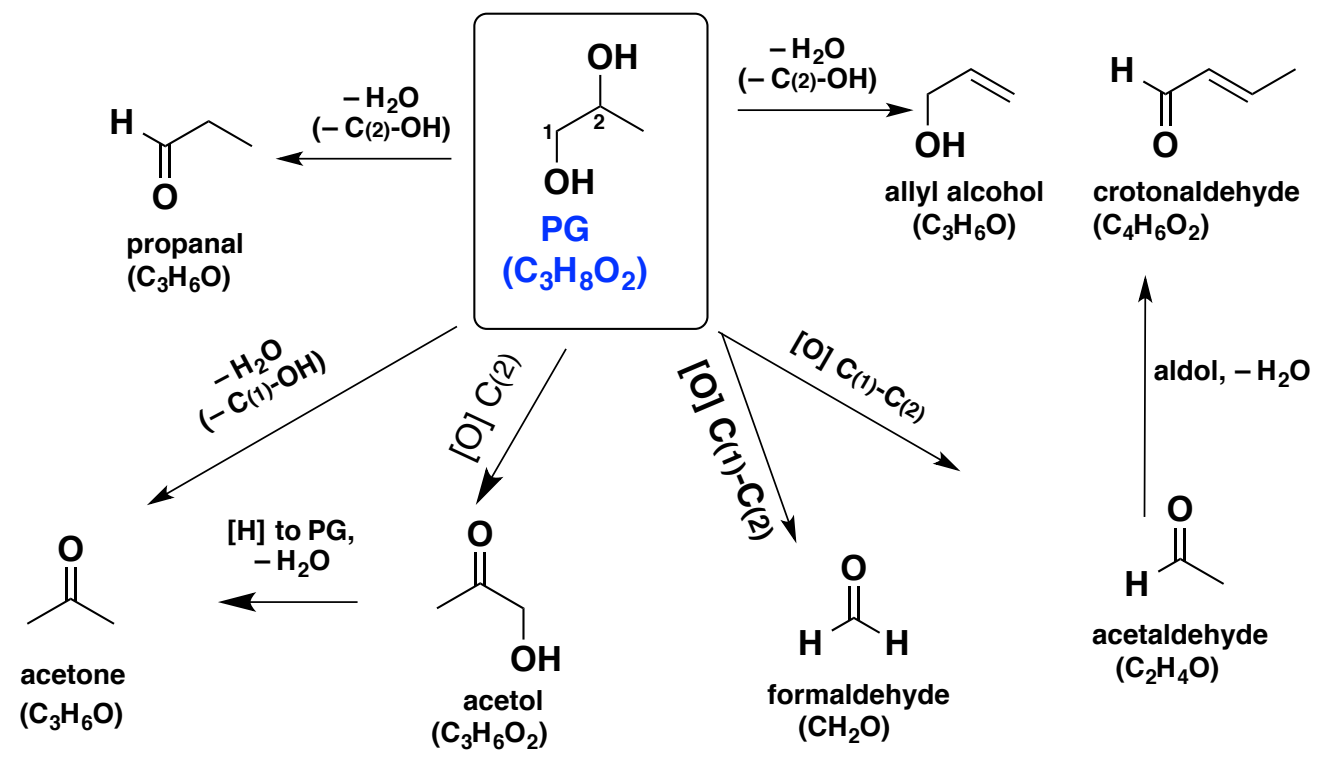

Scheme 2.0. The homogeneous oxidation routes of Propylene glycol as proposed by Diaz et al. ${ }^{107}$ 


\subsubsection{Pyrolysis of glycerol}

The composition of the product mixture formed during the thermal decomposition of glycerol is temperature dependent. For instance, dehydration of glycerol to acrolein is increased at low temperatures as a result of the ionic mechanism and acrolein is obtained as the main product. ${ }^{108}$ The thermal decomposition of glycerol is highly endothermic, and demands a great deal of heat input. This results in steep thermal gradient, and produces various non-equilibrium products. ${ }^{109}$ The two major pathways for thermal decomposition glycerol involve dehydration and dehydrogenation of glycerol. Hydroxyacetone and 3hydroxypropanal are produced during dehydration process (equation 1), the latter being acrolein precursor (equation 2) while glyceraldehydes and dihydroxyacetone are produced during dehydrogenation (equation 3). These compounds can be converted into various intermediates, such as acetaldehyde, and decomposed further into syngas at a high temperature. ${ }^{110}$ Scheme 2.1 shows the possible reactions occurring in glycerol pyrolysis.

$$
\begin{aligned}
& \mathrm{C}_{3} \mathrm{H}_{8} \mathrm{O}_{3} \rightarrow \mathrm{C}_{3} \mathrm{H}_{6} \mathrm{O}_{2}+\mathrm{H}_{2} \mathrm{O} \Delta \mathrm{H}=+450 \mathrm{~kJ} \mathrm{~mol}^{-1} \\
& \mathrm{C}_{3} \mathrm{H}_{6} \mathrm{O}_{2} \rightarrow \mathrm{C}_{3} \mathrm{H}_{4} \mathrm{O}+\mathrm{H}_{2} \mathrm{O} \Delta \mathrm{H}=-36 \mathrm{~kJ} \mathrm{~mol}^{-1} \\
& \mathrm{C}_{3} \mathrm{H}_{8} \mathrm{O}_{3} \rightarrow \mathrm{C}_{3} \mathrm{H}_{6} \mathrm{O}_{3}+\mathrm{H}_{2} \Delta \mathrm{H}=-15 \mathrm{~kJ} \mathrm{~mol}^{-1}
\end{aligned}
$$




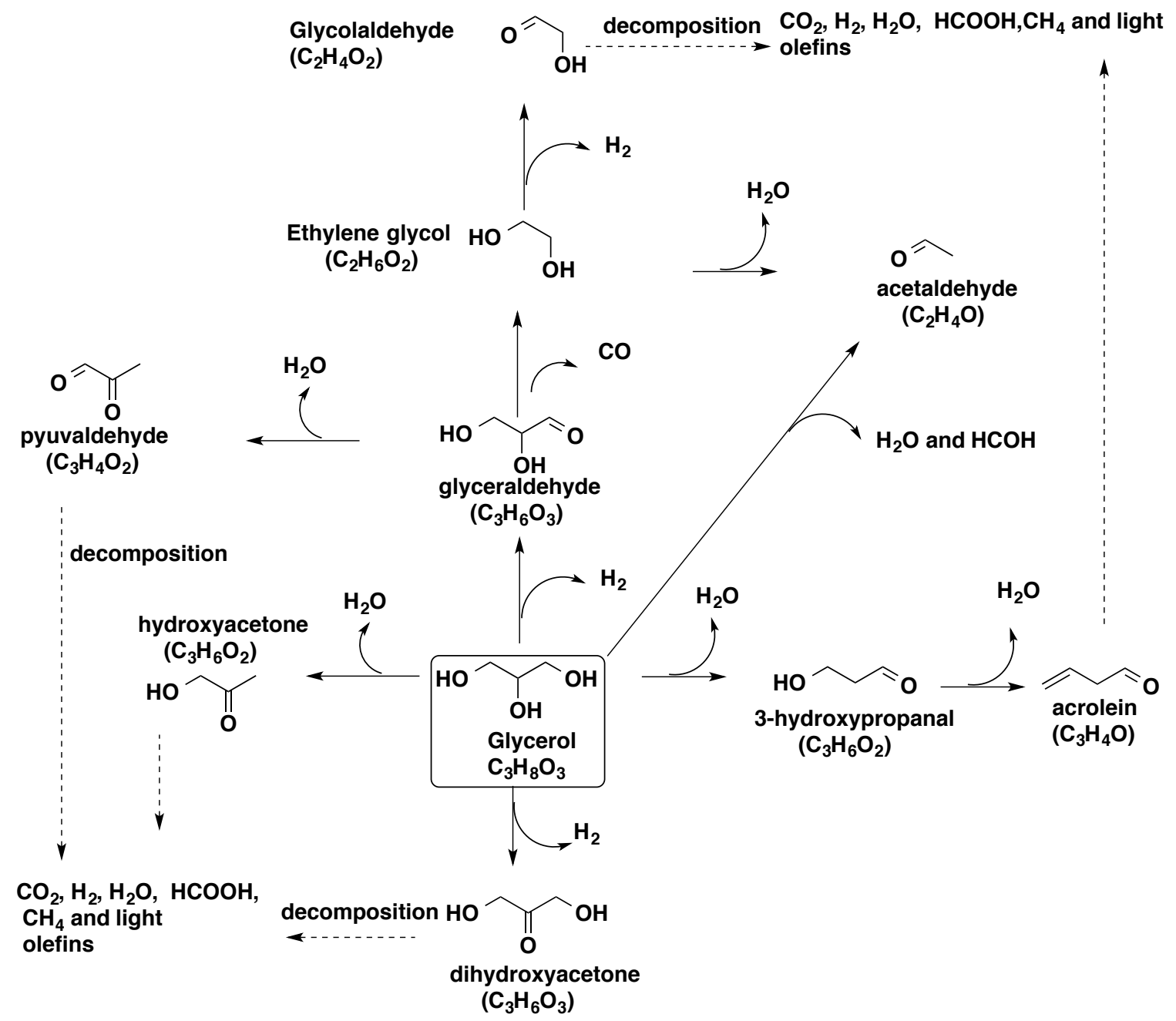

Scheme 2.1. Possible reactions occurring in glycerol pyrolysis (adapted from Yu-Chuan $\left.\operatorname{Lin}^{111}\right)$.

\subsubsection{Hemiacetal formation in aerosols of e-liquids}

Hemiacetals are formed when alcohols, such as propylene glycol and glycerol in e-liquids, add reversibly to the carbonyl functional group of aldehydes ${ }^{12-113}$, as shown in Scheme 2.2. The reaction between formaldehyde and propylene glycol or glycerin of ecigarette liquids during vaporization is therefore thought to form measurable formaldehyde-hemiacetal, as detected by ${ }^{1} \mathrm{H}$ NMR spectroscopy. A recent report suggested that emission of formaldehyde in e-cigarette aerosols is higher than a direct measurement of formaldehyde because a portion of formaldehyde is sequestered in the 
form of a hemiacetal (the so called "hidden formaldehyde"), which prompted more health concerns over using e-cigarettes. ${ }^{71}$ Unfortunately, this illuminating work on measurement of formaldehyde-hemiacetal did not measure free or unreacted formaldehyde or any other aldehydes in the aerosols of the e-cigarettes. We addressed this deficiency by measuring both free formaldehyde as well as formaldehyde-hemiacetal produced during vaping.

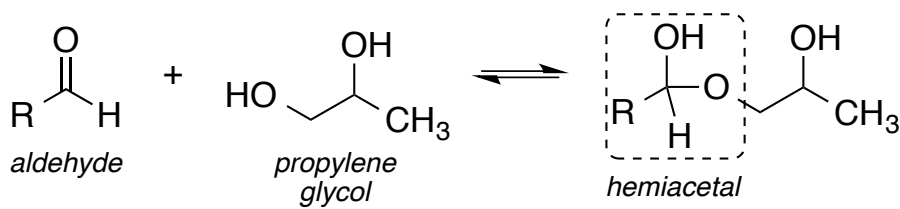

Scheme 2.2. Reversible formation of hemiacetal by reaction of an aldehyde and an alcohol.

\subsection{E-CIGARETTE AEROSOL GENERATION; CAPTURE, DERIVATIZATION AND ANALYSIS OF CARBONYL COMPOUNDS}

In order to accurately quantify the carbonyl compounds in electronic cigarettes aerosols, we use cigarette-smoking robot to puff electronic cigarette and the aerosol samples were collected in a 5 or 10-litre Tedlar bag (Figure 2.2). An aliquot of the aerosol can be transferred to a $1 \mathrm{~L}$ tedlar bag for analysis. We use our powerful silicon microreactor coated with an aminooxy reagent AMAH as a derivatization reagent (coating) reagent and connected to a vacuum pump to pull the aerosols through the microreactor for carbonyl derivatization until the tedlar bag is empty (Figure 2.3). 


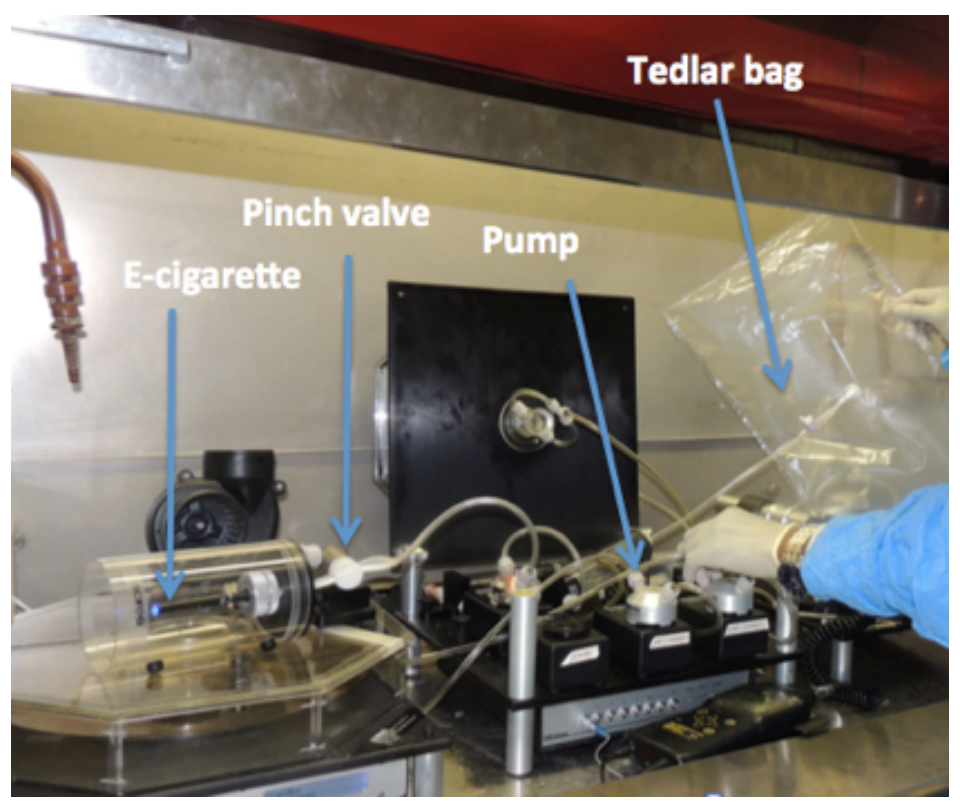

Figure 2.2. Cigarette-smoking robot system for mechanical generation of aerosols from ecigarettes.

After the preconcentration, the microreactor was eluted with methanol followed by the addition of internal standard. The FT-ICR-MS spectra of the samples were obtained and the quantification of carbonyl compounds achieved with the calibration curves.

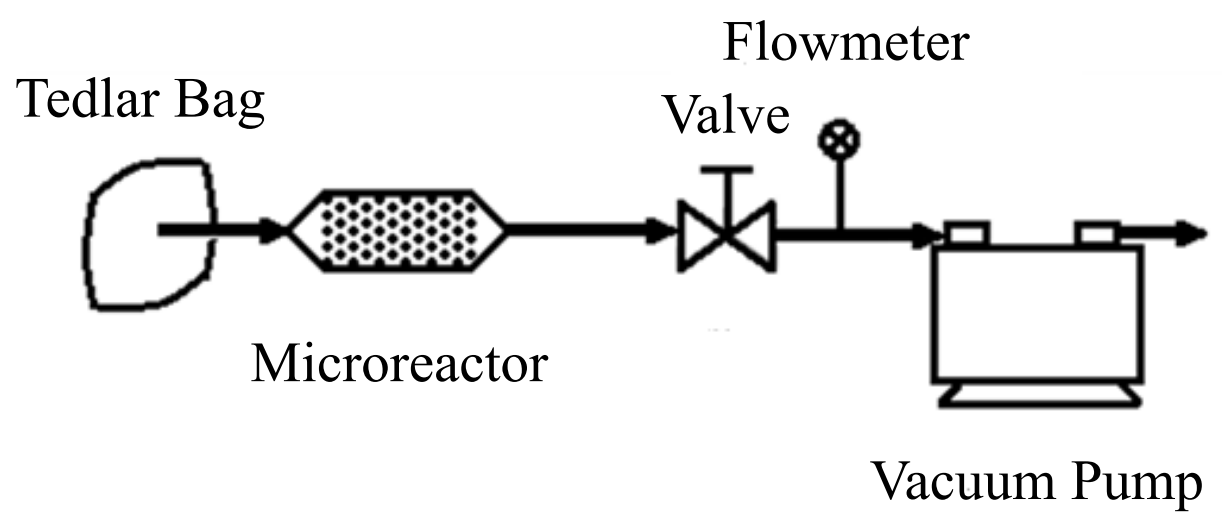

Figure 2.3. Schematic diagram of the preconcentration set-up for capture of carbonyl compounds in e-cig. Aerosols. 


\subsection{RESULTS AND DISCUSSION}

To understand the contributions of thermal decomposition of PG or VG (physical properties in Table 2.1) to carbonyl generation and for comparison with carbonyl generation from commercial e-liquids, we measured carbonyl compounds in aerosols made from neat PG (100\%), neat VG (100\%), and mixtures, e.g., 50:50. The amounts of acetaldehyde, acetone, acrolein and formaldehyde were measured as a function of the percentage of PG in the mixtures (Figures 2.4 and 2.5). Fluid consumption varied from 6.0 to $11.5 \mathrm{mg} /$ puff at the puff volume of $91 \mathrm{~mL}$. Neat PG generated the highest level of acetaldehyde $(1.01 \pm 0.34 \mu \mathrm{g} / \mathrm{puff})$, then formaldehyde $(0.25 \pm 0.12 \mu \mathrm{g} / \mathrm{puff})$, acetone $(0.11 \pm 0.007 \mu \mathrm{g} /$ puff $)$ and low levels of crotonaldehyde yet above LoD $(0.25 \pm 0.12 \mathrm{ng} / \mathrm{puff})$. The acrolein level was below the limit of detection (LoD, estimated $<0.03 \mathrm{ng} /$ puff). Neat VG produced acetaldehyde $(0.70 \pm 0.03 \mu \mathrm{g} / \mathrm{puff})$, formaldehyde $(0.59 \pm 0.11 \mu \mathrm{g} / \mathrm{puff})$, acetone $(0.11 \pm 0.01 \mu \mathrm{g} / \mathrm{puff})$, acrolein $(0.08 \pm 0.002 \mu \mathrm{g} / \mathrm{puff})$ but not crotonaldehyde.

\subsubsection{Carbonyls in aerosols of commercial e-liquids with flavorants}

This work measured generation of carbonyl compounds from commercial eliquids with flavoring chemicals using "tank type" refillable e-cigarette devices to generate aerosols. Acetaldehyde and formaldehyde levels in aerosols of Classic Tobacco flavor e-liquid were lower than both neat PG and VG, while acrolein was below the LoD. Formaldehyde levels in aerosols of the other three Set I e-liquids (Magnificent Menthol, Vivid Vanilla, and Cherry Crush e-cigs (blu $\left.{ }^{\circledR}\right)$ ) were in the range of formaldehyde generated from neat PG to neat VG, while acetaldehyde levels in aerosols of the other 
three e-liquids were lower than the levels generated from neat PG and VG (Figure 2.6). Acrolein in aerosols from the other 3 e-liquids was higher than that in aerosols from pure VG (Figure 2.4). Aldehydes also were measured in aerosols from three Set II e-liquids (Menthol Ice, Mocha Café and Southern Classic). Menthol and Southern Classic e-liquids generated much higher levels of formaldehyde, acetaldehyde and acrolein in aerosols than did pure PG or VG or any PG:VG mixtures (Figure 2.7). Formaldehyde in aerosols from Menthol e-liquid was 8.1-times greater than that produced from pure PG and 3.4times greater than that of pure VG, while formaldehyde in aerosols from Southern Classic e-liquid was 8.7 times that of pure PG and 3.7 times that of pure VG. The 3-8 fold increases of acetaldehyde, formaldehyde, acrolein and levels in the derived aerosols from Set II (Menthol and Southern Classic) e-liquids in comparison with pure PG and VG likely were induced by decomposition of the flavoring chemicals. GC-MS analysis indicated that the level of propionaldehyde was much lower $(<1 / 20$; give value) than the levels of acetone, formaldehyde and acetaldehyde, thus propionaldehyde. Hydroxylacetone also was detected in aerosol of both neat PG and VG (Figure 2.4). As can be expected based on molecular structure (Scheme 2.3), the thermally induced degradation of PG generated a higher level of acetaldehyde whereas that of VG generated a higher level of formaldehyde. The levels of these carbonyl compounds in aerosols were at their lowest points when generated from the mixture at $25 \mathrm{wt} \%$ of PG compared with all other mixtures. These lowest levels may be induced by a combination of mass transfer and heat transfer related the mixture of $25 \mathrm{wt} . \%$ of PG. As the percentage of PG increased from 25 to $100 \mathrm{wt} . \%$, the levels of acetone, acetaldehyde and formaldehyde also increased, while acrolein levels decreased to below the LoD). The detection of hydroxyl-acetone provides 
experimental data to support the theoretical mechanisms (Scheme 2.3). No carbonyl compounds were detected in unvaped neat propylene glycol and glycerol and this proved that the carbonyl compounds detected in the aerosols were formed as a result of the thermal decomposition of the humectants.

Table 2.1. Physical properties of propylene glycol (PG) and vegetable glycerol (VG).

\begin{tabular}{|c|c|c|c|c|c|c|}
\hline & $\begin{array}{c}\text { Chemical } \\
\text { Formula }\end{array}$ & $\begin{array}{c}\mathrm{Mw} \\
(\mathrm{g} / \mathrm{mol})\end{array}$ & $\begin{array}{c}\text { Density } \\
\left(\mathrm{g} / \mathrm{cm}^{3}\right)\end{array}$ & $\begin{array}{c}\text { B.P. } \\
\left({ }^{\circ} \mathrm{C}\right)\end{array}$ & $\begin{array}{c}\text { Viscosity }(\mathrm{Pa} \cdot \mathrm{s}) \\
\text { at } 25{ }^{\circ} \mathrm{C}\end{array}$ & $\begin{array}{c}\text { Vapor Pressure } \\
\text { at } 200{ }^{\circ} \mathrm{C}\end{array}$ \\
\hline $\mathrm{PG}$ & $\mathrm{C}_{3} \mathrm{H}_{8} \mathrm{O}_{2}$ & 76.10 & 1.036 & 188.2 & 0.042 & $1030 \mathrm{mmHg}$ \\
\hline VG & $\mathrm{C}_{3} \mathrm{H}_{8} \mathrm{O}_{3}$ & 92.09 & 1.261 & 290 & 1.412 & $46 \mathrm{mmHg}$ \\
\hline
\end{tabular}

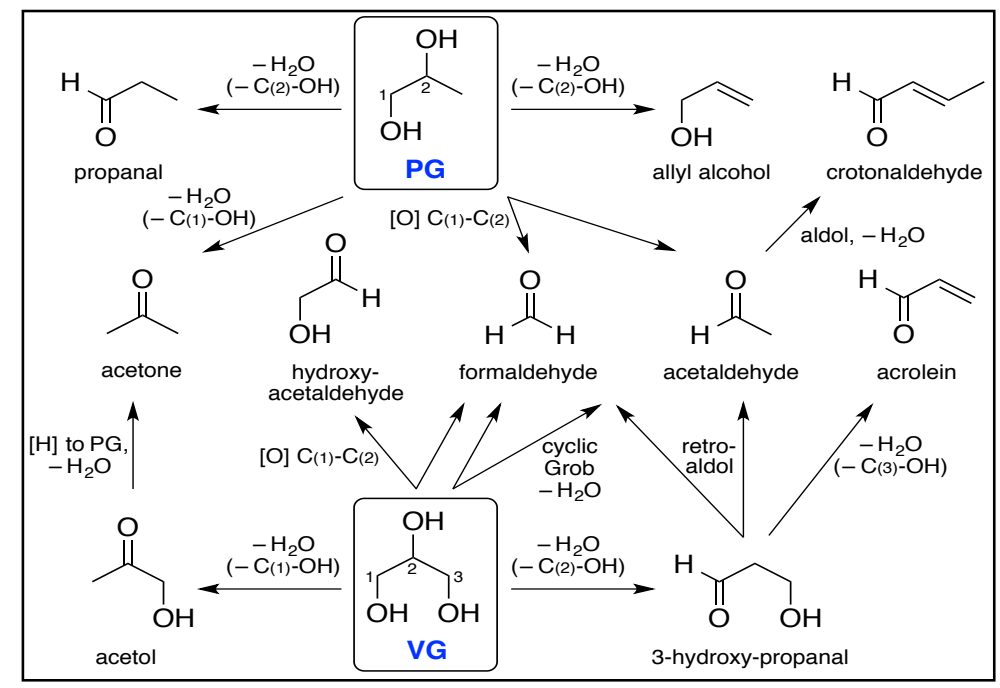

Scheme 2.3. Proposed pyrolysis of vegetable glycerol (VG) and propylene glycol (PG) humectants inelectronic cigarette aerosols. 


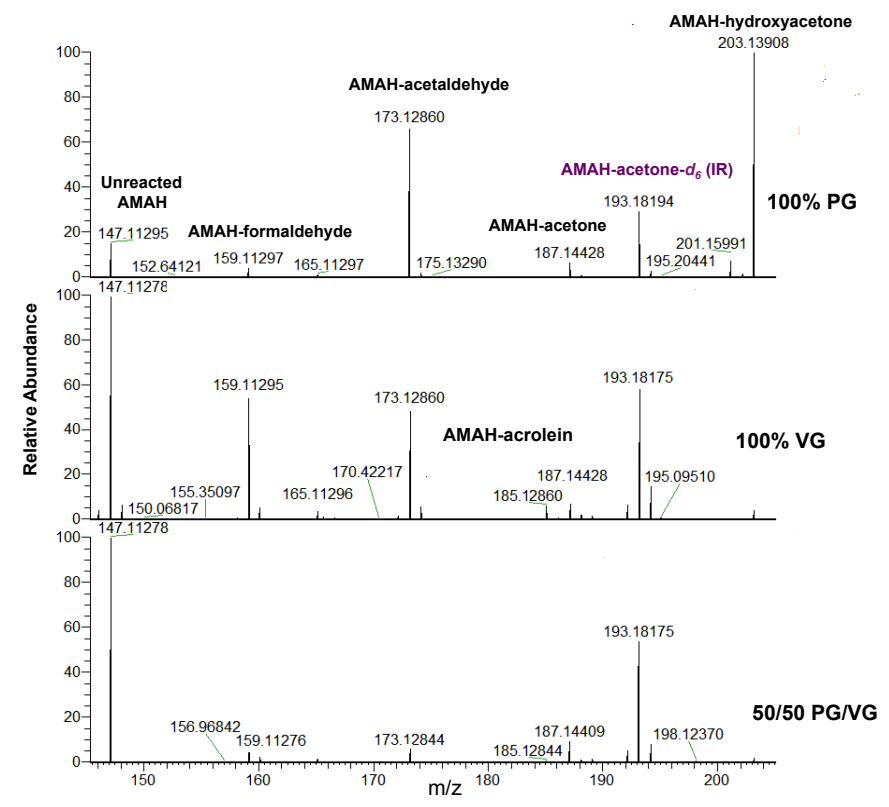

Figure 2.4. The FT-ICR-MS of the aerosols of $100 \%$ PG, $100 \%$ VG and PG/VG in 50/50 ratio showing different carbonyl compounds formed.
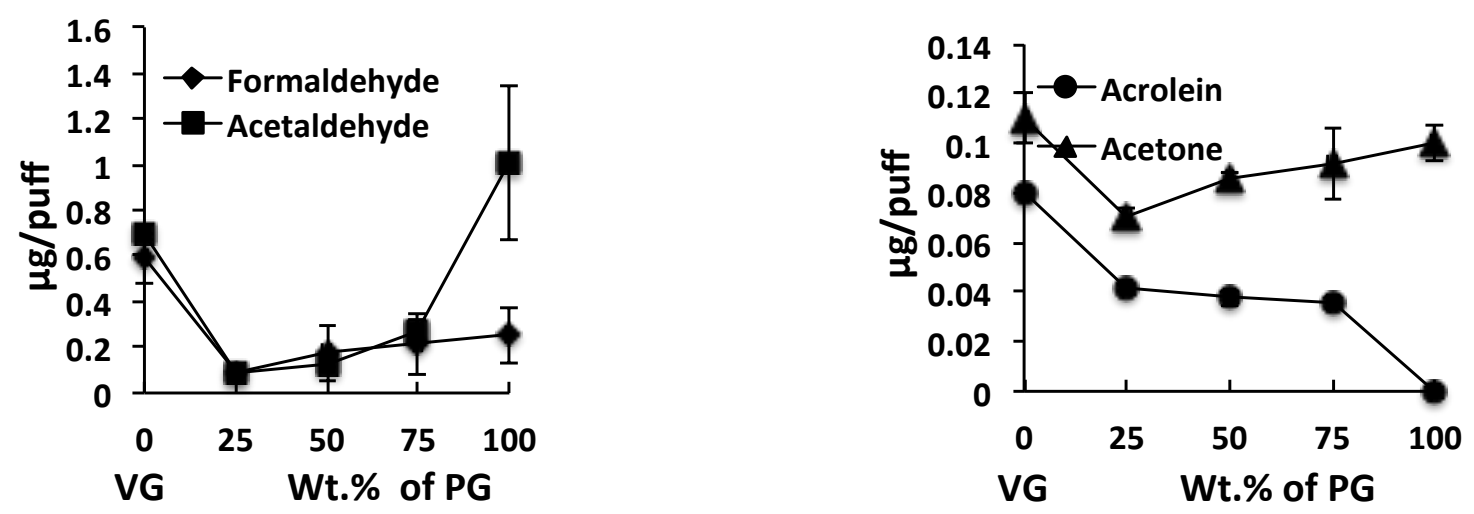

Figure 2.5. Amounts of formaldehyde, acetaldehyde, acrolein and acetone as a function of the percentage of propylene glycol PG) in the mixtures of vegetable glycerin (VG) and propylene glycol. Error bars represent one standard deviation of triplicte measurements $(\mathrm{N}=3)$. 


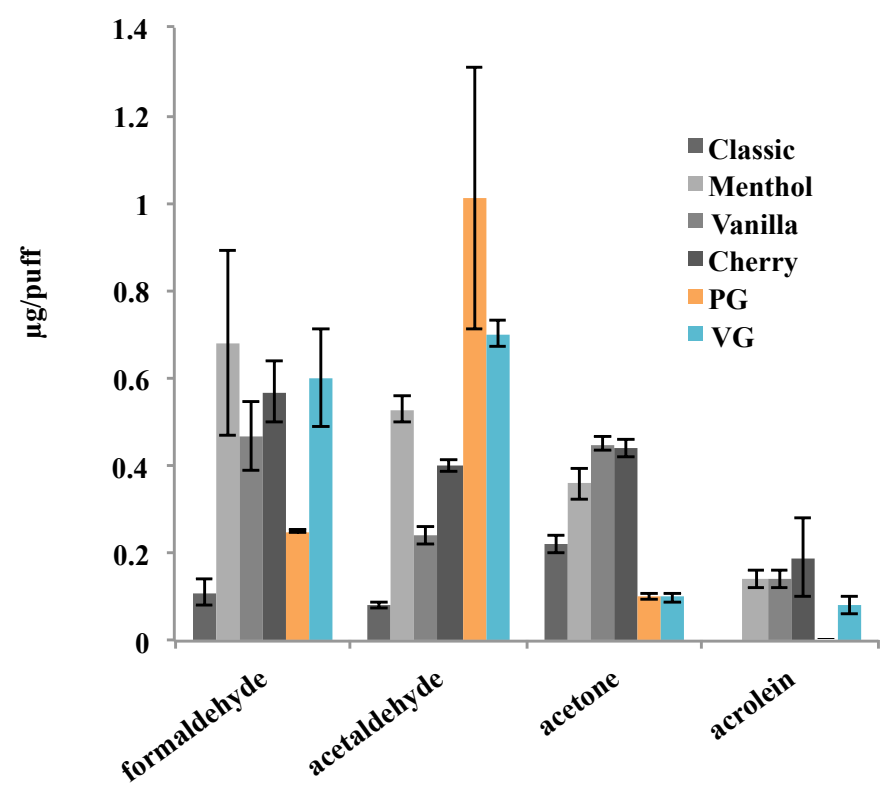

Figure 2.6. Amounts of formaldehyde, acetaldehyde, acrolein and acetone as a generated while vaping a set (I) of e-cigarrettes as compared with neat PG and neat VG. Error bars represent one standard deviation of triplicte measurements $(\mathrm{N}=3)$.

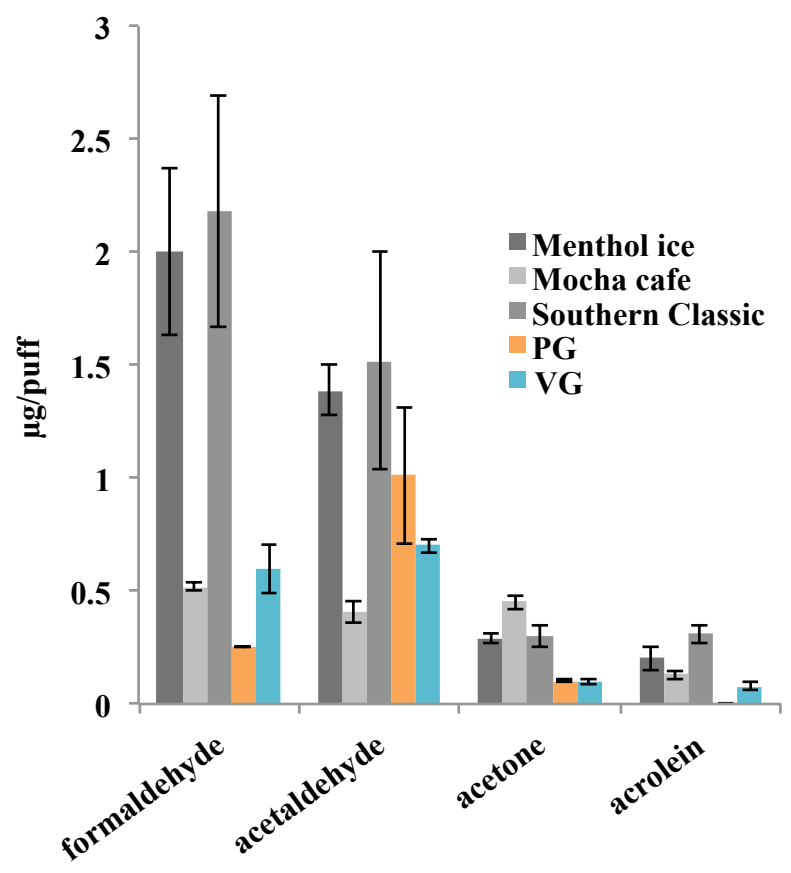

Figure 2.7. Amounts of formaldehyde, acetaldehyde, acrolein and acetone as a generated while vaping another set (II) of e-cigarrettes as compared with neat PG and neat VG. Error bars represent one standard deviation of triplicte measurements $(\mathrm{N}=3)$. 


\subsubsection{Generation of carbonyls from e-cigarettes aerosols: effects of battery power output}

Aerosols generated from 10 puffs (puff duration of $4 \mathrm{sec}$, puff volume of 91 $\mathrm{mL} /$ puff, puff frequency of 2 puffs $/$ min) were collected in Tedlar bags using a softwarecontrolled (FlexiWare) cigarette-smoking robot (CSR) (Sci-Req, Montreal, CAN) as described earlier. The puff duration, puff volume and puff frequency in this study are within the ranges used by e-cigarette users. ${ }^{114-115}$ To study the effect of puffing topography on emission of aldehydes in aerosols, polypropylene syringes with a $60 \mathrm{~mL}$ capacity were also used to collect aerosols of e-cigarettes by manually varying puff duration and puff volume. While the first generation e-cigarette has a fixed battery voltage of $3.7 \mathrm{~V}$ (power: $4.6 \mathrm{~W}$ ), the battery power of the newer generation e-cigarette was tested at $9.1 \mathrm{~W}(3.7 \mathrm{~V}), 11.7 \mathrm{~W}(4.2 \mathrm{~V}), 14.7 \mathrm{~W}(4.7 \mathrm{~V})$ and $16.6 \mathrm{~W}(5.0 \mathrm{~V})$ for vaporization of e-liquids. For aerosols collected from e-liquids EL04-EL-06 (Table 2.3) at the battery power output of $14.7 \mathrm{~W}$ and $16.6 \mathrm{~W}$, the aerosol samples was diluted 50 times with $\mathrm{N}_{2}$ and then drawn through the microreactors because of much higher levels of generated aldehydes.

Because increasing battery power output of newer e-cigarette devices increases the atomizer coil temperature that results in emissions of more aldehydes in aerosols of eliquids, ${ }^{972636}$ we investigated the effect of the battery power output of the newer ecigarette devices on generation of carbonyl compounds in aerosols of e-liquids. EL04EL04 and EL05 generated much more carbonyl compounds at a power output of $11.7 \mathrm{~W}$ and above. Figure 2.8 shows GC-MS chromatograms of AMAH and its adducts from the aerosol samples collected from Halo Menthol Ice at vaporization power of $11.7 \mathrm{~W}$ (4.2 V) $14.7 \mathrm{~W}(4.7 \mathrm{~V})$ and $16.6 \mathrm{~W}(5.0 \mathrm{~V})$. Acetaldehyde, acrolein, formaldehyde, 
propionaldehyde and butyraldehyde were detected. Increasing the power from $11.7 \mathrm{~W}$ to 16.6 W resulted in dramatic increases of the levels of these aldehydes (Table 2.2).

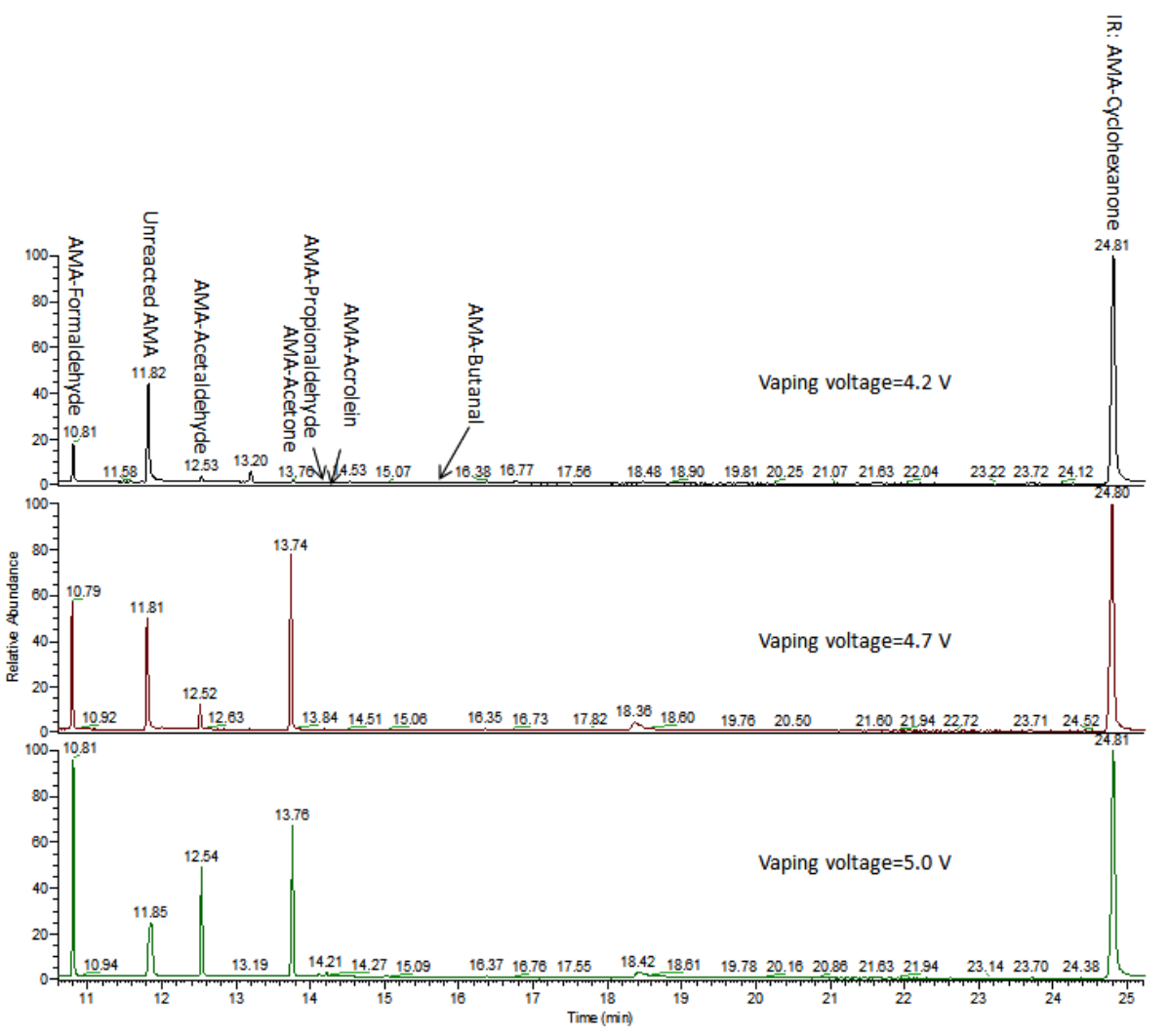

Figure 2.8. Representative GC-MS chromatograms of aldehydes. The newer e-cigarette device (iTaste) was used to vaporize e-liquid Halo Mentol Ice at a battery power output of $11.7 \mathrm{~W}(4.2 \mathrm{~V}), 14.7 \mathrm{~W}(4.7 \mathrm{~V})$, and 16.6W (5.0V).

Similar results of dramatic increases of acetaldehyde, acrolein and formaldehyde with increasing vaping power output to $9 \mathrm{~W}$ and above have been reported. ${ }^{116}$ Higher power results in overheating of the coil and leads to excessive aldehyde generation by thermal decomposition of humectants (“dry puff" condition). ${ }^{116}$ 
Table 2.2. Effect of varying battery power output on generation of aldehydes in the aerosols from e-liquid Halo Menthol Ice.*

\begin{tabular}{|c|c|c|c|c|c|c|}
\hline $\begin{array}{l}\text { Voltage } \\
\text { (W) }\end{array}$ & $\begin{array}{l}\text { Acetaldehyde } \\
\mu \mathrm{g} \\
(\mathrm{ppm})\end{array}$ & $\begin{array}{l}\text { Acrolein } \\
\mu \mathrm{g} \\
(\mathrm{ppm})\end{array}$ & $\begin{array}{l}\text { Formaldehyde, } \\
\mu \mathrm{g} \\
(\mathrm{ppm})\end{array}$ & $\begin{array}{l}\text { Acetone } \\
\mu \mathrm{g} \\
(\mathrm{ppm})\end{array}$ & $\begin{array}{l}\text { Propionalde } \\
\text { hyde } \mu \mathrm{g}, \\
(\mathrm{ppm})\end{array}$ & $\begin{array}{l}\text { Butyrald } \\
\text { ehyde, } \mu \mathrm{g} \\
(\mathrm{ppm})\end{array}$ \\
\hline $11.7 \mathrm{~W}$ & $\begin{array}{l}22.7 \pm 3.4 \\
(35.9 \pm 5)\end{array}$ & $\begin{array}{l}1.2 \pm 0.8 \\
(1.5 \pm 1.0)\end{array}$ & $\begin{array}{l}129.6 \pm 9.7 \\
(300.8 \pm 22.4)\end{array}$ & $\begin{array}{l}11.46 \pm 0.50 \\
(13.75 \pm 0.60)\end{array}$ & $\begin{array}{l}0.6 \pm 0.4 \\
(0.7 \pm 0.5)\end{array}$ & $\begin{array}{l}0.5 \pm 0.3 \\
(0.5 \pm 0.3)\end{array}$ \\
\hline $14.7 \mathrm{~W}$ & $\begin{array}{l}134.30 \pm 7.8 \\
(212.33 \pm 12.3)\end{array}$ & $\begin{array}{l}3.2 \pm 0.7 \\
(3.9 \pm 0.9)\end{array}$ & $\begin{array}{l}386.8 \pm 11.0 \\
(898.1 \pm 26)\end{array}$ & $\begin{array}{l}984.92 \pm 50.10 \\
(1181.54 \pm 60.10)\end{array}$ & $\begin{array}{l}3.4 \pm 1.5 \\
(4.0 \pm 1.8)\end{array}$ & $\begin{array}{l}5.0 \pm 8.5 \\
(4.8 \pm 8.2)\end{array}$ \\
\hline $16.6 \mathrm{~W}$ & $\begin{array}{l}532.1 \pm 60.2 \\
(841.3 \pm 95.2)\end{array}$ & $\begin{array}{l}16.2 \pm 0.3 \\
(20.3 \pm 0.4)\end{array}$ & $\begin{array}{l}819.81 \pm 76.8 \\
(1902.8 \pm 178.3)\end{array}$ & $\begin{array}{l}808.72 \pm 72.6 \\
(970.17 \pm 87.1)\end{array}$ & $\begin{array}{l}17.9 \pm 0.9 \\
(21.4 \pm 10)\end{array}$ & $\begin{array}{l}13.6 \pm 0.5 \\
(13.1 \pm 0.5)\end{array}$ \\
\hline
\end{tabular}

* A total of 10 puffs of aerosol was collected at the puff volume of $91 \mathrm{~mL}$, puff duration of $4 \mathrm{~s}$. Each experiment was performed in triplicate and the data are expressed as the average $[ \pm \mathrm{SD}]$ of the measured values.

To quantify the fraction of aldehydes that reacted with propylene glycol and/or glycerin to form hemiacetals in the aerosols of e-cigarettes, we collected aerosolized eliquids in NMR tubes containing DMSO- $d_{6}$ using the newer e-cigarette device. We initially attempted to detect the formation of a formaldehyde-derived hemiacetal in aerosols of all e-cigarettes in table 2.3. No formaldehyde hemiacetal signal was detected in any of the aerosols generated from the first generation blu e-cigarettes with all tested puff volume and puff duration scenarios (data not shown). No hemiacetal was detectable in the aerosols of EL01, EL02 and EL03 at all battery power output from $9.1 \mathrm{~W}$ to 16.6 $\mathrm{W}$ as shown in figure 2.9 (a). In a positive control experiment, formaldehyde gas was introduced into EL01 e-liquid and under this condition a triplet signal at $\delta 6.18 \mathrm{ppm}$ and a doublet signal at $\delta 4.61 \mathrm{ppm}$ were observed, confirming formation of a formaldehyde hemiacetal (Fig. 2.9(b)). Thus, the lack of detectable formaldehyde hemiacetal in aerosols of the first generation e-cigarette and e-liquids EL01 to EL03 was likely related to the 
generally low amount of formaldehyde present in these aerosols, even when at higher battery power output.

Table 2.3. Characteristics of e-cigarette cartridges and e-liquids used in this study.

\begin{tabular}{|c|c|c|c|c|}
\hline $\begin{array}{l}\text { Product } \\
\text { code }\end{array}$ & Brand name & Type & $\begin{array}{l}\text { Nicotine } \\
\text { content } \\
\text { (label) }\end{array}$ & Manufacturer \\
\hline EC01 & $\begin{array}{ll}\text { blu } & \text { Classic } \\
\text { Tobacco } & \end{array}$ & Cartridge & $16 \mathrm{mg}$ & $\begin{array}{l}\text { Imperial } \\
\text { Tobacco }\end{array}$ \\
\hline EC02 & $\begin{array}{l}\text { blu Magnificent } \\
\text { Menthol }\end{array}$ & Cartridge & $16 \mathrm{mg}$ & $\begin{array}{l}\text { Imperial } \\
\text { Tobacco } \\
\end{array}$ \\
\hline EC03 & $\begin{array}{l}\text { blu } \\
\text { Vanilla }\end{array}$ & Cartridge & $16 \mathrm{mg}$ & $\begin{array}{l}\text { Imperial } \\
\text { Tobacco }\end{array}$ \\
\hline EC04 & Cherry & Cartridge & $16 \mathrm{mg}$ & $\begin{array}{l}\text { Imperial } \\
\text { Tobacco }\end{array}$ \\
\hline EL01 & $\begin{array}{l}\text { eVo Black } \\
\text { diamond }\end{array}$ & e-liquid & $6 \mathrm{mg} / \mathrm{mL}$ & $\begin{array}{l}\text { Nicopure } \\
\text { Lab USA }\end{array}$ \\
\hline EL02 & Smooththol & e-liquid & $6 \mathrm{mg} / \mathrm{mL}$ & NicQuid \\
\hline EL03 & $\begin{array}{l}\text { Perfected } \\
\text { Vape/Clearwater }\end{array}$ & e-liquid & $6 \mathrm{mg} / \mathrm{mL}$ & $\begin{array}{l}\text { Delaware } \\
\text { Vapor USA }\end{array}$ \\
\hline EL04 & $\begin{array}{ll}\text { Halo } & \text { Café } \\
\text { Mocha } & \\
\end{array}$ & e-liquid & $6 \mathrm{mg} / \mathrm{mL}$ & Halo USA \\
\hline EL05 & $\begin{array}{l}\text { Halo Menthol } \\
\text { Ice }\end{array}$ & e-liquid & $6 \mathrm{mg} / \mathrm{mL}$ & Halo USA \\
\hline EL06 & $\begin{array}{l}\text { Halo Southern } \\
\text { Classic }\end{array}$ & e-liquid & $6 \mathrm{mg} / \mathrm{mL}$ & Halo USA \\
\hline
\end{tabular}
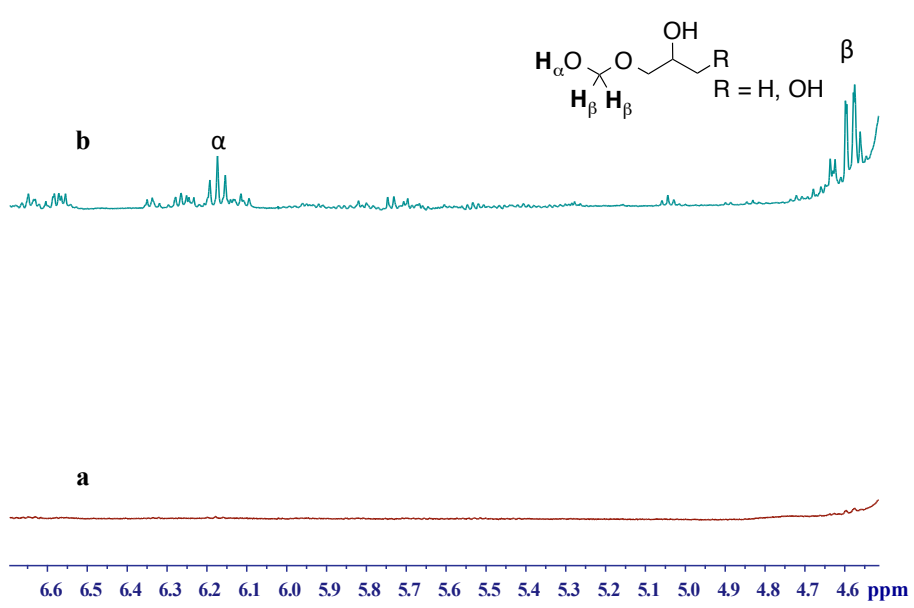

Figure 2.9. ${ }^{1} \mathrm{H}$ NMR spectra (DMSO- $d_{6}$ ) for detection of hemiacetals, (a) e-liquid EL01 vaporization at the battery power output of $16.6 \mathrm{~W}$ (no hemiacetal detected); (b) unvaporized EL01 spiked with formaldehyde. 


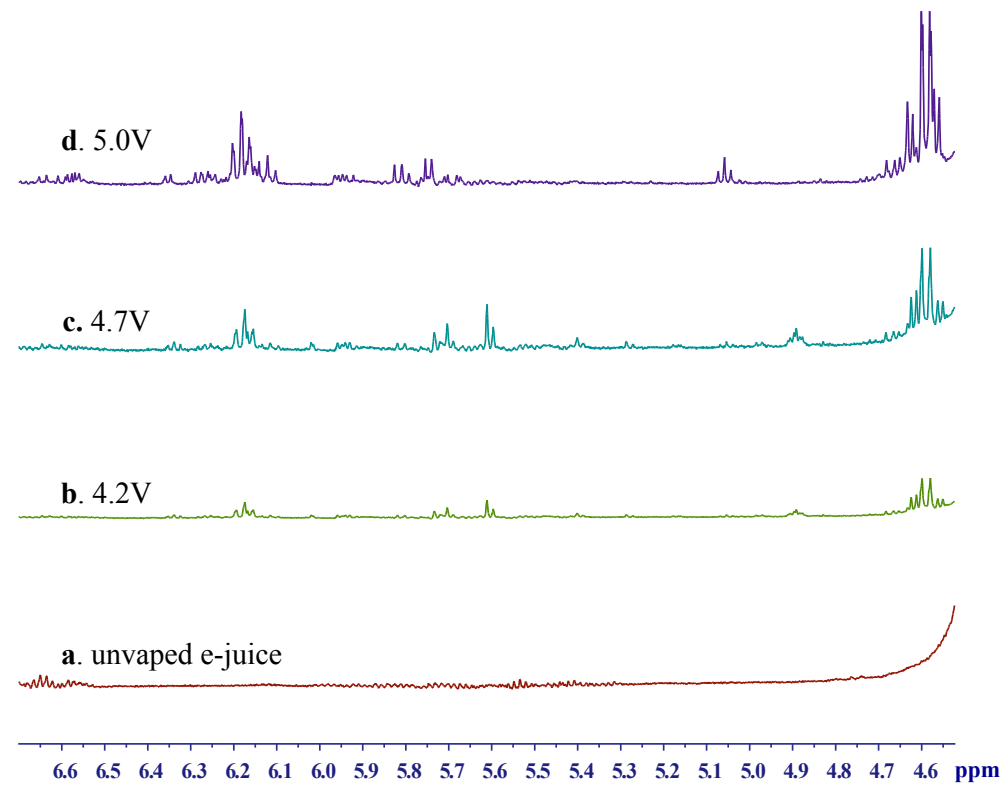

Figure 2.10. ${ }^{1} \mathrm{H}$ NMR spectra (DMSO- $d_{6}$ ) of hemiacetals, (a) unvaporized e-liquid EL05; the tank type e-cigarette (iTaste) was used to vaporize e-liquid EL05 at a battery power output of (b) $11.7 \mathrm{~W}(4.2 \mathrm{~V}),(\mathrm{c}) 14.7 \mathrm{~W}(4.7 \mathrm{~V})$, and (d) $16.6 \mathrm{~W}(5.0 \mathrm{~V})$.

Formaldehyde hemiacetal in aerosols of EL05 was detected at the battery power output from $11.7 \mathrm{~W}$ to $16.6 \mathrm{~W}$ by NMR. No other aldehyde hemiacetal such as acetaldehyde hemiacetal and acrolein hemiacetal was detected. Figure 2.10 shows that there was an increase in peak intensity of the hemiacetal as the e-cigarette battery power output was increased from $11.7 \mathrm{~W}(4.2 \mathrm{~V})$ to $16.6 \mathrm{~W}(5.0 \mathrm{~V})$. At a battery power output below 11.7 W, formaldehyde hemiacetal was below the limit of detection. The amounts of formaldehyde hemiacetal in aerosol increased as the power was increased and the calculated amounts (mean $\pm \mathrm{SD}$ ) of hemiacetal were based on the internal standard (Figure 2.11). 


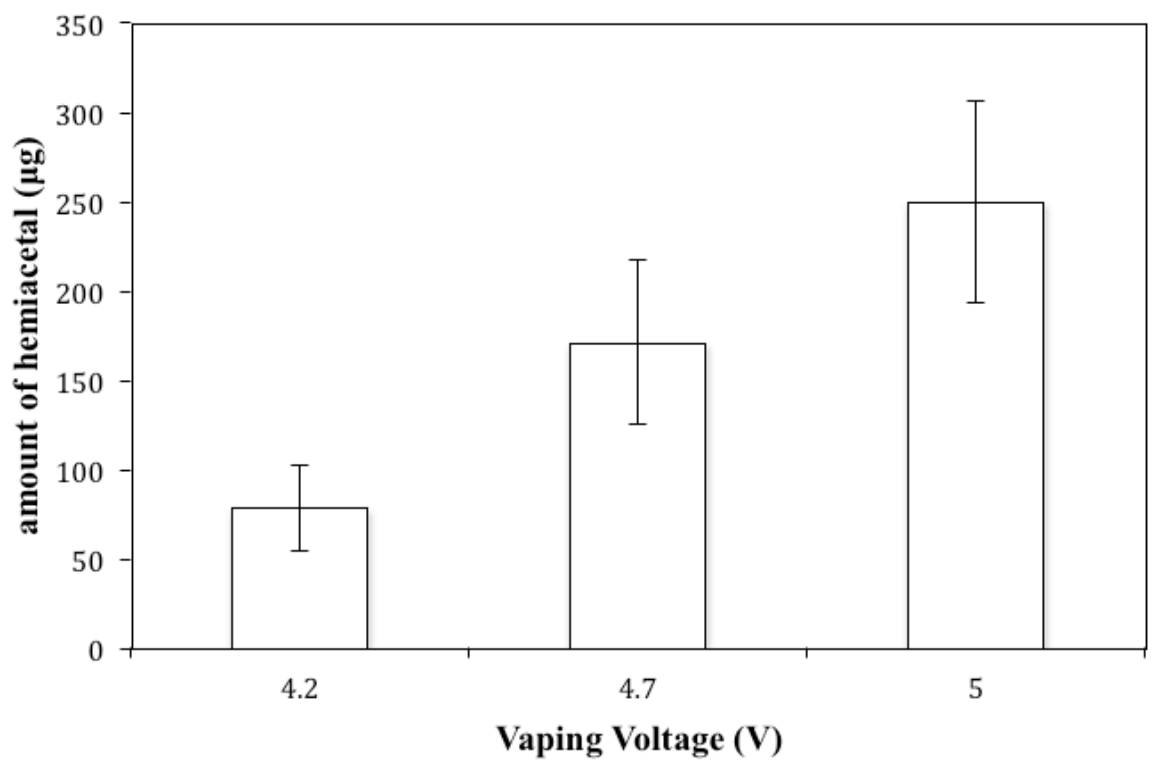

Figure 2.11. The relationship between the amounts of formaldehyde-hemiacetal in 10 puffs of aerosols collected from e-liquid EL05 and the battery power output of $11.7 \mathrm{~W}$, $14.7 \mathrm{~W}$ and $16.6 \mathrm{~W}$.

At a battery power output of $11.7 \mathrm{~W}, 78.6 \pm 23.8 \mu \mathrm{g} / 10$ puffs of formaldehyde hemiacetal was measured, whereas at $16.6 \mathrm{~W}, 250.4 \pm 56.1 \mu \mathrm{g} / 10 \mathrm{puffs}$ of the hemiacetal was measured. We were able to estimate the amount of the bound formaldehyde as $22.2 \pm 6.7$ $\mu \mathrm{g}$ and $70.7 \pm 15.8 \mu \mathrm{g}$ from the measured formaldehyde hemiacetal at $11.7 \mathrm{~W}$ and $16.6 \mathrm{~W}$, respectively. These amounts of formaldehyde could be released from reversible reaction of formaldehyde hemiacetal. Much higher formaldehyde of 380 (puff volume of $50 \mathrm{~mL}$ for 10 puffs) was reported from formaldehyde-hemiacetal at the e-cigarette voltage output of $5 \mathrm{~V}$ for vaporization of the e-liquid EL04. ${ }^{71}$ Given the puff volume of $35 \mathrm{~mL}$, we could estimate that the formaldehyde in formaldehyde-hemiacetal could be approximately $44.6 \%$ of free formaldehyde at the power output of $11.7 \mathrm{~W}$ and approximately $22.4 \%$ of free formaldehyde at the power output of $16.6 \mathrm{~W}$. Higher formaldehyde levels of 380 (puff volume of $50 \mathrm{~mL}$ for 10 puff s) was reported from 
formaldehyde-hemiacetal at the e-cigarette voltage output of $5 \mathrm{~V}$ for vaporization of EL04 e-liquid.

\subsection{CONCLUSION}

Our experimental results have shown that aldehydes are formed from heating of humectants and flavorants in e-cigarette liquids. Mechanisms to form these carbonyl

compounds have been proposed previously. ${ }^{117-118}$ The detection of hydroxyl-carbonyl intermediates (e.g., acetol, hydroxyl-acetaldehyde, 3-hydroxy-propanal) in this study provides experimental data in support of the theoretical mechanisms. ${ }^{118-119}$ Regardless of the paths to aldehyde generation, our research indicates that the percentages of PG and VG as well as added flavorants to e-liquids affect formation of these aldehydes in aerosols. As can be expected based on molecular structure, VG generated higher levels of formaldehyde and acrolein, whereas PG generated a higher level of acetaldehyde. Lower levels of carbonyls were generated from mixtures of the humectants, notably $25 \%$ PG: $75 \% \mathrm{VG}$, likely due to multiple factors including mass transfer and heat transfer as well as rate-determining dehydration kinetics associated with the mixture composition. In order to understand the specific contribution of flavorants to generation of carbonyl compounds and formaldehyde-hemiacetal, further studies involving aerosolization of humectants laced with each flavorant and subsequent analysis of the aerosols for carbonyl compounds will be necessary. The chemistry of additives with e-liquids when subjected to a high temperature needs investigation. Also, formaldehyde-hemiacetals was generated during aerosolization of some e-liquid brands. The potential toxicity of the hemiacetal is not yet understood. The ability of the hemiacetal to convert to formaldehyde after 
inhalation by humans therefore needs to be investigated. Heavy metals like cadmium, chromium, lead, manganese, and, nickel have been reported in electronic liquid and derived aerosols. ${ }^{120-121}$ Some of these are transition metals are used as catalysts in organic reactions. Therefore, effects of different metals on generation on generation of carbonyl compounds in electronic cigarette aerosolization also need to be investigated.

\subsection{EXPERIMENTAL}

A customized e-cigarette holder was regulated by a software-controlled (FlexiWare) system (Sci-Req, Montreal, CAN) for generation of aerosols. The puffing protocol consisted of a $4 \mathrm{~s}$ puff duration, a $91 \mathrm{~mL}$ puff volume, and a $56 \mathrm{~s}$ puff interval to mirror typical e-cigarette user puffing topography. ${ }^{31-32}$ Neat PG $(100 \%)$, neat VG and PG and VG mixtures (PG: VG, 25:75; 50:50, 75:25 wt\%) were used for aerosol generation. For comparison, commercial e-liquids (described above) were used in e-cigarette aerosol generation. The e-cigarette battery (bluPLUS+) power output was 7.6 watts $(3.7 \mathrm{~V})$ in all experiments. Batteries were fully charged overnight before use.

Details of the microreactor have been published elsewhere. ${ }^{47,54}$ Micropillar surfaces in the microreactor were functionalized by infusion of a solution of AMAH $\left(1 \times 10^{-6} \mathrm{~mol}\right.$; 4-(2-aminooxyethyl)-morpholin-4-ium chloride $\left(\mathrm{AMAH}^{55}\right)$ in methanol followed by evaporation of the solvent in a vacuum oven at $40{ }^{\circ} \mathrm{C}$. Fused silica capillary tubes (350 $\mu \mathrm{m}$ o.d., $250 \mu \mathrm{m}$ i.d.) were connected to the inlet and outlet ports of the microreactor, respectively, with a silica-based bonding agent.

To capture carbonyl compounds for analysis, aerosol samples were collected in Tedlar bags and evacuated through the microreactors by a vacuum $(3.5 \mathrm{~mL} / \mathrm{min})$. After 
the evacuation process, the microreactors were eluted with $150 \mu \mathrm{L} \mathrm{MeOH}$ followed by addition of an internal reference (IR). For FT-ICR-MS analyses, the AMAH-deuterated acetone adduct was added as the IR. For GC-MS analysis, AMAH-cyclohexanone $\left(1 \times 10^{-7} \mathrm{~mol}\right)$ was chosen because it is symmetrical (i.e., no geometrical isomers for the AMAH-cyclohexanone adduct) and because cyclohexanone was undetected in e-cigarette aerosols. GC-MS was used to quantitate isomeric compounds reacted with AMAH, e.g., acetone and propionaldehyde. Calibration curves of internal standards for all detected carbonyls were established for quantitative measurements in both FT-ICR-MS and GCMS. For GS-MS analyses, poly-4-vinylpyridine (PVP, $5 \mathrm{mg}$ ) was added to the eluted solutions to convert positively charged AMAH adducts to neutral AMA adducts (Scheme 2.4). ${ }^{55}$ The suspension was vortex-mixed for $30 \mathrm{~s}$ and allowed to stand for $30 \mathrm{~min}$ for the sedimentation of PVP particles, after which a $20 \mu \mathrm{L}$ aliquot solution was used for GCMS analysis.

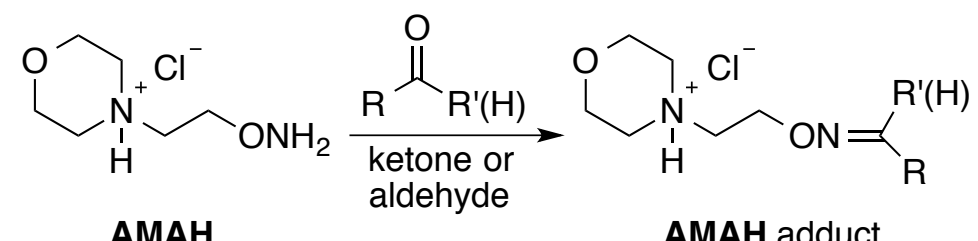

AMAH

AMAH adduct

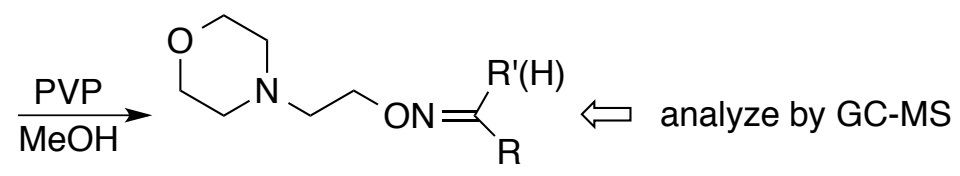

AMA adduct

Scheme 2.4. Microreactor oximation of carbonyl compounds by AMAH and neutralization of adducts with PVP prior to GC-MS analyses.

\subsubsection{FT-ICR-MS}

An FT-ICR-MS instrument (Finnigan LTQ-FT, Thermo Electron, Bremen, Germany) equipped with a TriVersa NanoMate ion source (Advion BioSciences, Ithaca, 
NY) fitted with an electrospray chip (nozzle inner diameter $5.5 \mu \mathrm{m}$ ) was used for all mass spectral analyses. The TriVersa NanoMate was operated in positive ion mode by applying $2.0 \mathrm{kV}$ with no head pressure. Initially, low-resolution MS scans were acquired for $1 \mathrm{~min}$ to ensure the stability of ionization, after which high mass accuracy data were collected using the FT-ICR analyzer, where MS scans were acquired for 5 min and at the target mass resolution of 100,000 at $200 \mathrm{~m} / \mathrm{z}$.

Using AMAH-deuterated acetone adduct as internal reference (IR), the linearities of standard AMAH-carbonyls were first established by calibration curves built by plotting the ratio of intensity of AMAH-carbonly to intensity IR against of peak area of analytes to IS ( $\mathrm{I}_{\text {carbonyl }} / \mathrm{I}_{\mathrm{IR}}$ ) versus the ratio of the amount (mole) of the AMAH-carbonly to amount of IR ( $\left.\mathrm{M}_{\text {carbonyl }} / \mathrm{M}_{\mathrm{IR}}\right)$ (Figure 2.12). Linear regression was used to determine the slope, intercept, and coefficient of determination $\left(\mathrm{R}^{2}\right)$. The calibrations curved were used to quantify the carbonyl compounds of interest generated during aerosolization.

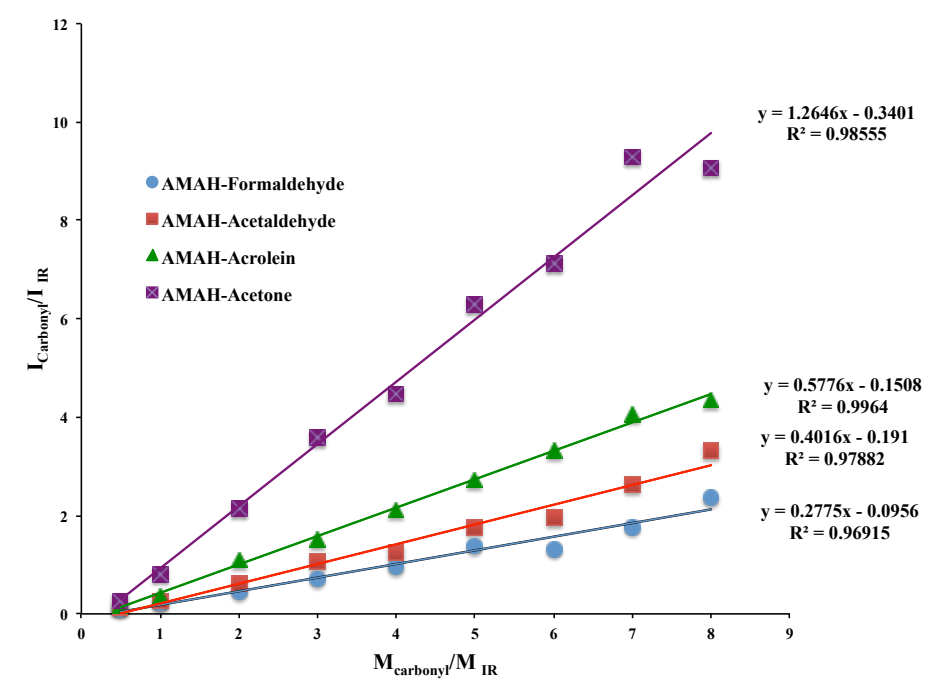

Figure 2.12. The calibration curves of standard AMAH-carbonyl compounds. The ration of Intensity of carbonlys to Intensity of Internal Reference (IR) was plotted against the ratio of amount of carbonlys to amount of IR. 


\subsubsection{NMR analysis of hemiacetals}

Ten puffs (puff volume: $35 \mathrm{~mL}$; puff duration: $4 \mathrm{~s}$, puff frequency of 1 puff/min) of the aerosols generated by the first generation e-cigarette and the "tank type" e-cigarette were collected using a $60 \mathrm{~mL}$ capacity polypropylene syringe with a very short rubber tube to connect the e-cigarette. After collection, the rubber tube and e-cigarette were immediately removed, and then the syringe was fitted with a long stainless needle to transfer the aerosolized liquid into an NMR test tube in an ice bath. During the transfer, most of the aerosol condensed and was collected as liquid. $400 \mu \mathrm{L}$ of deuterated DMSO was added to the NMR tube followed by the addition of a known amount of benzene $\left(1.72 \times 10^{-6} \mathrm{~mol}\right)$ as an internal standard. Then, ${ }^{1} \mathrm{H}-\mathrm{NMR}$ spectra (referenced to TMS) were immediately taken at $400 \mathrm{MHz}$. To verify formation of formaldehyde-hemiacetal in e-liquid, formaldehyde was generated by heating 1,3,5-trioxane and $8 \mathrm{~N}$ sulfuric acid at $95{ }^{\circ} \mathrm{C}^{122}$ and then introduced as a gas into e-liquids. Formaldehyde-hemiacetal was quantified by relative integration against the known amount of benzene added as an internal standard.

\subsubsection{GC-MS analysis of carbonyl adducts}

A Thermo Scientific GC-MS instrument equipped with an AI 1310 automatic sampler, a TRACE 1310 GC with a split/splitless injector and an ITQ 1100 series ion trap MS was used for analysis. The GC had an Agilent J\&W DB-17ms column $(60 \mathrm{~m} \times 0.25$ $\mathrm{mm} \times 0.25 \mu \mathrm{m}$ film thickness). Carrier gas helium flow rate was $1.5 \mathrm{~mL} / \mathrm{min}$. Column temperature was $50{ }^{\circ} \mathrm{C}$ for $1 \mathrm{~min}$, then increased by $10^{\circ} \mathrm{C} / \mathrm{min}$ up to $160{ }^{\circ} \mathrm{C}$, and then to $200{ }^{\circ} \mathrm{C}$ by $2{ }^{\circ} \mathrm{C} / \mathrm{min}$. After that, the temperature was increased by $12{ }^{\circ} \mathrm{C} / \mathrm{min}$ up to $280{ }^{\circ} \mathrm{C}$ 
and was held at $280^{\circ} \mathrm{C}$ for $5 \mathrm{~min}$. The total running time was $41 \mathrm{~min}$.

The samples were split injected with split flow of $15 \mathrm{~mL} / \mathrm{min}$ and a slit ratio of 10 . 
CHAPTER 3

KINETIC STUDIES OF OXIMATION REACTION AND SYNTHESIS OF A CATIONIC HYDRAZINE REAGENT

3.1. INTRODUCTION OF OXIMATION REACTIONS

3.2. KINETIC STUDIES OF REACTION BETWEEN AMINOOXIES AND CARBONYLS

3.3. RESULTS AND DISCUSSION

3.4. HYDRAZINE SYNTHESIS

3.4. CONCLUSION

3.5. EXPERIMENTAL SECTION 


\subsection{INTRODUCTION OF OXIMATION REACTIONS}

The reaction between an aminooxy moiety $\left(\mathrm{RONH}_{2}\right)$ and the carbonyl group of an aldehyde or ketone- known as an oximation reaction- is a versatile click chemistry ${ }^{123-124}$ coupling that generates a robust oxime ether linkage. Carbonyl-selective derivatizing aminooxy agents have been used for detection of oxidized cellular metabolites. ${ }^{125-126}$ Moreover, oxime based chemistries have been used for efficient bioconjugation of proteins and polysaccharides for the preparation of conjugate vaccines, ${ }^{127}$ to generate homogeneously glycosylated proteins, to recombinantly produce protein-bearing tailored glycans at specific sites. ${ }^{128}$ The reaction is also used to selectively capture aldehyde and ketone metabolites directly from air, such as exhaled breath which has been used for noninvasive detection of lung cancer, ${ }^{31,47,129}$ Furthermore, it is used to ligate linker molecules to fluorophores and gold nanoparticles, ${ }^{130}$ to analyze ketones in crude oil and coal $\operatorname{tar}^{131}$ and to selectively ligate carbonyls for labeling glycoconjugates for microscopy. ${ }^{132}$

In addition, biocompatible click reactions have shown promise for in situ ligations and applications in living organisms. Carbonyl condensation reactions such as imine-, hydrazone-, and oxime-bond formation (Scheme 3.0), Staudinger and Diels-Alder reactions as well as azide-alkyne cycloadditions are used for situ ligation. The oxime ligation is of special interest because of its efficiency and chemoselectivity in aqueous systems under mild acidic conditions. It is compatible with most biomolecule functionalities and water is the only side-product formed in this process. ${ }^{133}$ 


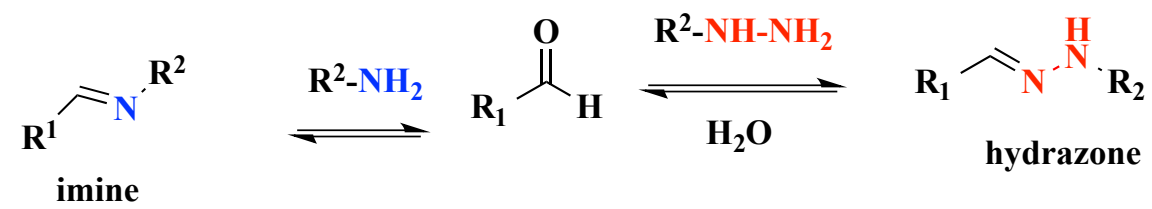

$$
\begin{aligned}
& \mathbf{H}_{2} \mathbf{O} \| \mathbf{R}^{2}-\mathrm{O}-\mathrm{NH}_{2} \\
& \mathbf{R}^{\mathbf{1}} \overbrace{\text { oxime }}^{\mathrm{N}} \cdot \mathbf{R}^{2}
\end{aligned}
$$

Scheme 3.0. Imine-, hydrazone-, and oxime-bond formation. $\mathrm{E}$ and $\mathrm{Z}$ isomers may be present depending on the nature of substituents and conditions.

Atmospheric carbonyl compounds are either derived from direct emissions or produced as reaction intermediates from oxidation of hydrocarbons initiated by $\mathrm{OH}$ radicals and ozone. ${ }^{134-135}$ Carbonyl compounds also play a central role in atmospheric chemistry close to the tropopause, and this is directly relevant to issues such as the assessment of the impact of air traffic and ozone depletion. ${ }^{136}$ It is thus crucial to understand the reactions and kinetics of carbonyl compounds particularly with respect to derivative formation for purposes of monitoring or analyses.

\subsection{KINETIC STUDIES OF REACTION BETWEEN AMINOOXIES AND CARBONYLS}

In spite of the importance of oximation reactions in the analysis of trace carbonyl compounds in air, exhaled breath and bio-liquids, very little has been done to study the reaction kinetics of aminooxy compounds with aldehydes and ketones. The oximation reaction kinetics of aminooxy compounds is very important for quantitative analysis of trace carbonyl compounds. In this thesis, we determined the reaction kinetics between quaternary ammonium aminooxy salts 2 -(aminooxy)- $N, \quad N, \quad N$-trimethylethan-1- 
ammonium iodide (ATM), 2-(aminooxy)- $N, \quad N$-dimethylethan-1-aminium chloride $(\mathrm{ADMH})$, and 4-(2-(aminooxy)ethyl)-morpholin-4-ium chloride (AMAH) (Scheme 3.1) and some selected carbonyl aldehydes and ketones. We used FT-ICR-MS to study the kinetics of oximation because of its ability measure fast reaction rates that are otherwise difficult or impossible to monitor using other spectrometric techniques.
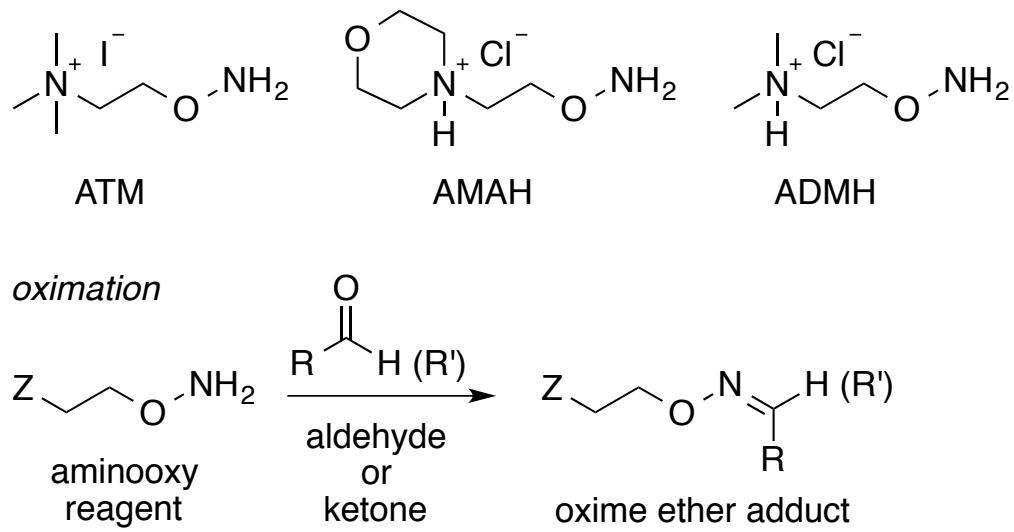

Scheme 3.1. Quaternary ammonium aminooxy reagents and oximation of aldehydes or ketones ( $\mathrm{Z}=$ ammonium moiety; $\mathrm{R}, \mathrm{R}^{\prime}=$ alkyl group).

\subsection{RESULTS AND DISCUSSION}

The synthesis 2-(aminooxy)- $N, N$-dimethylethan-1-aminium chloride (ADMH) (3) as depicted in scheme (Scheme 3.2) was accomplished in three steps: (1) reaction of ethanolamine (5) with $N$-hydroxyphthalimide (NHP) under standard Mitsunobu conditions (equimolar amounts of $\mathrm{NHP} / \mathrm{PPh}_{3} / \mathrm{DIAD}$ ) to obtain phthaloyloxy amine $\mathbf{6}$ in good yield; (2) hydrazinolyis by treatment of 6 with methylhydrazine at $0{ }^{\circ} \mathrm{C}$ in dry dichloromethane followed by Kugelrohr distillation of the liberated aminooxy product; and (3) acidification using aqueous hydrochloric acid under forcing conditions to hydrolyze atmospheric oxime ether adducts formed during handling of the Kugelrohr distillate. Recrystallization of the crude salt from isopropyl alcohol afforded ADMH as a white solid. 


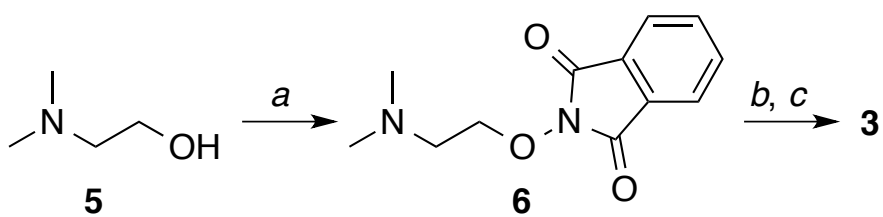

Scheme 3.2. Synthesis of ADMH. Reagents and conditions: $a$. $N$ hydroxyphthalimide, $\mathrm{PPh}_{3}$, DIAD, THF, $0{ }^{\circ} \mathrm{C}$ to rt, $12 \mathrm{~h} ; b . \mathrm{CH}_{3} \mathrm{NHNH}_{2}, \mathrm{CH}_{2} \mathrm{Cl}_{2}, 0{ }^{\circ} \mathrm{C}, 4.5 \mathrm{~h}$; c. $6 \mathrm{M} \mathrm{HCl}$, reflux, 22h.

The reaction kinetics of aminooxy reagent reacting with carbonyl compounds is initially assumed to be second order, irreversible reaction. The reaction rate is given by the following equation for the same molar amount of aminooxy reagent and carbonyl compound:

$r=\frac{d C_{\text {carbonyl }}}{d t}=-k C_{\text {Aminooxy }} C_{\text {carbonyl }}=-k C_{\text {carbonyl }}^{2}$

where $\mathrm{k}$ is the specific reaction rate, $\mathrm{C}$ is the concentration of the reactants, and $\mathrm{t}$ is the reaction time. The following equation can be obtained by integration of equation (1) from time zero to $t$ for the concentration of carbonyl compounds from initial $\mathrm{C}_{\mathrm{o}}$ to $\mathrm{C}(\mathrm{t})$

$\frac{1}{C(t)}-\frac{1}{C_{0}}=k t$

In order to verify that the reaction is irreversible elementary second order reaction for both reactants, plots of $1 / \mathrm{C}$ vs. time were made for all reactions. Figure 3.1 shows a representative plot $1 / \mathrm{C}$ vs. $\mathrm{t}$ for $\mathrm{AMAH}$ reacted with acetone at $-21,0$, and $21{ }^{\circ} \mathrm{C}$. Fig. 3.2 shows time dependent FT-ICR-MS spectra of AMAH-acetone. Figure 3.3 shows the plot of $1 / \mathrm{C}$ vs. $\mathrm{t}$ for $\mathrm{ADMH}$ reacted with acrolein at $-21,0$, and $21{ }^{\circ} \mathrm{C}$. Figure. 3.4 shows time dependent FT-ICR-MS spectra of ADMH-acetone. The linear plots with high $\mathrm{R}^{2}$ were obtained for all three aminooxy compounds reacted with carbonyl compounds. Therefore, the oximations reactions were found to be irreversible second order reactions. The 
corresponding values of the specific reaction rate $\mathrm{k}$ were obtained from the slope of the linear plot of $1 / \mathrm{C}$ vs. $\mathrm{t}$.
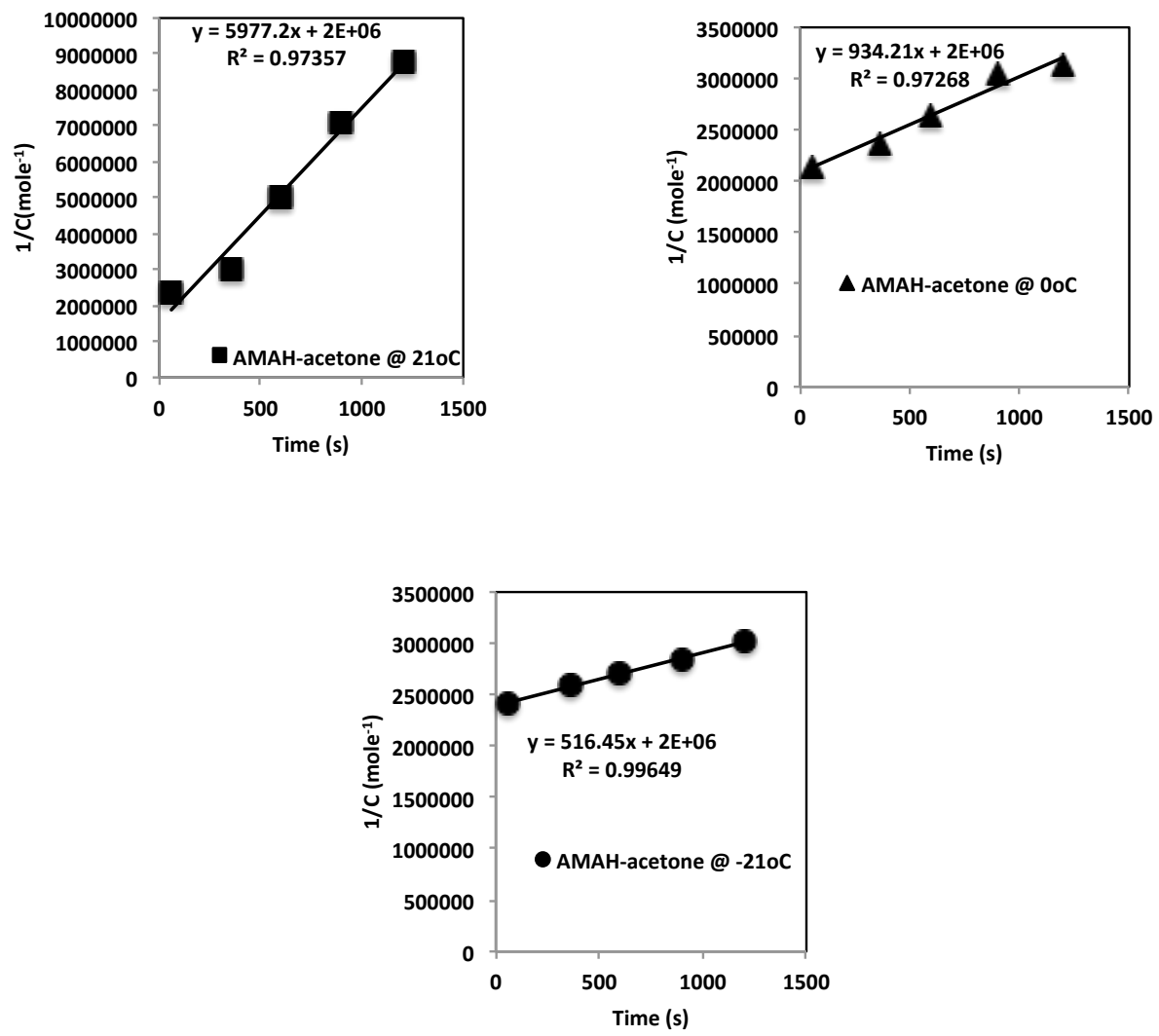

Figure 3.1. The plot of $1 / \mathrm{C}$ against time for oximation reaction between AMAH and acetone at $-21^{\circ} \mathrm{C}, 0{ }^{\circ} \mathrm{C}, 21^{\circ} \mathrm{C}$. 


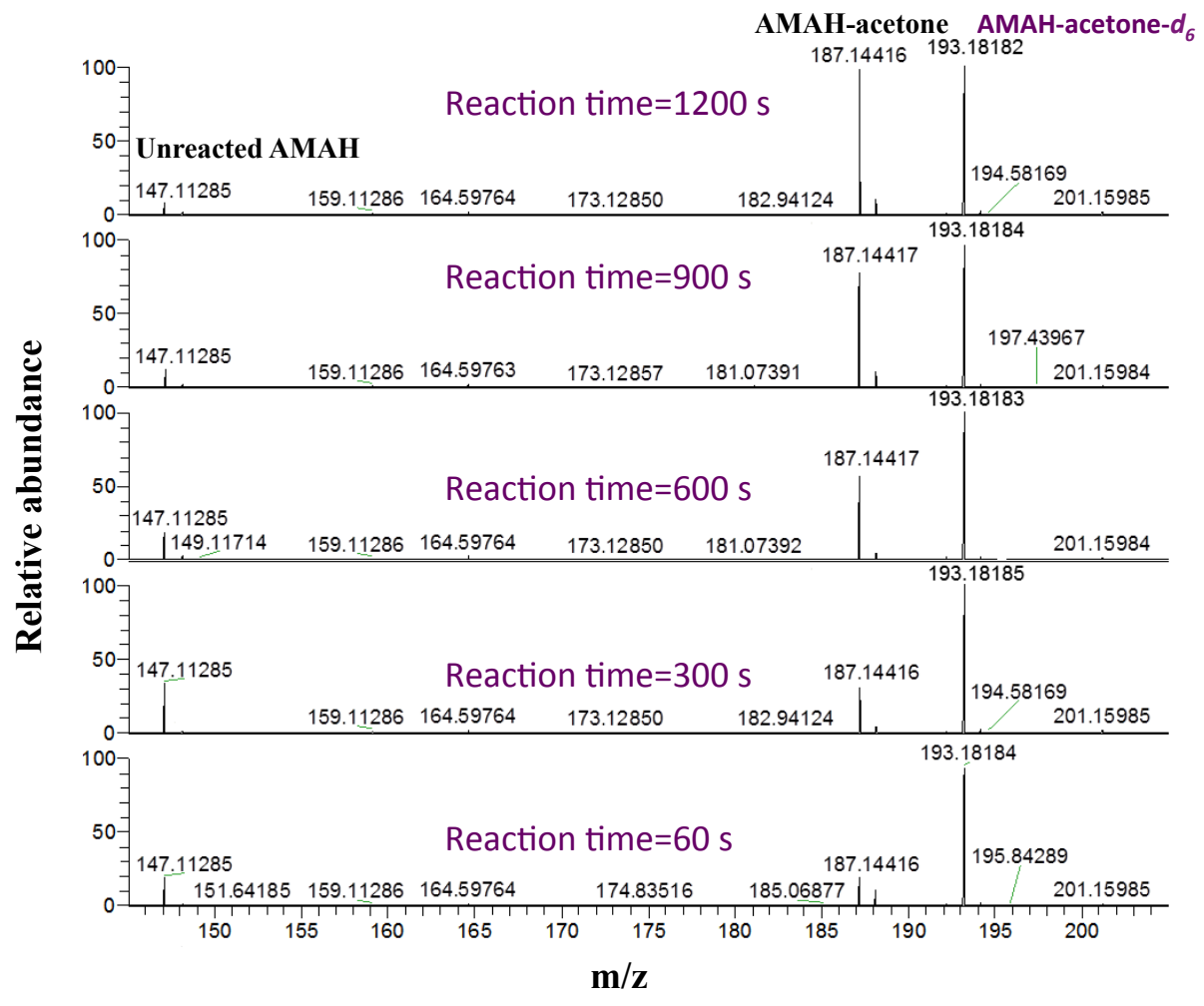

Figure 3.2. FT-ICR-MS Spectra overlay of oximation reaction between AMAH and acetone at $21{ }^{\circ} \mathrm{C}$ at times $\mathrm{t}=60,30,600,900$, and 1200 seconds corresponding with figure 3.2 .
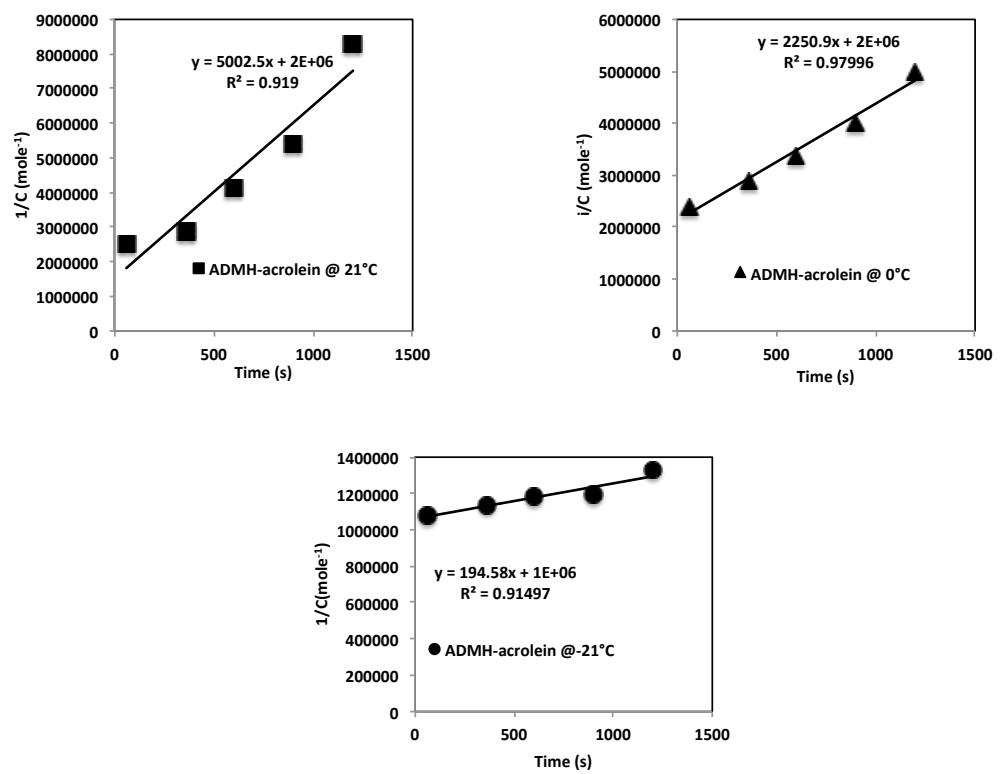

Figure 3.3. The plot of $1 / \mathrm{C}$ against time for oximation reaction between $\mathrm{ADMH}$ and acetone at $-21^{\circ} \mathrm{C}, 0{ }^{\circ} \mathrm{C}, 21^{\circ} \mathrm{C}$. 


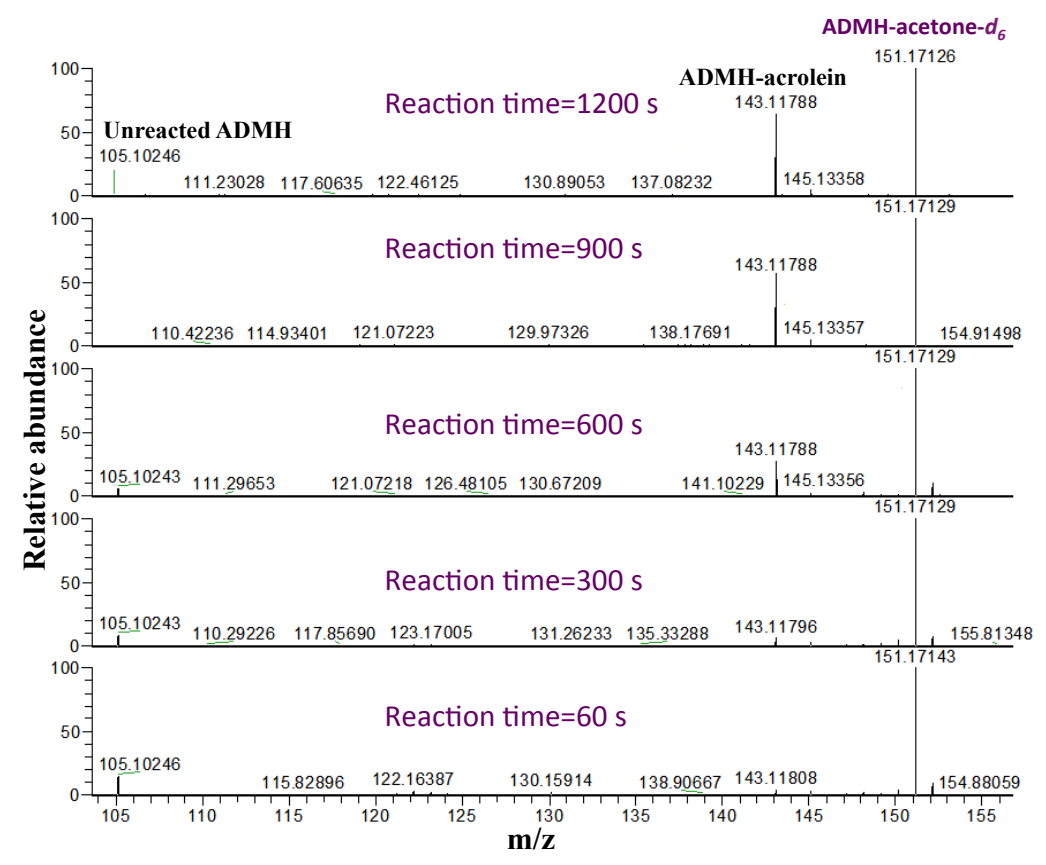

Figure 3.4. Spectra overlay of oximation reaction between $\mathrm{ADMH}$ and acrolein at $21{ }^{\circ} \mathrm{C}$ at times $\mathrm{t}=60,30,600,900,1200$ seconds corresponding with figure 3.4.

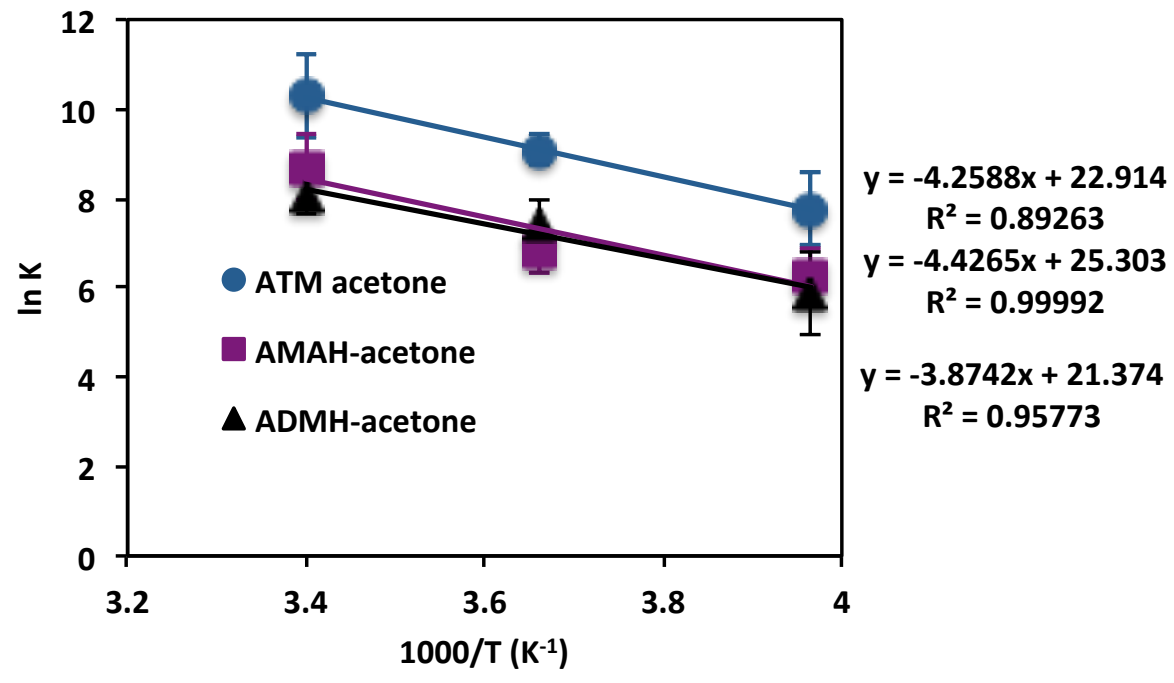

Figure 3.5. The graph of the natural logarithms of the reaction rate constants of oximation reaction as a function of the reciprocal of temperature between ADMH, AMAH, ATM and acetone. 
After the specific reaction factor $\mathrm{k}$ was determined for at least three different temperatures, the activation energy and frequency factor were determined from the Arrhenius equation,

$$
\ln k=-\left(\frac{E_{a}}{R}\right) \cdot\left(\frac{1}{T}\right)+\operatorname{lnk}_{0},
$$

the activation energy $E_{a}$ and frequency factor $k_{o}$ were determined from linear regression of the plot of the natural logarithm of $k(\ln k)$ vs. the reciprocal of temperature $1 / T .{ }^{137}$ Figure. 3.5 shows the plots of $\ln \mathrm{k}$ vs. 1/T for ADMH, AMAH, ATM reaction with acetone. The specific reaction rate $\mathrm{k}_{\mathrm{o}}$ of ATM reaction with acetone is higher than that of AMAH and ADMH reaction with acetone.

Figures. 3.6 to 3.10 show the linear regression plots of the natural logarithm of reaction constant ln $\mathrm{k}$ between ADMH, AMAH and ATM and carbonyl compounds. These plots are all linear with negative slope from which the activation energies of the oxime ethers were computed. Table 3.1 shows the activation energy Ea and frequency factor $\mathrm{k}_{\mathrm{o}}$ for the reactions ADMH, AMAH and ATM with different aldehydes and ketones including $\alpha, \beta$-unsaturated aldehydes, acrolein and crotonaldehyde. Generally, activation energies of ADMH are lowest of all the three aminoxies reacting with all the carbonyl compounds followed by AMAH. We suspect that this could be because it is the smallest of the three molecules. The smaller the molecule, the faster it moves and the higher kinetic energy, which results in collision and formation of products. AMAH is however bulkier than ATM but has smaller activation energy and this can be attributed to the presence of titratable acidic proton which can catalyze the reaction by activating the carbonyl carbon of the aldehydes and ketones. The Ea are also seen to increase with increasing carbon number of the ketones. The Ea of acetone is higher than propanal even 
though they both have 3 carbon atoms because aldehydes are more reactive than ketones. The $\mathrm{k}_{\mathrm{o}}$, which shows the frequency of collision increase down the table for all the carbonyl compounds except for MIBK with some branching. This behavior can also be attributed to the presence of titratable acidic proton, which catalyzes the reaction by lowering the activation energy. Because of low activation energies of ADMH and AMAH reactions with carbonyls, the contribution of $k_{0}$ to the specific reaction rate $\mathrm{k}$ is significant. Therefore, even if the activation energy for ADMH and AMAH reaction with carbonyls increase, the increase of $k_{0}$ for this reaction results in higher specific reaction rate.

Figure. 3.7 and Figure 3.8 show the plots of $\ln \mathrm{k}$ vs. 1/T for ADMH, AMAH, ATM reaction with acrolein and crotonaldehyde, the $\alpha, \beta$-unsaturated species. The activation energy Ea of the three aminooxies are generally higher than those of the saturated aldehyde and ketones because of the $\pi$ electron density of the double bond, which slows down the nucleophilic attack by the aminooxy reagent on the carbonyl carbon. Also the energy of activation of ADMH and AMAH with these $\alpha, \beta$-unsaturated aldehydes are closer and lower than that of ATM. They are therefore more reactive towards acrolein and crotonaldehyde than ATM. The higher frequency factor $k_{\mathrm{o}}$ for the reactions of ATM with acrolein and crotonaldehyde did not necessarily translate to higher reactivity. In fact, the Ea of ATM with crotonaldehyde is 1.5 times higher than that of ADMH. 


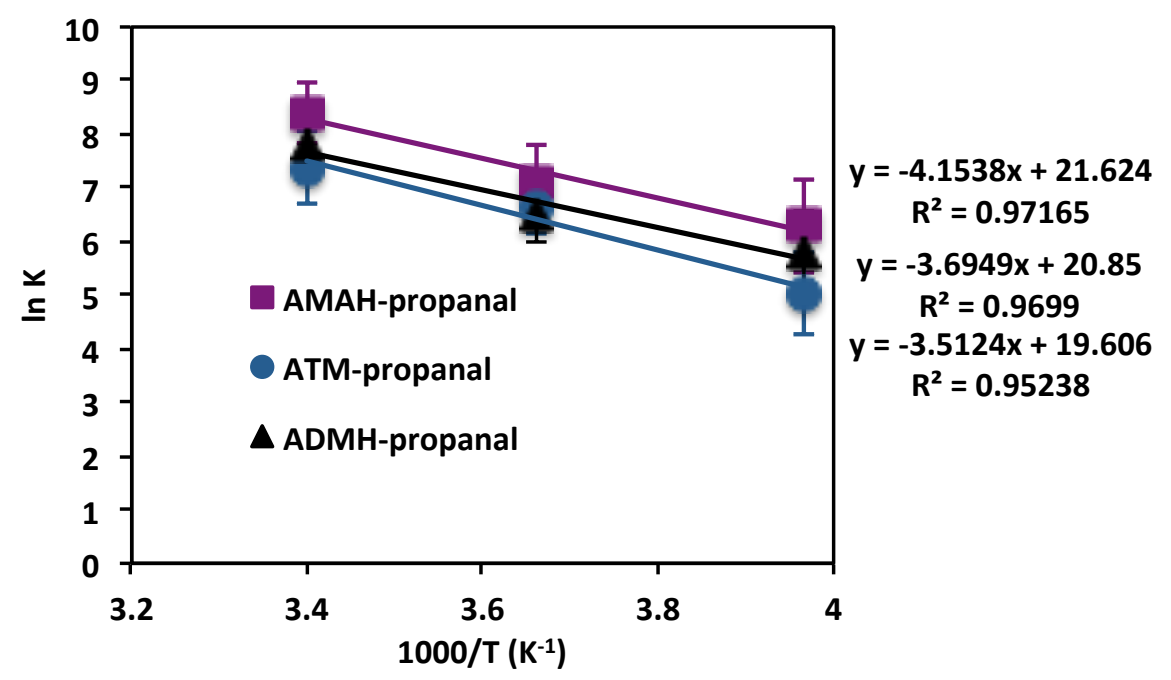

Figure 3.6. The graph of the natural logarithms of the rate constants of oximation reaction as a function of the reciprocal of temperature between ADMH, AMAH, ATM and propanal.

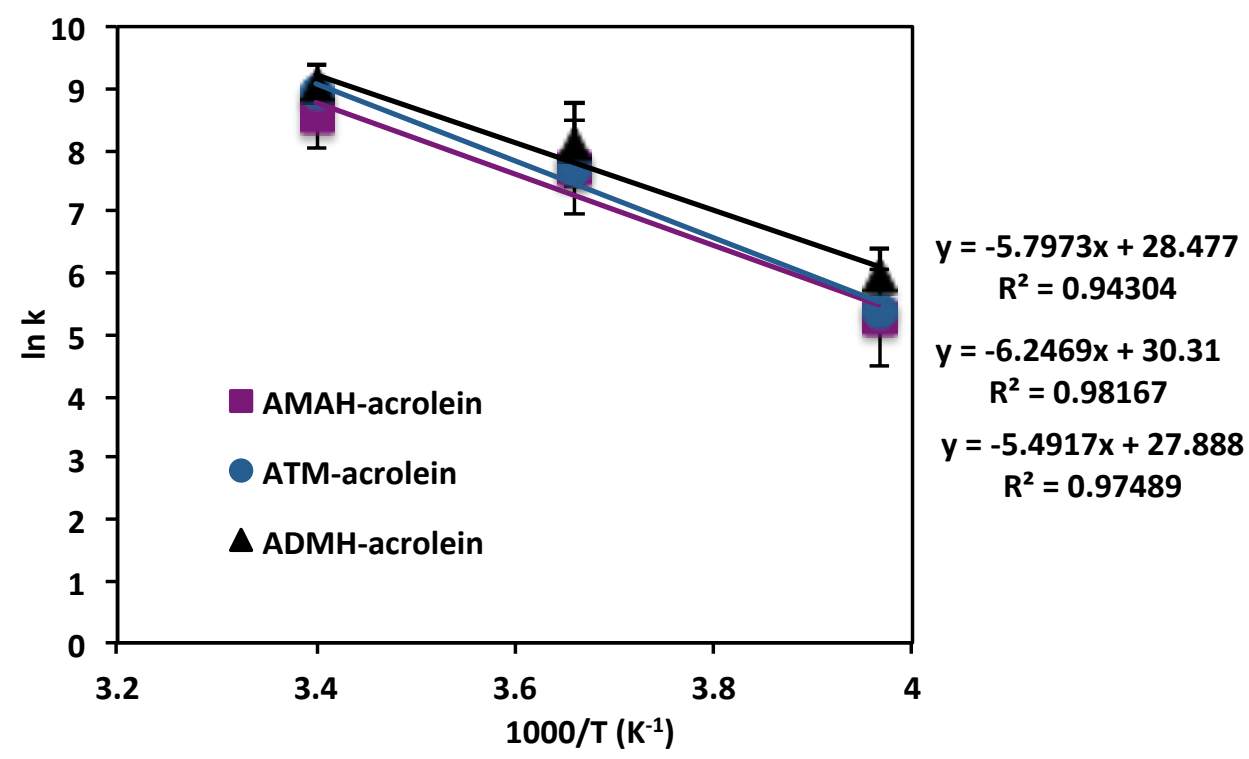

Figure 3.7. The graph of the dependence of the natural logarithms of the rate constants of oximation reaction as a function of the reciprocal of temperature between ADMH, AMAH, ATM and acrolein. 


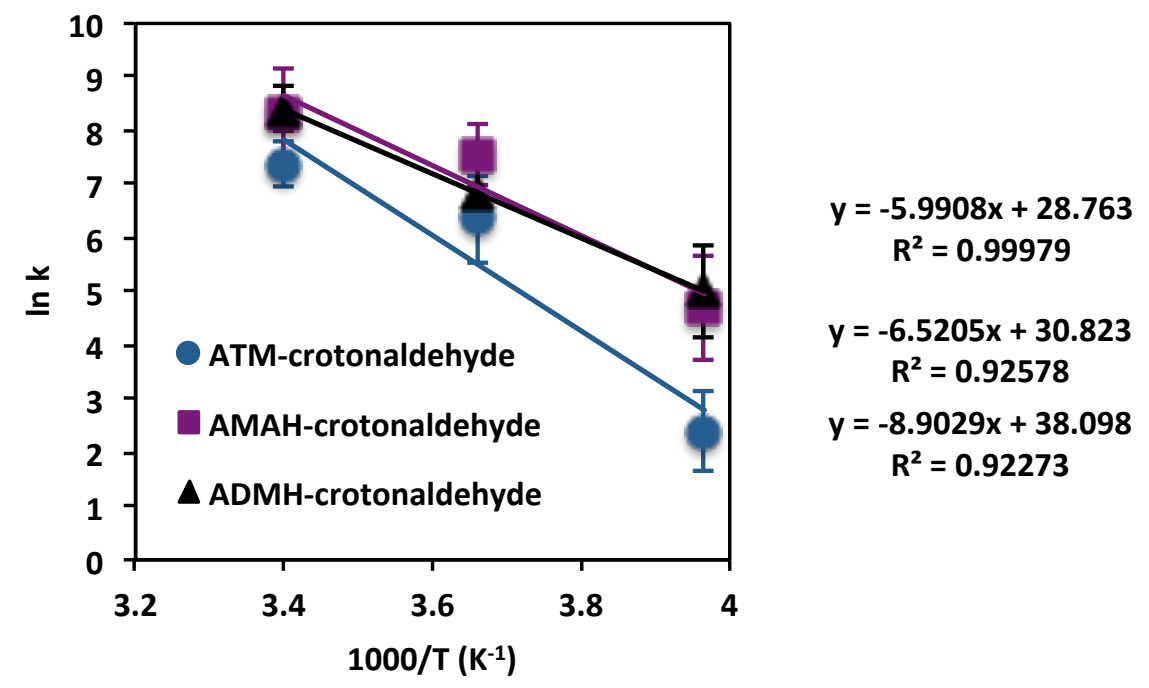

Figure 3.8. The graph of the dependence of the natural logarithms of the rate constants of oximation reaction as a function of the reciprocal of temperature between ADMH, AMAH, ATM and crotonaldehyde.

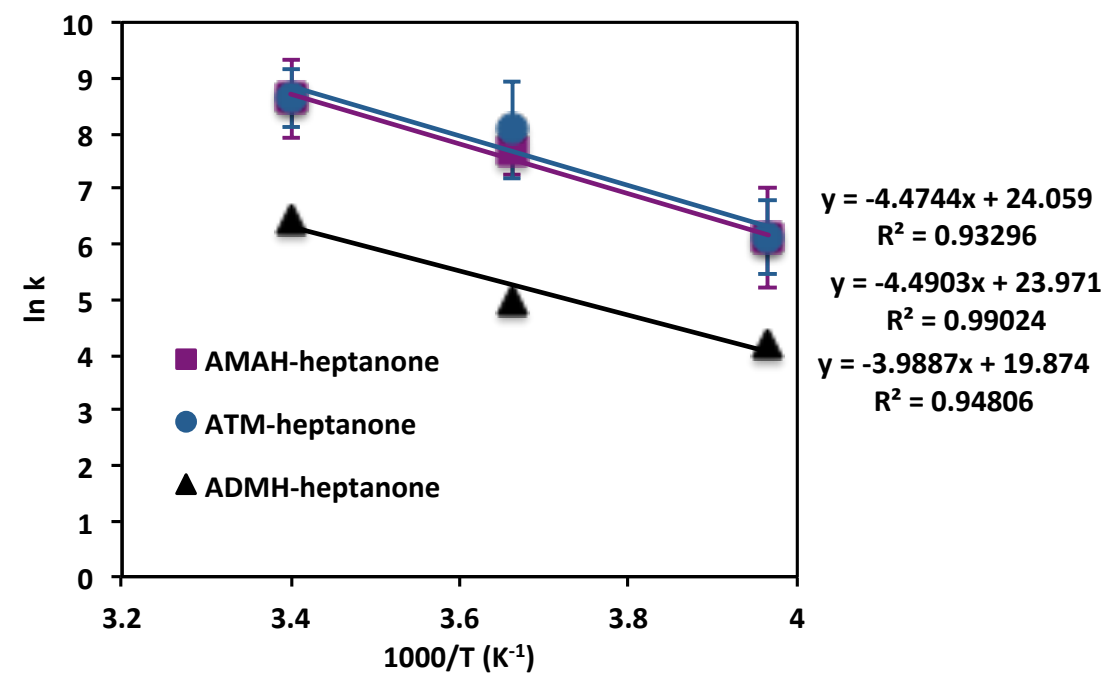

Figure 3.9. The graph of the dependence of the natural logarithms of the rate constants of oximation reaction as a function of the reciprocal of temperature between ADMH, AMAH, ATM and 2-heptanone. 


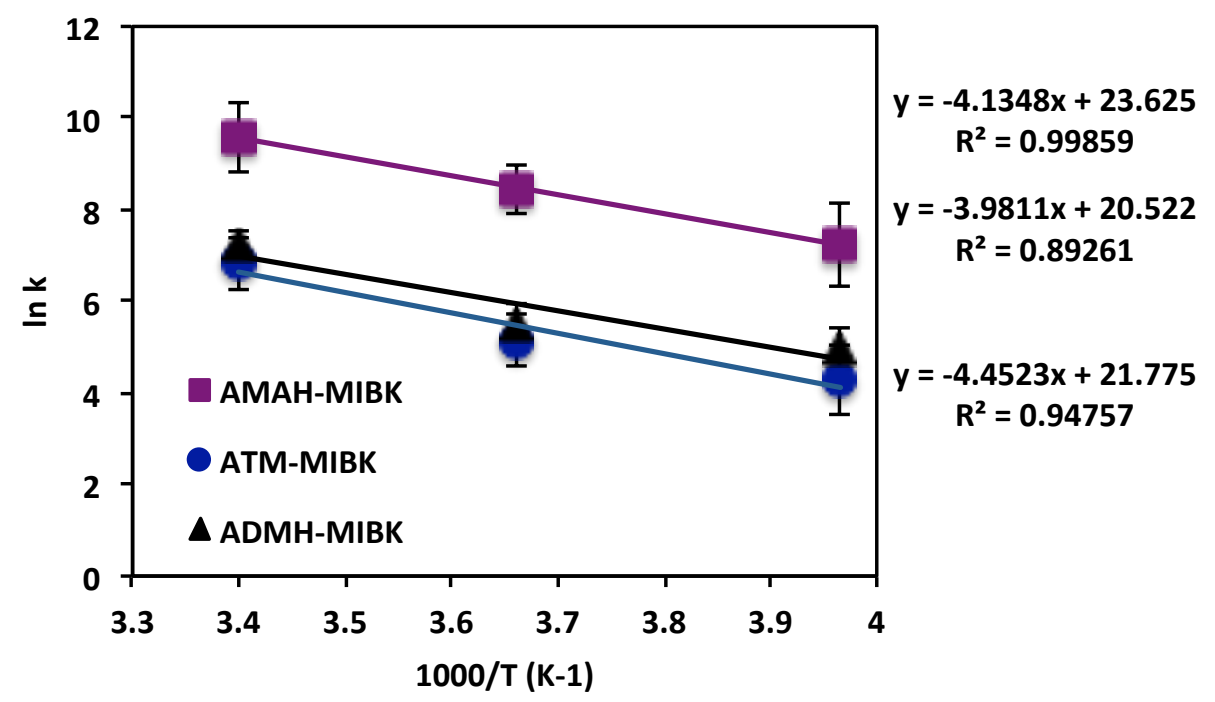

Figure 3.10. The graph of the dependence of the natural logarithms of the rate constants of oximation reaction as a function of the reciprocal of temperature between ADMH, AMAH, ATM and methyl isobutyl ketone (MIBK).

Table 3.1. The Activation Energies and frequency factor of AMAH, ADMH, and ATM reactions with acetone (C3), propanal (C3), 2-heptanone (C7), MIBK (C6) acrolein (C3) and crotonaldehyde (C4).

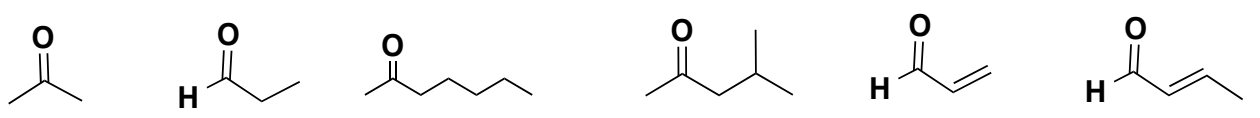

\begin{tabular}{|l|llllll|}
\hline ADMH & \multicolumn{5}{|c|}{} \\
$\mathrm{E}_{\mathrm{a}}\left(\mathrm{kJmol}^{-1}\right)$ & 32.21 & 29.20 & 33.16 & 33.1 & 45.66 & 49.81 \\
$\mathrm{~K}_{\mathrm{o}}\left(\mathrm{s}^{-1}\right)$ & $1.92 \times 10^{9}$ & $3.27 \times 10^{8}$ & $4.28 \times 10^{8}$ & $8.18 \times 10^{8}$ & $1.29 \times 10^{12}$ & $3.10 \times 10^{12}$ \\
\hline $\mathrm{AMAH}$ & & & & & \\
$\mathrm{E}_{\mathrm{a}}\left(\mathrm{kJmol}^{-1}\right)$ & 34.30 & 34.54 & 37.33 & 34.38 & 48.2 & 54.21 \\
$\mathrm{~K}_{\mathrm{o}}\left(\mathrm{s}^{-1}\right)$ & $8.94 \times 10^{9}$ & $1.14 \times 10^{9}$ & $2.57 \times 10^{10}$ & $1.82 \mathrm{E} \times 10^{10}$ & $2.33 \mathrm{Ex} 10^{12}$ & $2.43 \times 10^{13}$ \\
\hline $\mathrm{ATM}$ & & & & & \\
$\mathrm{E}_{\mathrm{a}}\left(\mathrm{kJmol}^{-1}\right)$ & 36.80 & 30.72 & 37.20 & 37.02 & 51.94 & 74.02 \\
$\mathrm{~K}_{\mathrm{o}}\left(\mathrm{s}^{-1}\right)$ & $9.75 \times 10^{10}$ & $2.46 \mathrm{E} \times 10^{9}$ & $2.81 \times 10^{10}$ & $2.86 \times 10^{9}$ & $1.46 \times 10^{13}$ & $3.51 \times 10^{16}$ \\
\hline
\end{tabular}




\subsection{HYDRAZINE SYNTHESIS}

We proposed to synthesize 2-hydrazinyl- $N, N, N$-trimethylethan-1-ammonium iodide abbreviated HTM (Figure 3.11) because it is expected to be more nucleophilic and reactive towards carbonyl compounds than ATM. The reactivities of the two reagents (HTM and HTM) were also studied and compared.

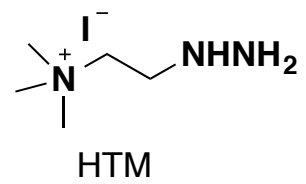

Figure 3.11. 2-Hydrazinyl- $N, N, N$ - trimethylethan-1-ammonium iodide (HTM).

\subsubsection{Synthetic Route to HTM}

The synthesis of 2-hydrazinyl- $N, N, N$-trimethylethan-1-ammonium iodide (HTM) is depicted in Scheme 3.3 and was accomplished in three steps beginning with quaternization of commercially available $2-(N, N$-dimethylamino) ethanol with methyl iodide. ${ }^{138-139}$ The resultant quaternary ammonium alcohol $\mathbf{2}$ was converted to chloride $\mathbf{3}$ by refluxing in excess thionyl chloride. Figures 3.12 and 3.13 show ${ }^{1} \mathrm{H}$ NMR spectra of the alcohol 2 and chloride 3 that highlight the disappearance of hydroxyl proton (triplet, $\delta$ 5.25) as well as the downfield shifts in the methylene proton of the chloride 3 . The product formation was also confirmed by high-resolution mass spectrometry (Figure 3.14). Hydrazine functionality was installed by refluxing chloride 3 in a solution of excess hydrazine monohydrate in tetrahydrofuran. Excess hydrazine monohydrate was distilled off and the residue crystallized from ethanol to give the pure HTM. Figures 3.15 and 3.16 show ${ }^{1} \mathrm{H}$ NMR and ${ }^{13} \mathrm{C}$ NMR of purified HTM. 


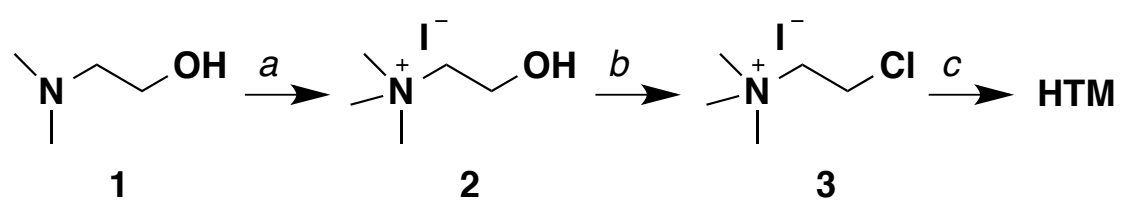

Scheme 3.3. Synthesis of HTM. Reagents and conditions: a. a. $\mathrm{CH}_{3} \mathrm{I}, \mathrm{CH}_{2} \mathrm{Cl}_{2}$, sealed tube, $50{ }^{\circ} \mathrm{C}, 12 \mathrm{~h}, 90 \%$; b. $\mathrm{SOCl}_{2}, 80{ }^{\circ} \mathrm{C}, 4$ h, $98 \%$; c. $\mathrm{NH}_{2} \mathrm{NH}_{2} \cdot \mathrm{H}_{2} \mathrm{O}$ (xs), THF, reflux, 6 h, $43 \%$.

It is noteworthy that the FT-ICR-MS spectrum of pure HTM showed that this compound complexes with iodide to form a dimer, but the dimer readily reacts with acetone $d_{6}$ to form the corresponding hydrazone adduct (Figure 3.17). This formation of dimer complex with iodine and a silver nitrate test that gave a pale yellow coloration confirmed that indeed the counter-ion of the HTM is iodide.

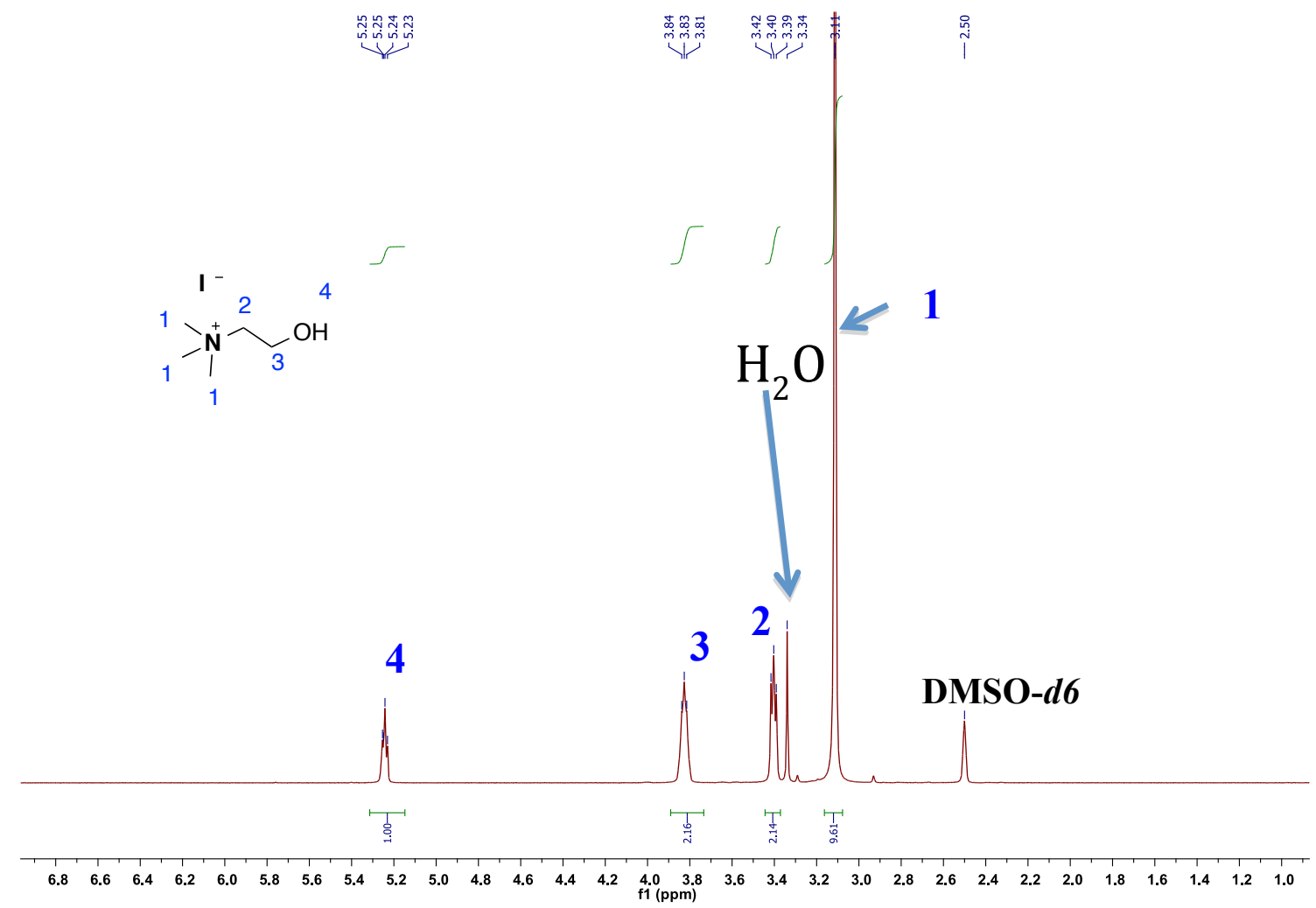

Figure 3.12. The ${ }^{1} \mathrm{H}$ NMR spectrum of alcohol 2 in DMSO- $d_{6}$. 


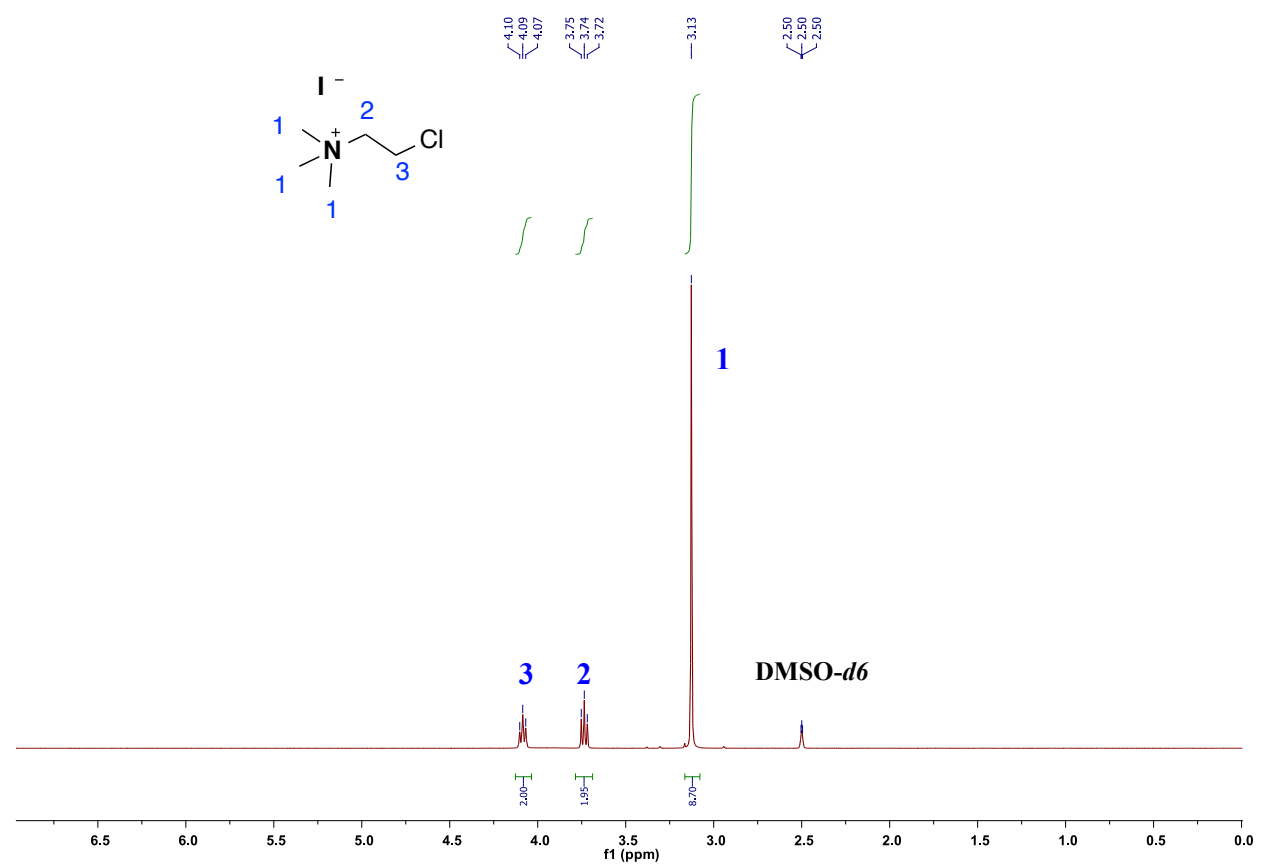

Figure 3.13. The ${ }^{1} \mathrm{H}$ NMR spectrum of chloride 3 in DMSO- $d_{6}$.

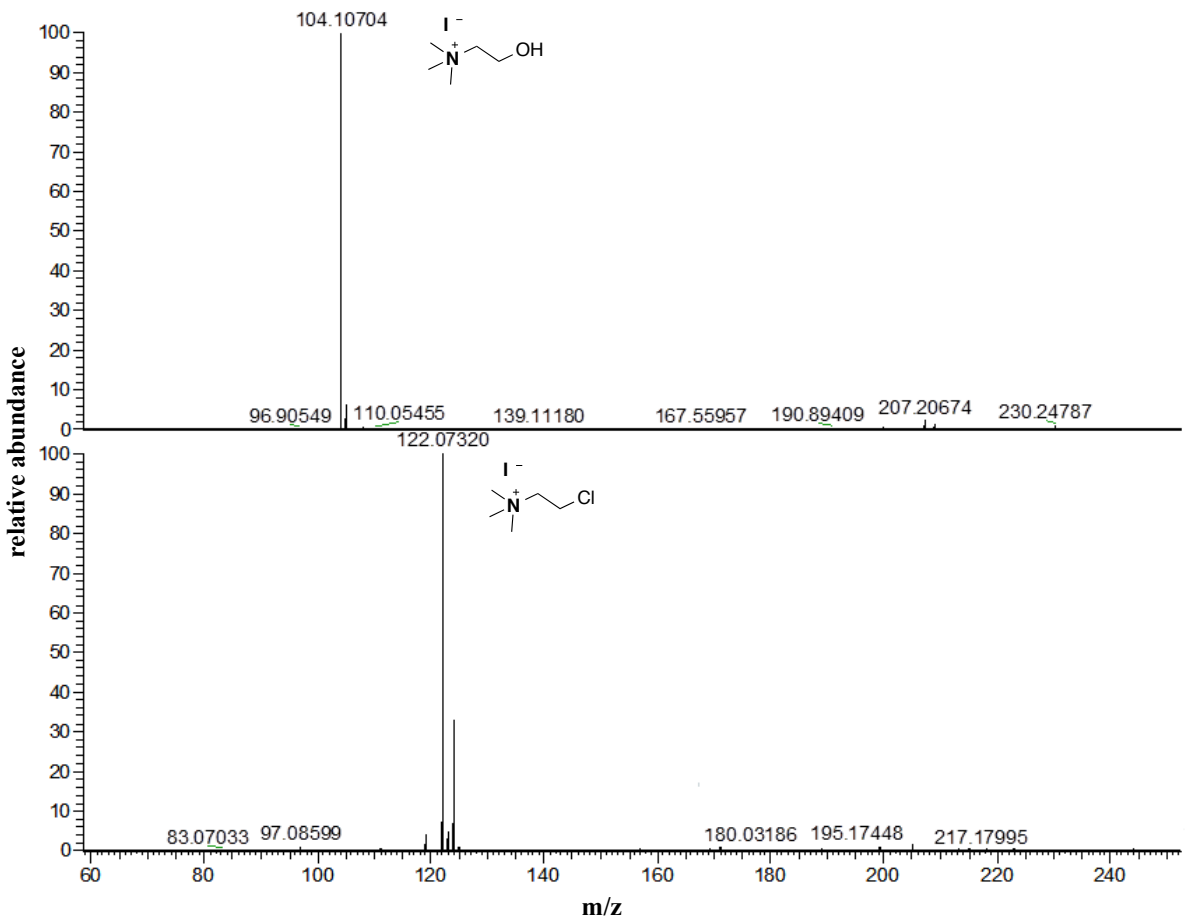

Figure 3.14. The high-resolution mass spectrometry spectra overlay of alcohol 2 and chloride 3. 


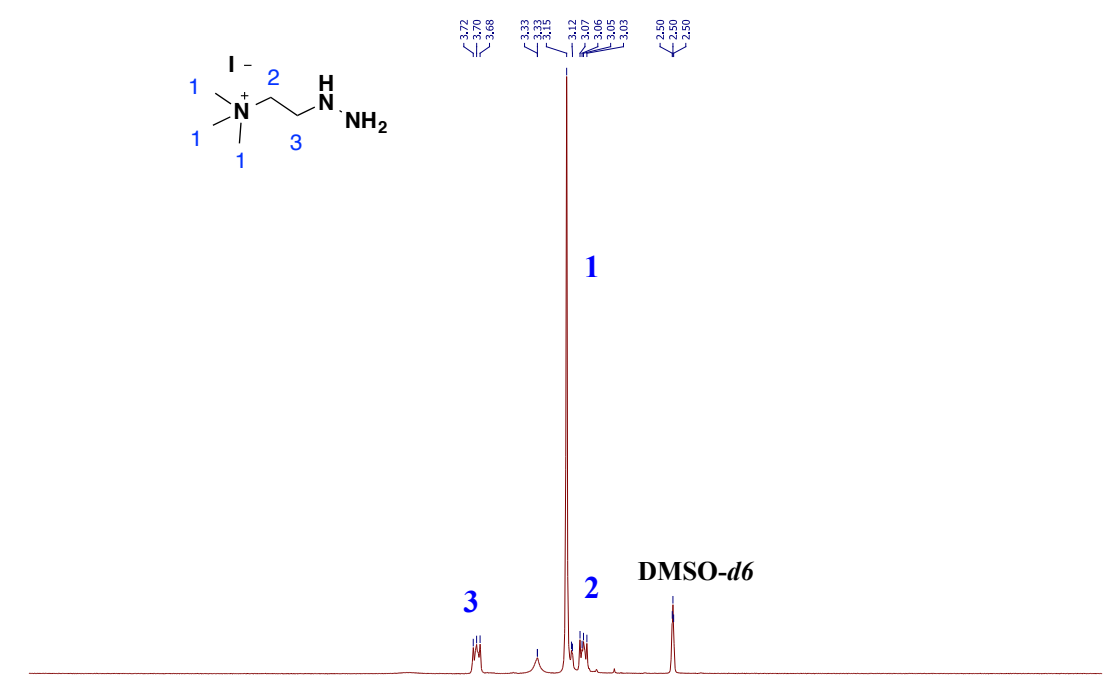

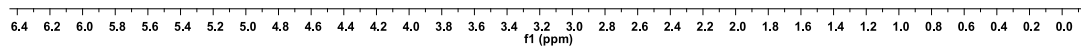

Figure 3.15. The ${ }^{1} \mathrm{H}$ NMR spectrum of HTM in DMSO- $d_{6}$

\section{ชำ}
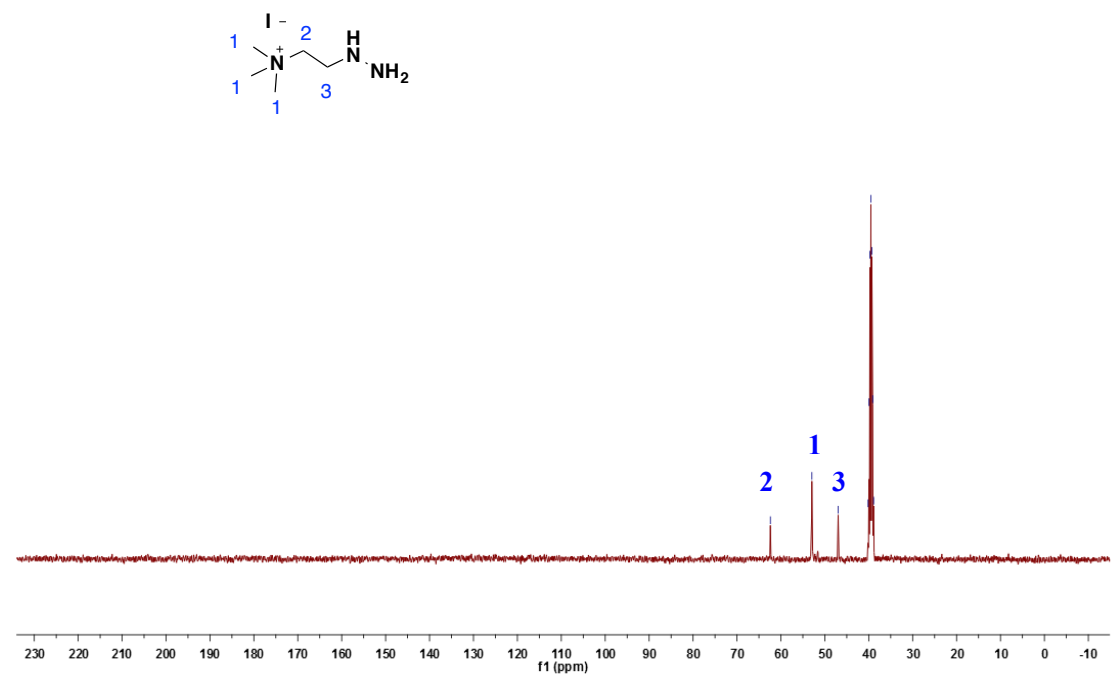

Figure 3.16. The ${ }^{13} \mathrm{C}$ NMR spectrum of HTM in DMSO- $d_{6}$. 


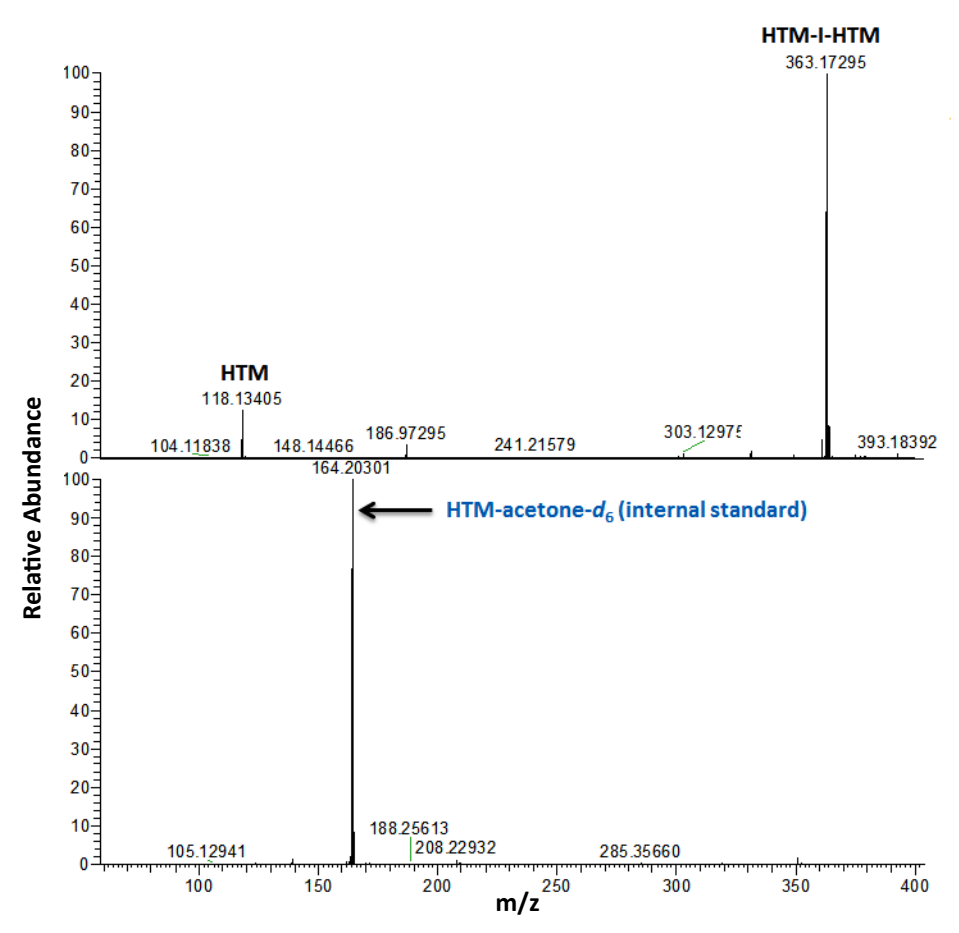

Figure 3.17. The FT-ICR-MS of the HTM and HTM-acetone- $d_{6}$ adduct (internal standard). The FT-ICR-MS spectrum of HTM-acetone $\mathrm{d}_{6}$ (bottom) was acquired after the addition of an equimolar amount acetone $\mathrm{d}_{6}$ to HTM sample and allowing it to stand for 1 hour.

In order to compare the reactivity of the existing aminooxy reagent (ATM) with HTM, we performed a kinetic study of these reagents reacting with carbonyl compounds. We selected two saturated and two unsaturated carbonyl compounds: propanal, acrolein, acetone and crotonaldehyde. The reaction kinetics of HTM and ATM with these carbonyl compounds was studied using FT-ICR-MS. The reaction kinetics of aminooxy and hydrazine reagents reacting with carbonyl compounds is initially assumed to be an elementary second order, irreversible reaction. By applying equation 2, a plot of 1/C against $\mathrm{t}$ gave straight-line graphs from where k values were obtained. Figure 3.18 shows a plot of $1 / \mathrm{C}$ against $\mathrm{t}$ for the reaction between $\mathrm{HTM}$ and acrolein at $-21{ }^{\circ} \mathrm{C}, 0{ }^{\circ} \mathrm{C}$, and 21 ${ }^{\circ} \mathrm{C}$. Also, application of Arrhenius equation 3 and a plot of $\ln \mathrm{k}$ against $1 / \mathrm{T}$ afforded straight-line graphs with negative slope from where the activation energies for selected 
carbonyl compounds were computed. Figure 3.19 shows the plot of $\ln \mathrm{k}$ against $1 / \mathrm{T}$ for HTM-propanal, HTM-acetone, HTM-acrolein and HTM-crotonaldehyde while figure 3.20 shows the plot of $\ln \mathrm{k}$ against 1/T for ATM-propanal, ATM-acetone, ATM-acrolein and ATM-crotonaldehyde.

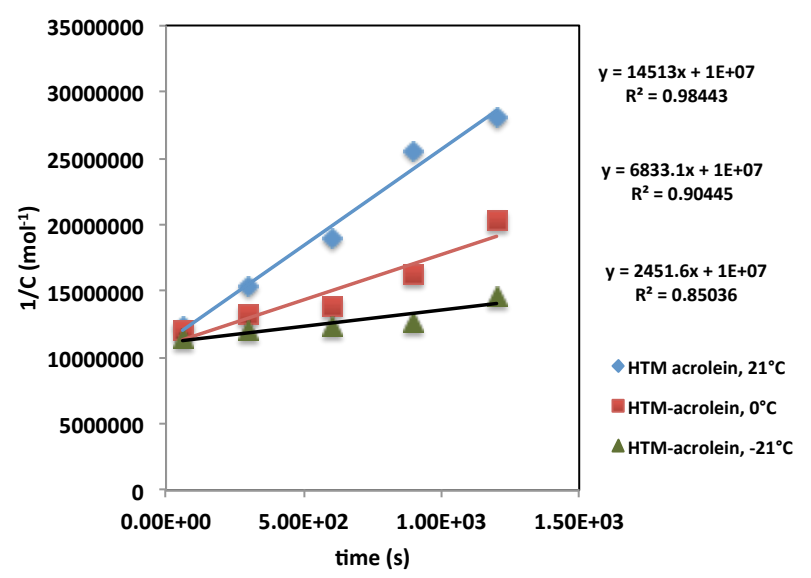

Figure 3.18. The graph of $1 / \mathrm{C}$ against time for hydrazone formation reaction between HTM and acrolein at $-21^{\circ} \mathrm{C}, 0{ }^{\circ} \mathrm{C}$, and $21^{\circ} \mathrm{C}$.

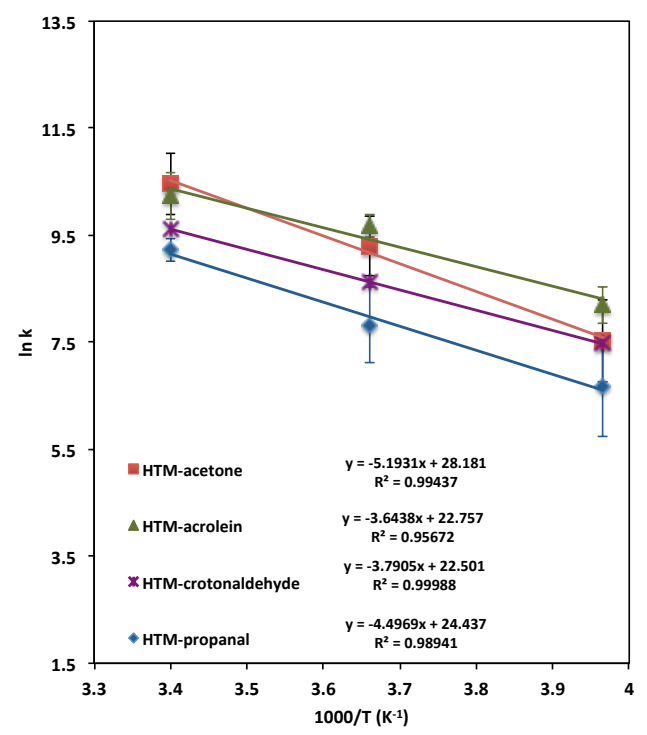

Figure 3.19. The graph of the dependence of the natural logarithms of the rate constants of hydrazone formation reaction as a function of the reciprocal of temperature between HTM and propanal, acetone, acrolein, and crotonaldehyde. 


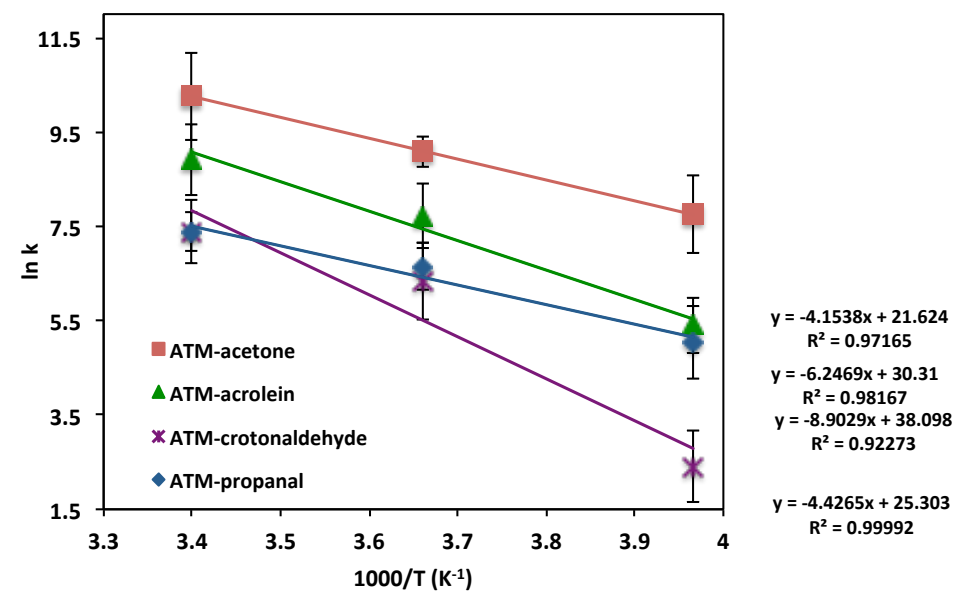

Figure 3.20. The graph of the dependence of the natural logarithms of the rate constants of hydrazone formation reaction as a function of the reciprocal of temperature between ATM and propanal, acetone, acrolein, and crotonaldehyde.

The activation energies for reactions of HTM with volatile ketones and aldehydes were compared to those measured for ATM - the aminooxy analogue. The results showed that HTM has lower activation energy for $\alpha, \beta$-unsaturated compounds than ATM implying that HTM reacts the faster with the unsaturated substrates because the energy barrier that must be overcome for the reaction to occur is low. On the other hand, the aminooxy reagents have lower activation energies for saturated carbonyl compounds thereby reacting faster with saturated carbonyl substrates than HTM (Table 3.2). 
Table 3.2. Activation energies of HTM and ATM adducts of selected carbonyl compounds.

\begin{tabular}{|ll|l|l|}
\hline Entry & Carbonyl & $\begin{array}{l}\text { Ea } \\
\text { HTM } \\
\text { adduct }\end{array}$ & $\begin{array}{l}\text { Ea } \\
\text { ATM } \\
\text { adduct }\end{array}$ \\
\hline 1 & Propanal & 37.39 & 36.80 \\
\hline 2 & Acrolein & 30.30 & 51.96 \\
\hline 3 & Acetone & 43.18 & 34.54 \\
\hline 4 & Crotonaldehyde & 31.51 & 74.02 \\
\hline
\end{tabular}

$\mathrm{E}_{\mathrm{a}}$ in $\mathrm{KJmol}^{-1}$

\subsubsection{Capture efficiency}

To compare reactivities of aminooxy reagent ATM and hydrazine HTM with with $\alpha, \beta$-unsaturated aldehydes in a silicon microreactor, we determined the percentages of unsaturated carbonyl compounds that were captured by the HTM- and ATM-coated chips. Acrolein and crotonaldehyde were selected for this study. A known amount of acrolein or crotonaldehyde in methanol was injected into $1 \mathrm{~L}$ of air in a Tedlar bag. The loaded air sample then passed through an ATM- or HTM-coated chip (Figure 3.21). The percentage of the captured carbonyl was calculated as capture efficiency. Figure 3.22 shows the capture efficiency of HTM and ATM with acrolein and crotonaldehyde. The capture efficiency of HTM is about 2 times higher than ATM in trapping acrolein or crotonaldehyde. 


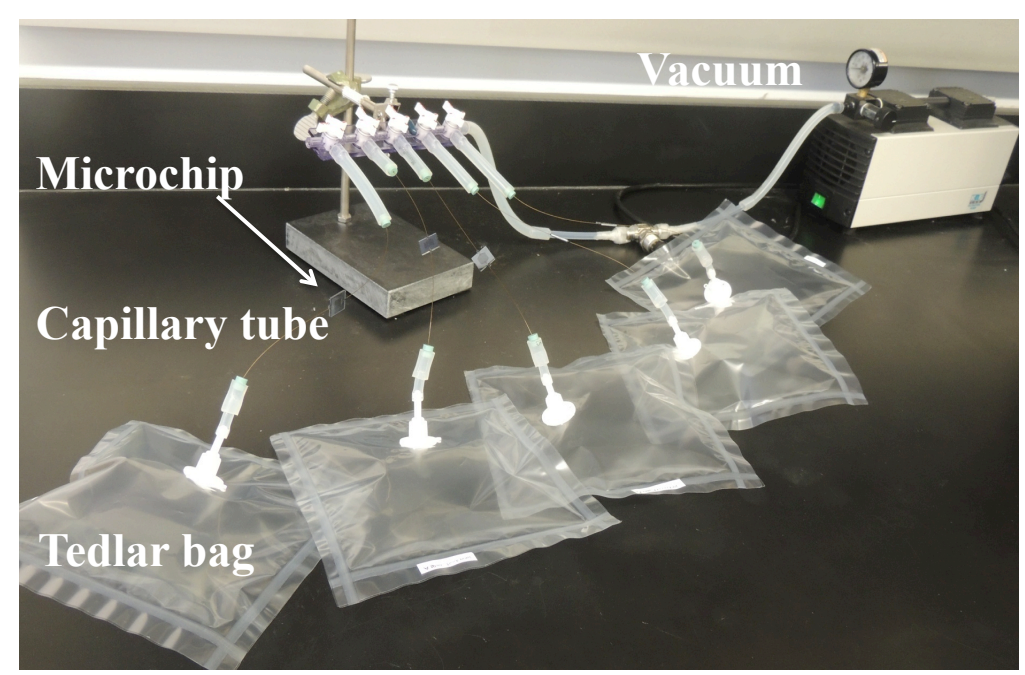

Figure 3.21. The experimental set-up showing the preconcentration of carbonyls in the microchip.

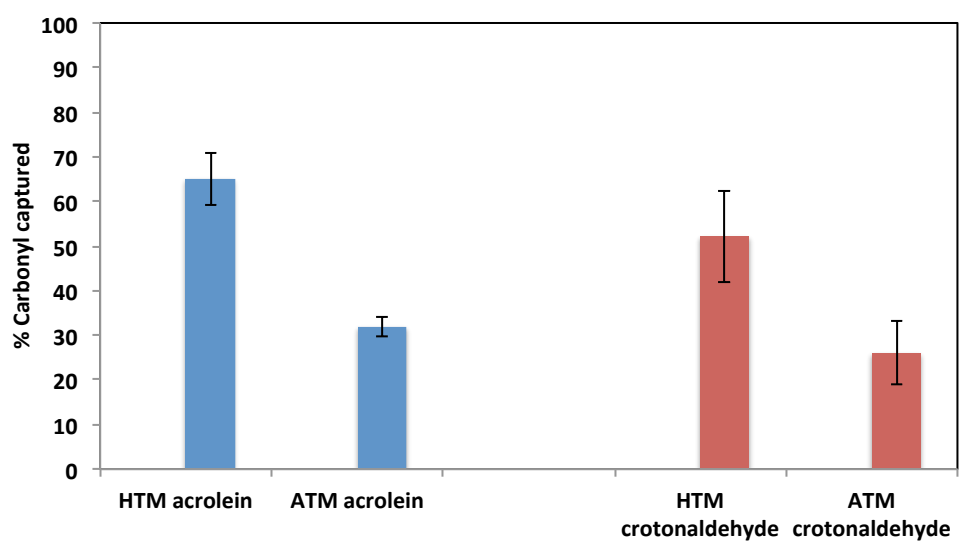

Figure 3.22. The capture efficiency graph of HTM and ATM with acrolein and crotonaldehyde. The graph shows that HTM captures the unsaturated carbonyls with efficiency twice as high as ATM.

\subsubsection{Cigarette smoke and exhaled breath analysis}

To derivatize of the carbonyl compounds generated in tobacco cigarette smoke, a Kentucky reference cigarette 3R4F smoke was collected using a $100 \mathrm{~mL}$ capacity syringe fitted with rubber tubing. $60 \mathrm{~mL}$ of smoke was collected per puff and a total of 9 puffs were collected into a 10-liter Tedlar bag. A $2 \mathrm{~mL}$ aliquot was taken out and injected into a $500 \mathrm{~mL}$ tedlar bag containing $498 \mathrm{~mL}$ of pure air for derivatization and analysis. 
In order to quantify carbonyl compounds, calibration curves were built using FTICR-MS by plotting the ratio of the relative abundance of carbonyl compounds to an internal reference $(y)$, against the mole ratio of the carbonyl compounds to the internal reference (x). HTM and ATM internal standards were prepared by reacting and equimolar amount deuterated acetone- $\mathrm{d}_{6}$ to HTM and ATM and left overnight to form HTM and ATM acetone- $\mathrm{d}_{6}$ adduct. $4 \mathrm{nmol}$ of the internal standard was added to each of the serially diluted HTM- or ATM-carbonyl compound adduct solution. The calibration curve showed an excellent linearity between the intensity ratio of carbonyl adduct to internal reference $\left(\mathrm{I}_{\text {Carbonyl }} / \mathrm{I}_{\mathrm{IR}}\right)$ and the molar ratio of carbonyl adduct to reference $\left(\mathrm{M}_{\text {Carbonyl }} / \mathrm{M}_{\mathrm{IR}}\right)$ with $0.02 \mathrm{nmol}$ to $7 \mathrm{nmol}$ dynamic range. The concentrations of detected carbonyl compounds were calculated from the calibration curves for different carbonyl adducts (Figures 3.23. and 3.24).

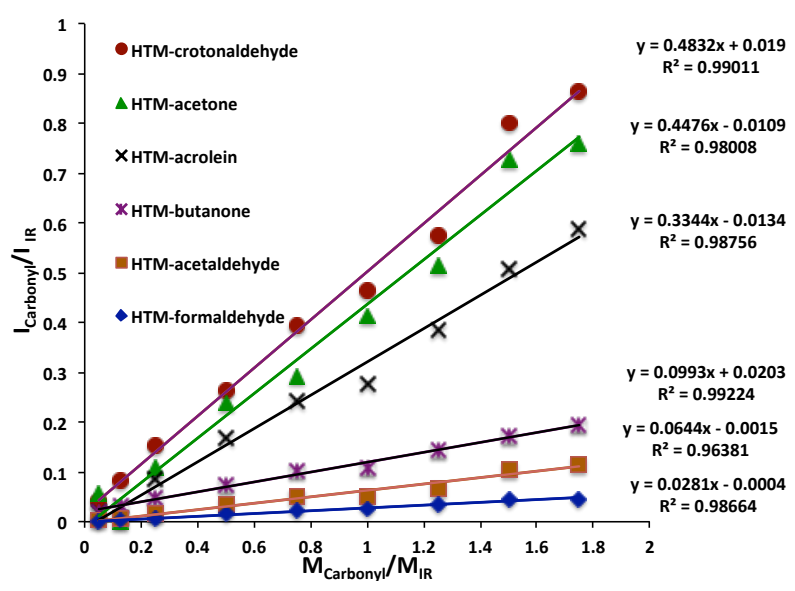

Figure 3.23. The calibration curves of HTM-carbonyl adducts using HTM-acetone- $d_{6}$ adduct as internal standard. 


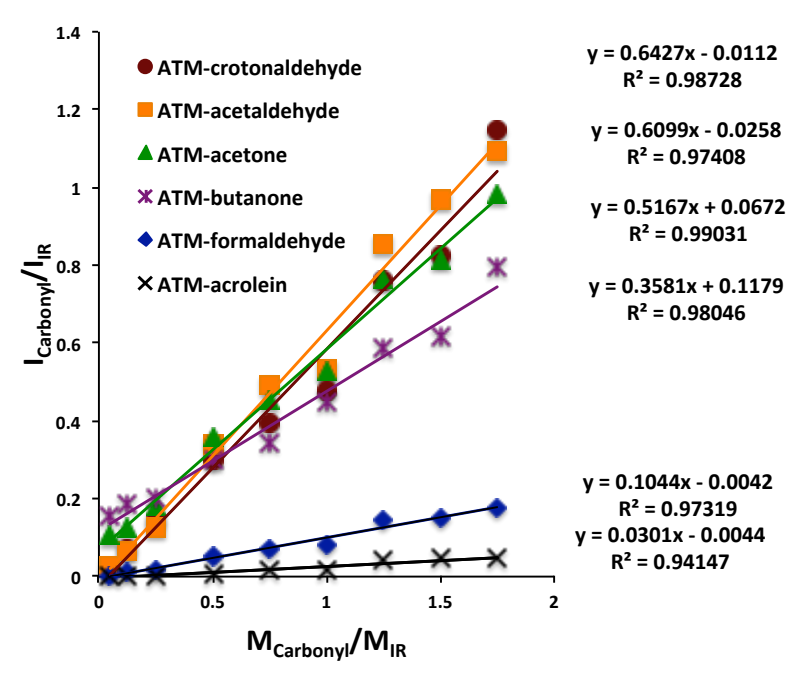

Figure 3.24. The calibration curves of ATM-carbonyl adducts using ATM-acetone- $d_{6}$ adduct as internal standard.

Table 3.3 shows the amounts of carbonyl compounds, in $\mu \mathrm{g} /$ cigarette, captured by HTM and ATM. The amount of unsaturated acrolein and crotonaldehyde derivatized by the HTM was higher than those of the ATM whereas the ATM captured more of the saturated aldehydes and ketones.

Table 3.3. ${ }^{140}$ Carbonyl compounds quantified from 3R4F cigarette smoke. ${ }^{\mathrm{a}, \mathrm{b}}$

\begin{tabular}{|l|llllll|}
\hline & Formaldehyde & Acetaldehyde & Acrolein & Acetone & 2-butanone & crotonaldehyde \\
\hline ATM & $\mathbf{9 0 . 5 0} \pm 6.18$ & $\mathbf{9 0 6 . 4 0} \pm 97.51$ & $\mathbf{2 9 . 9 6} \pm \mathbf{2 . 9 6}$ & $\mathbf{6 9 9 . 2 2} \pm \mathbf{5 3 . 0 7}$ & $\mathbf{1 5 . 6 9} \pm 3.97$ & $\mathbf{1 0 . 7 4} \pm \mathbf{0 . 9 3}$ \\
HTM & $\mathbf{7 2 . 5 3} \pm \mathbf{7 . 8 8}$ & $\mathbf{7 2 9 . 3 9} \pm \mathbf{6 9 . 4 5}$ & $\mathbf{4 9 . 3 6} \pm \mathbf{8 . 9 4}$ & $\mathbf{5 6 0 . 0 4} \pm \mathbf{6 4 . 8 2}$ & $\mathbf{1 0 . 1} \pm \mathbf{3 . 9 0}$ & $\mathbf{1 9 . 3 2} \pm 4.25$ \\
CORESTA & $\mathbf{2 1 . 5} \pm \mathbf{7 . 8}$ & $\mathbf{5 4 0 . 3} \pm \mathbf{1 3 5 . 3 0}$ & $\mathbf{4 9 . 0} \pm \mathbf{1 4 . 1 0}$ & $\mathbf{2 5 6 . 7 0 \pm 5 1 . 4 0}$ & $\mathbf{4 7 . 2} \pm \mathbf{1 4 . 6 0}$ & $\mathbf{1 3 . 2} \pm \mathbf{5 . 2 0}$ \\
\hline
\end{tabular}

${ }^{\mathrm{a} A m o u n t}$ in $\mu \mathrm{g} / \mathrm{cig}$

${ }^{\mathrm{b}}$ Each experiment was performed in triplicate, and the data are expressed as the average $( \pm \mathrm{SD})$ of the measured values.

Carbonyl compounds in exhaled breath of a smoker and non-smoker were also analyzed and the results compared. The exhaled breath of smokers was collected 20 minutes after smoking. The carbonyl profiles as determined using HTM are shown in FTICR-MS spectra (Figures 3.25). Table 3.4 provides the concentration of carbonyls 
captured by each derivatization reagent. While ATM was more effective in derivatizing formaldehyde acetone and 2-butanone than HTM, HTM was able to unmask acrolein and crotonaldehyde that could not be detected by ATM in exhaled breath of non-smokers. The amounts of unsaturated carbonyls detected by HTM were also higher than those of ATM in exhaled breath of smokers. Generally, smokers have a higher concentration of carbonyls than non-smokers except for acetone where exhaled breath of non-smoker is higher than that of smokers.

Table 3.4. The average and standard deviation of carbonyl concentrations of gaseous breath samples of a smoker and a non-smoker subject with HTM and ATM.

\begin{tabular}{lll|ll}
\hline Carbonyl VOCs & \multicolumn{2}{c|}{ HTM } & \multicolumn{2}{c}{ ATM } \\
& Non-smoker & Smoker & Non-smoker & Smoker \\
\hline formaldehyde & $0.20 \pm 0.03$ & $0.49 \pm 0.08$ & $1.03 \pm 0.44$ & $0.67 \pm 0.09$ \\
acetaldehyde & $0.16 \pm 0.013$ & $1.37 \pm 0.14$ & $0.55 \pm 0.13$ & $0.61 \pm 0.08$ \\
acetone & $4.68 \pm 1.10$ & $4.48 \pm 0.57$ & $5.75 \pm 1.03$ & $5.00 \pm 2.31$ \\
2-butanone & ND & $0.39 \pm 0.37$ & $1.81 \pm 0.08$ & $0.31 \pm 0.16$ \\
acrolein & $0.10 \pm 0.005$ & $2.02 \pm 0.20$ & ND & $0.96 \pm 0.26$ \\
crotonaldehyde & ND & $1.73 \pm 0.30$ & ND & $0.99 \pm 0.03$ \\
\hline Values in nmolL -1 & & &
\end{tabular}

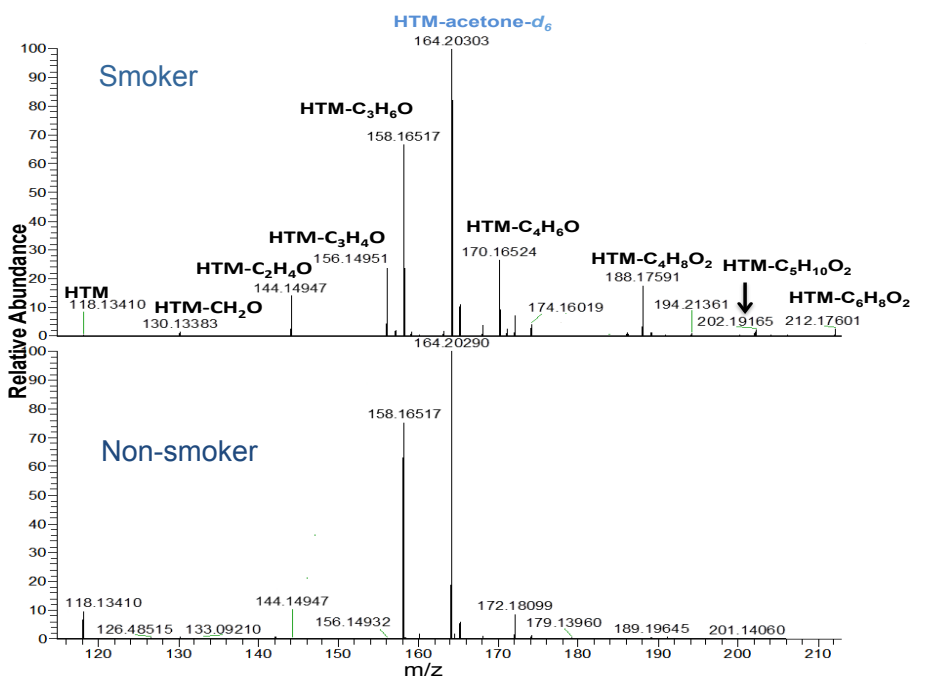

Figure 3.25. The FT-ICR-MS spectra overlay of HTM-carbonyl compounds detected in exhaled breath of a smoker and a non-smoker subjects. 


\subsection{CONCLUSION}

The kinetics of oximation reactions was studied. The reactions between ketones and aldehydes with short alkyl chain have lower activation energy, compared with carbonyl compounds with long alkyl chain. This is because the short chain carbonyl compounds have higher mixing kinetic energy than the long chain carbonyl compounds. ADMH and AMAH generally have lower activation energy than ATM although the frequency factor of ATM is generally higher than those of ADMH and AMAH. The activation energies of these reagents with acrolein and crotonaldehyde which are $\alpha, \beta-$ unsaturated aldehydes were higher than those of the saturated substrate because the $\alpha, \beta$ unsaturated are less electrophilic due to the presence of $\pi$ electrons of terminal alkene. These experimental results can be used as a guide to the choice aminooxy compounds as the coating materials for preconcentration of specific carbonyl compounds.

We have also developed a new hydrazine reagent, 2-hydrazinyl- $N, N, N$ trimethylethan-1-ammonium iodide (HTM). HTM is a hydrazine analog of ATM designed to target and chemoselectively react with $\alpha, \beta$-unsaturated carbonyls, which have hitherto been difficult to derivatize using aminooxy reagents. Both kinetic and capture efficiency studies show that derivatization of $\alpha, \beta$-unsaturated acrolein and crotonaldehyde occurs twice as efficiently with HTM than when using ATM.

The comparative analysis of carbonyl compounds in exhaled breath and tobacco cigarettes shows that the novel hydrazine reagent captures more $\alpha, \beta$-unsaturated carbonyl species than ATM. HTM could capture the low abundant acrolein and crotonaldehyde present in exhaled breath more than ATM could. 
The reactions of an aminooxy and hydrazine reagents with $\alpha, \beta$-unsaturated carbonyls are usually slow because alkene $\pi$-electrons deactivates the carbonyl carbon via resonance. However, we expected the new hydrazine reagent to be more reactive to both saturated and unsaturated carbonyls than aminooxy because of its stronger nucleophilic character. In a conjugated carbonyl system, there is an extra resonance structure that also shows electrophilic character at the terminal alkene carbon (Scheme 3.4). The resonance structures of acrolein are shown in scheme. Therefore, $\alpha, \beta-$ unsaturated aldehydes and ketones can potentially react with nucleophiles at two sites: directly at the carbonyl carbon or the end of the conjugated system (1,4-addition). In direct addition, the nucleophile attacks "directly" at the carbonyl $(\mathrm{C}=\mathrm{O})(1,2$-addition). These are two competing reactions. However, we did not detect the 1,4-addition product. The electron density that could be supplied to the carbonyl carbon by the terminal $\pi$-electrons likely lowered the 1,2-addition reaction. However, the reason for higher reactivity of aminooxy ATM towards saturated carbonyl compounds is not known and needs to be investigated.

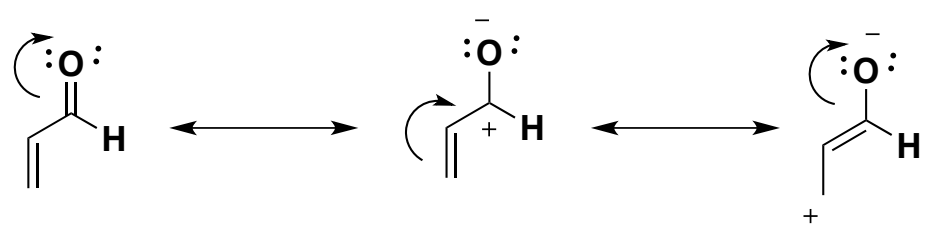

Scheme 3.4. Resonance structure of acrolein. 


\subsection{EXPERIMENTAL SECTION}

\subsubsection{Materials and methods}

All reagents and solvents, including deuterated acetone (acetone- $\left.d_{6}\right)$ (99.9\%), acetone $(99 \%)$, propanal $(99 \%), 2$-pentanone, propanal, acrolein $(\geq 99.5 \%)$, crotonaldehyde ( $\geq 99.5 \%$, mixture of cis and trans), 2-dimethylaminoethanol $(\geq 99.5 \%)$, thionyl chloride, hydrazine monohydrate (98\%), and methanol (99.9\%), were purchased from Sigma-Aldrich. Acrolein (99.5\%) was purchased from Fluka Analytical and methyl iodide was purchased from Alfa Aesar. Reagents ATM (1), ${ }^{141}$ and AMAH (2) ${ }^{55}$ (Figure 3.3.0) were prepared according to literature procedures. The Kentucky reference cigarette 3R4F was purchased from the University of Kentucky College of Agriculture Reference Cigarette Program. The silicon microreactors were fabricated from 4"-silicon wafers using standard microelectromechanical systems techniques. Details of the microreactor design and fabrication have been published elsewhere. ${ }^{31,47,54}$

\subsubsection{Measurement of reaction kinetics}

The corresponding acetone- $d_{6}$ adducts of ADMH, AMAH, ATM and HTM (5.0 $\mathrm{x} 10^{-6} \mathrm{~mol}$ ) were added (to serve as isotopically labeled internal standards) to $200 \mu \mathrm{L}$ spectroscopic grade methanol containing the respective quaternary ammonium aminooxy reagent $\left(5.0 \times 10^{-7} \mathrm{~mol}\right)$ before reaction with a carbonyl compound. Acetone $\left(5.0 \times 10^{-7}\right.$ mol), or other carbonyl compound, then was added to the reaction solution at either room temperature $\left(21^{\circ} \mathrm{C}\right), 0{ }^{\circ} \mathrm{C}$, or $-21^{\circ} \mathrm{C}$. A mixture of $90 \%$ ethylene glycol and $10 \%$ ethanol ${ }^{142}$ was used to achieve $-21^{\circ} \mathrm{C}$ and are stable for about $45 \mathrm{~min}$. Aliquots of the reaction mixture $(15 \mu \mathrm{L})$ were analyzed by FT-ICR-MS at different time intervals. The 
unreacted carbonyl compound concentration $\mathrm{C}(\mathrm{t})$ was calculated by subtraction of the reacted carbonyl compound concentration from the original concentration $\mathrm{C}_{0}$. Other kinetic studies were done following this procedure.

\subsubsection{FT-ICR-MS analysis}

The methanol solutions of the aminooxy reagent-carbonyl mixtures were analyzed on a hybrid linear ion trap FT-ICR-MS instrument (Finnigan LTQ-FT, Thermo Electron, Bremen, Germany) equipped with a TriVersa NanoMate ion source (Advion BioSciences, Ithaca, NY) with an electrospray chip (nozzle inner diameter $5.5 \mu \mathrm{m}$ ). The TriVersa NanoMate was operated in positive ion mode by applying $2.0 \mathrm{kV}$ with no head pressure. Initially, low-resolution MS scans were acquired for $1 \mathrm{~min}$ to ensure the stability of ionization, after which high mass accuracy data were collected using the FTICR analyzer where MS scans were acquired for $8.5 \mathrm{~min}$ and at the target mass resolution of 100,000 at $800 \mathrm{~m} / \mathrm{z}$. The quaternary aminooxy compound and its adducts were assigned on the basis of their accurate mass by first applying a small (typically $<0.0005$ ) linear correction based on the observed mass of the internal standard.

\subsubsection{2-hydroxy-N, N, N-trimethylethan-1-ammonium iodide}

To a solution of 2-( $N, N$-dimethylamino) ethanol (1) (2.00 g, $22.44 \mathrm{mmol})$ in $\mathrm{CH}_{2} \mathrm{CH}_{2}(60 \mathrm{~mL})$ in a pressure tube was added in one portion methyl iodide $(2.10 \mathrm{~mL}$, $33.66 \mathrm{mmol}$ ). The tube was sealed and the reaction mixture was heated at $50{ }^{\circ} \mathrm{C}$ for $12 \mathrm{~h}$. On cooling, the reaction mixture was concentrated under reduced pressure in a fume hood to afford iodide salt $2(4.67 \mathrm{~g}, 90 \%)$ as a white solid that was used directly in the next 
step; mp 272-274 ${ }^{\circ} \mathrm{C}\left(272-274{ }^{\circ} \mathrm{C}^{143}\right) ;{ }^{1} \mathrm{H}$ NMR (400 MHz, DMSO-d 6$) \delta 5.32-5.12(\mathrm{t}$, $1 \mathrm{H}), 3.82(\mathrm{~d}, \mathrm{~J}=4.8 \mathrm{~Hz}, 2 \mathrm{H}), 3.40(\mathrm{dd}, \mathrm{J}=9.9,4.7 \mathrm{~Hz}, 2 \mathrm{H}), 3.11(\mathrm{~d}, \mathrm{~J}=4.1 \mathrm{~Hz}, 9 \mathrm{H})$ ppm; ${ }^{13} \mathrm{C}$ NMR (100 MHz, DMSO-d $\left.{ }_{6}\right) \delta$ 66.87, 55.1153 .15 ppm; HRMS (m/z): cal.104.1070; obt. 104.1070.

\subsubsection{2-Chloro-N, N, N-trimethylethan-1-ammonium iodide}

Alcohol 2 (2.00 g, $8.66 \mathrm{mmol})$ was dried under vacuum at $100{ }^{\circ} \mathrm{C}$ for $1 \mathrm{~h}$ immediately prior to addition of thionyl chloride $(9.48 \mathrm{~mL}, 129.8 \mathrm{mmol})$. The mixture was heated at $80{ }^{\circ} \mathrm{C}$ for $4 \mathrm{~h}$. After cooling to room temperature, unreacted thionyl chloride was removed by distillation. The residue was dissolved in methanol ( $c a .20 \mathrm{~mL})$ followed by concentration using a rotary evaporator. This procedure was repeated two more times. The solid obtained then was dried under high vacuum to give 3 ( $2.11 \mathrm{~g}$, 98\%) as a yellow crystalline solid which was used without further purification; mp 230$231{ }^{\circ} \mathrm{C}\left(228-230{ }^{\circ} \mathrm{C}{ }^{143}\right) ;{ }^{1} \mathrm{H}$ NMR $\left(400 \mathrm{MHz}, \mathrm{DMSO}_{\mathrm{d}}\right) \delta 4.11-4.02(\mathrm{t}, \mathrm{J}=6.8 \mathrm{~Hz}$, $2 \mathrm{H}), 3.77-3.67(\mathrm{t}, \mathrm{J}=6.9 \mathrm{~Hz}, 2 \mathrm{H}), 3.12(\mathrm{~s}, 9 \mathrm{H}) ;{ }^{13} \mathrm{C}$ NMR $(100 \mathrm{MHz}$, DMSO-d 6 ) $\delta$ 64.99, 52.77, 36.33; HRMS (m/z): cal.122.0731; obt. 122.0732.

\subsubsection{2-hydrazinyl-N, N, N-trimethylethan-1-ammonium iodide (HTM)}

To a stirred solution of hydrazine monohydrate $(3.31 \mathrm{~mL}, 61.00 \mathrm{mmol})$ in THF $(35 \mathrm{~mL})$ at room temperature was added iodide $3(1.50 \mathrm{~g} \mathrm{~g}, 6.12 \mathrm{mmol})$ in 3 portions over an hour. After complete addition, the reaction mixture was stirred for 30 minutes and then refluxed for $4 \mathrm{~h}$. The solvent was removed by rotary evaporation. Excess hydrazine monohydrate was distilled off and the residue crystallized by dissolving in $5 \mathrm{~mL}$ of 
boiling ethanol and allowing it to stand overnight in refrigerator to form the crystal of desired HTM. (4) (White hygroscopic solid, 0.63g, and 43\%) mp $120-121{ }^{\circ} \mathrm{C}{ }^{1} \mathrm{H}$ NMR (400 MHz DMSO-d 6 ) $3.71(2 \mathrm{H}, \mathrm{m}), 3.16(9 \mathrm{H}, \mathrm{s}), 3.05$ (2 H, dd, J 10.2, 6.6); ${ }^{13} \mathrm{C}$ NMR (100 MHz, DMSO-d 6 ); 62.40, 52.95, 46.95; HRMS (m/z): cal.118.1339; obt. 118.1341.

\subsubsection{Exhaled breath analysis}

The exhaled breath of a smoker and a non-smoker was collected. That of a smoker was collected 20 minutes after smoking. The preconcentration and derivatization of exhaled breath samples from a smoker and a non-smoker were achieved by the microreactors coated with $0.5 \mu \mathrm{mol}$ of HTM or ATM. Exhaled breath sample was pulled through the microreactor and evacuated under vacuum at a flow rate of $3.5 \mathrm{~mL} / \mathrm{min}$. The eluted solutions were analyzed by FT-ICR-MS. 4 nmol of internal standard was added to each sample and quantification achieved using calibration curves.

\subsubsection{Cigarette smoke analysis}

The method of derivatization of the carbonyl compounds generated from cigarette smoke is similar to that of the exhaled breath. A Kentucky reference cigarette 3R4F was burnt and the smoke collected with a $100 \mathrm{~mL}$ syringe fitted with rubber tubing. $60 \mathrm{~mL}$ of smoke was collected per puff and a total of 9 puffs were collected into a 10 Liter tedlar bag. $2 \mathrm{~mL}$ aliquot was taken out and injected into a $500 \mathrm{~mL}$ tedlar bag and made up to $500 \mathrm{~mL}$ with $498 \mathrm{~mL}$ pure air with a cylinder. The Tedlar bag was connected to the microchip loaded into which $6.25 \times 10^{-7}$ mol of the HTM or ATM, evacuated, and analyzed as described on electronic cigarette aerosol evacuation with an optimal flow rate 
of $3 \mathrm{~mL} / \mathrm{min}$. After evacuation, the chip is eluted into a vial having an insert with $200 \mu \mathrm{L}$ $\mathrm{MeOH}$ followed by the addition of $4 \mathrm{nmol}$ internal standard. The spectra are obtained with FTICRMS and quantification of carbonyls achieved with calibration curves. 
CHAPTER 4 NICOTINE IN E-CIG LIQUIDS AND DERIVED AEROSOLS

4.1. INTRODUCTION

4.2. STANDARD METHOD FOR NICOTINE COLLECTION AND QUANTIFICATION

4.3. EXPERIMENTAL DESIGN FOR NICOTINE SAMPLE COLLECTION AND ANALYSIS

4.4 RESULTS AND DISCUSSION

4.5 CONCLUSION

4.6 EXPERIMENTAL SECTION 


\subsection{INTRODUCTION}

Nicotine is a toxic, potent alkaloid that is quickly absorbed through the skin and mucous membranes in its neutral form. ${ }^{144}$ Nicotine has two nitrogen atoms capable of accepting a proton. The pyrrolidine nitrogen is more basic than the pyridine nitrogen (Figure 4.0). ${ }^{145}$

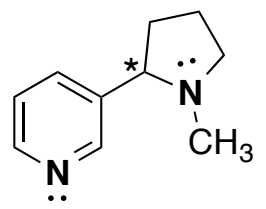

Figure 4.0. The structure of nicotine $(*=$ stereogenic center $)$.

Nicotine plays a significant role in the development of cardiovascular disease. ${ }^{146}$ Nicotine is known to constrict blood vessels and reduce the flow of blood to the hands and feet. In addition to its central nervous system effects, nicotine also inhibits the release of prostacyclin, a vasodilation prostaglandin, from vascular tissue and induces hormonal changes associated with hypothalamic pituitary axis (HPA). ${ }^{147}$ The health effects, which are of major concern, include coronary artery and peripheral vascular disease, hypertension, peptic ulcer disease, and reproductive disorders. ${ }^{148}$ Nicotine has been implicated in stimulating neuroendocrine tumor cell line proliferation, ${ }^{149}$ a factor in the pathogenesis of lung cancer ${ }^{150}$ and apoptosis prevention. ${ }^{151}$ A lethal dose of nicotine in humans is 30-60 mg. ${ }^{152}$

Nicotine levels in e-liquids are intentionally formulated to create target strengths, yet measured levels may not match the manufacturer's claim. There are a variety of e-liquids, including flavored e-liquids, with different formula and 
nicotine strength (typically from 0 to $36 \mathrm{mg} / \mathrm{mL}$ ). The efficacy of nicotine delivery by e-cigarettes is not well understood. Consequently, there is a great public health concern regarding exposure to harmful chemicals in aerosols of ecigarettes. ${ }^{88,153,117}$ Therefore, there is a need for efficient measurement of nicotine in e-liquids and in the derived aerosols of e-cigarettes as well as better evaluation of the efficacy of nicotine delivery by electronic nicotine delivery devices (ENDs).

\subsubsection{Properties of nicotine}

Nicotine is a colorless liquid, which turns to a yellow-brown oily liquid over time or on exposure to sunlight. It is hygroscopic and miscible with water in its basic form. It has a flash point of $95{ }^{\circ} \mathrm{C}$ and an autoignition temperature of $224{ }^{\circ} \mathrm{C}$ with a vapor pressure of $5.5 \mathrm{~Pa}$ at $25^{\circ} \mathrm{C}$. Nicotine is optically active and exists as two enantiomers.

The naturally occurring form of nicotine has $(S)$ configuration $^{86}$ and is levorotatory with a specific rotation of $[\alpha]_{D}=-166.4^{\circ}((-)$-nicotine). The dextrororatory form, $(+)$-nicotine, is physiologically less active than (-)-nicotine. (-)-Nicotine is more toxic than (+)nicotine. The salts of (+)-nicotine are usually dextrorotatory. The hydrochloride and sulphate salts become optically inactive if heated in a closed vessel above $180^{\circ} \mathrm{C}$. Nicotine can occurs naturally in the leaves of Nicotina rustica and tobacco plants. ${ }^{154}$

\subsubsection{Nicotine biosynthesis}

Nicotine is synthesized by condensation of an intermediate in the nicotinamide adenine dinucleotide (NAD) salvage pathway and the methylpyrrolinium cation derived from ornithine via putrescine. This cation is also used for biosynthesis of tropane 
alkaloids, such as hyoscyamine and scopolamine. Enzymes involved in nicotine synthesis include: ODC; ornithine decarboxylase, PMT; putrescine Nmethyltransferase, DAO; diamine oxidase, AO; aspartate oxidase, QS; quinolinate synthase, and QPT; quinolinate phospho-ribosyltransferase (Scheme 4.0).

The nicotine pyrrolidine ring is derived from $N$-methylpyrrolinum cation, which is a spontaneous cyclization product of the oxidative deamination reaction from $\mathrm{N}$ methylputrescine catalyzed by diamine oxidase (DAO). $N$-Methylputrescine is produced from putrescine by putrescine $N$-methyltransferase (PMT) ${ }^{155}$ The nicotine pyridine ring is formed from the NAD biosynthetic pathway, ${ }^{156-157}$ however it is not clear whether nicotinic acid itself or a metabolite derivative is the direct precursor of nicotine. The amino acid sequence of PMT is highly homologous to the sequence of spermidine synthase (SPDS), which transfers the amino-propyl moiety of decarboxylated $S$ adenosylmethionine (dSAM) to putrescine. PMT catalyzes a transfer of the methyl moiety of $S$-adenosylmethionine (SAM) to putrescine. It is assumed that PMT evolved from SPDS after restricted alterations of critical dSAM binding amino acid residues. Tobacco DAO may have been evolved from a DAO widespread in nature by optimization of substrate specificity. The DAOs involved in nicotine and tropane alkaloid biosynthesis have higher affinity for $\mathrm{N}$-methylputrescine than for putresine and other symmetrical diamines. ${ }^{158}$ 


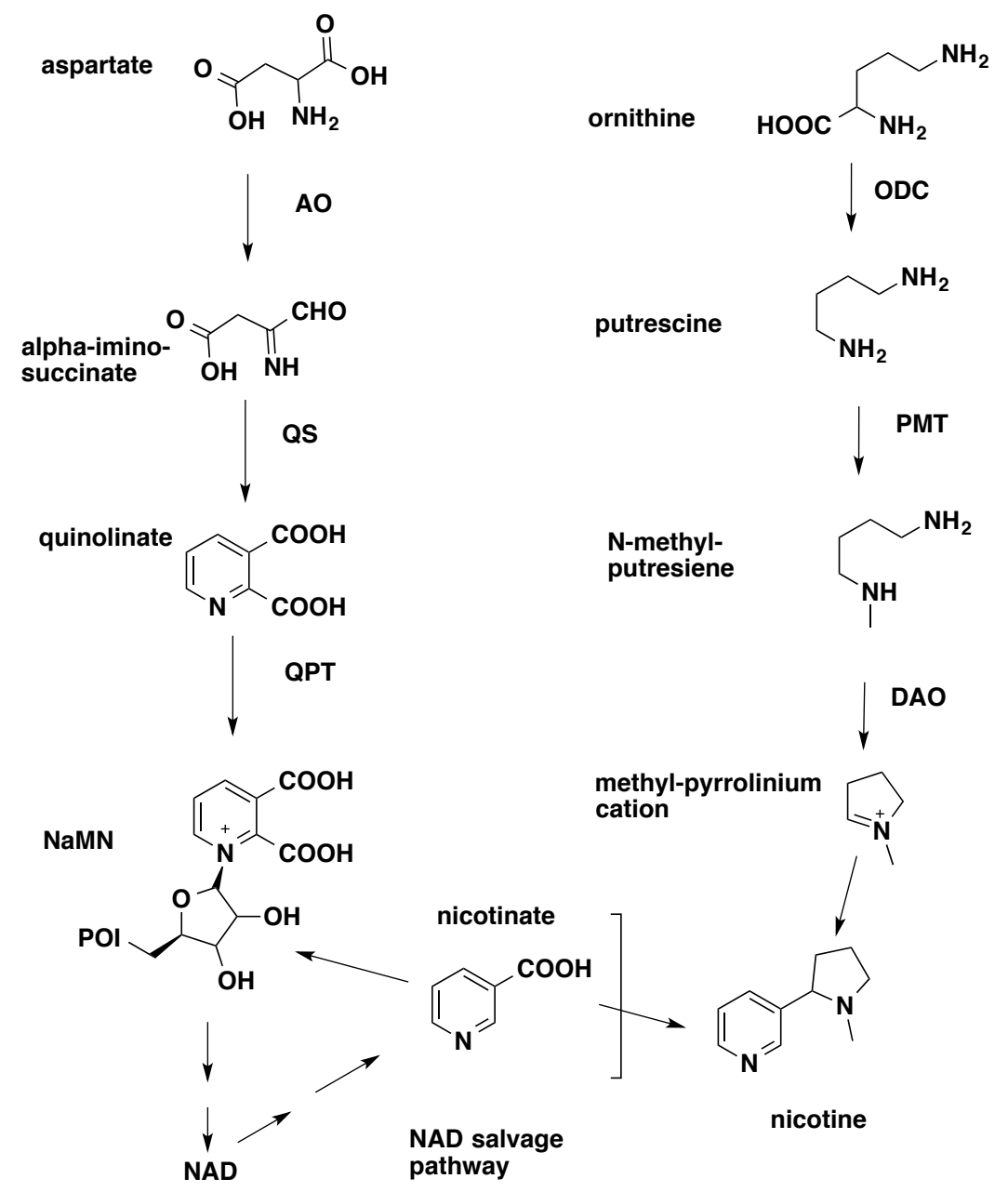

Scheme 4.0. Biosynthetic pathway of nicotine. Nicotine is synthesized by condensation of an intermediate in the NAD salvage pathway and the methylpyrrolinium cation derived from ornithine via putrescine.

\subsection{STANDARD METHOD FOR NICOTINE COLLECTION AND QUANTIFICATION}

The standard method for collection and analysis of nicotine in air (National Institute for Occupational Safety and Health, NIOSH 2551) requires a packed sorbent tube, usually XAD-4 sorbent (Figure 4.1), to trap nicotine by flowing air samples through the tube and then using ethyl acetate to desorb nicotine from the sorbent for analysis by gas chromatography (GC) or HPLC. XAD-4 sorbent is a 
styrene divinylbenzene polymer having a pore diameter of 50 Angstroms. It is suitable for low molecular weight compounds including nicotine. The method has some issues, such as nicotine partially escaping from the sorbent tube during sample collection process and inefficient desorption of nicotine from the sorbent. The process is also time-consuming and cumbersome. The analysis of nicotine in e-liquids by GC requires extraction of nicotine from the e-liquids using solvents such as ethyl acetate and toluene. ${ }^{23,82,159}$ Incomplete extraction will cause significant measurement errors. Recently, other methods were reported for collection of nicotine in the aerosols of e-cigarettes including flowing aerosols through filters or cold solvents. ${ }^{82,160}$ We therefore sought a new method for rapid analysis and quantification of nicotine in electronic cigarettes and electronic cigarette aerosols by converting nicotine to nicotinium salt (Figure 1.5) followed by rapid analysis with direct infusion FT-ICR-MS.

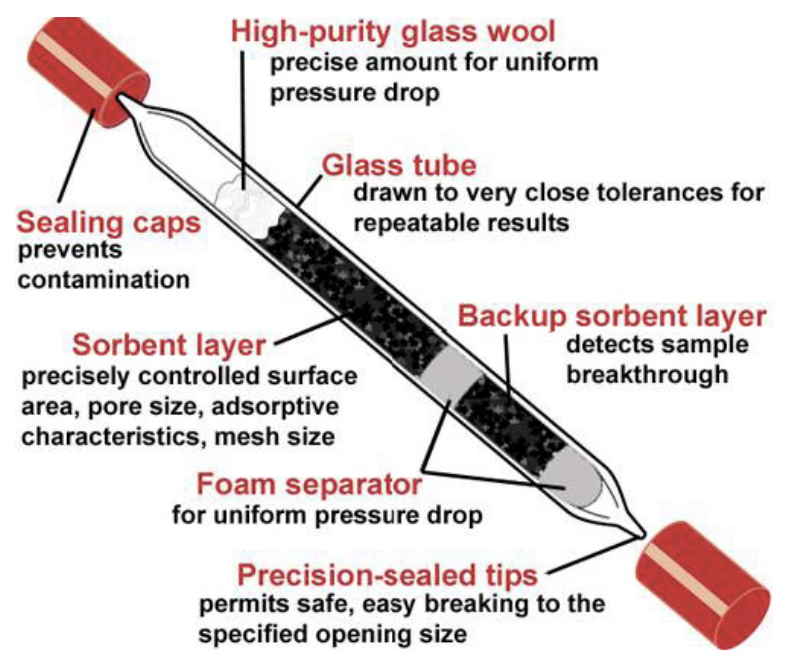

Figure 4.1. XAD-4 sorbent tube for nicotine sampling. 


\subsection{EXPERIMENTAL DESIGN FOR NICOTINE SAMPLE COLLECTION AND ANALYSIS}

Equipped with a powerful analytical tool, FT-ICR-MS, and our knowledge of nicotine $\mathrm{pkb}$, we reasoned that by protonating the pyrrolidine nitrogen with an aqueous inorganic acid, such as hydrochloric acid, to form the quaternary ammonium salt nicotinium chloride (Scheme 4.1), we could quantify the salt in ecig liquids and aerosols. This approach would circumvent most of the problems associated with the conventional standard nicotine method of analysis. Protonated nicotine in both e-liquids and as collected from derived aerosols facilitates effective and quantitative analysis by FT-ICR-MS.

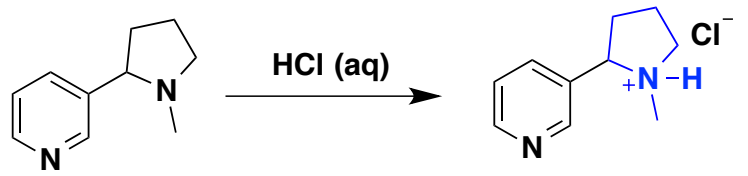

Nicotinium Chloride

Scheme 4.1. Regioselective protonation of nicotine to form the nicotinium salt.

The standard nicotine sample collection method is usually suitable for nicotine samples existing in trace levels but is not suitable for aerosol sample collection. Most sorbent tubes have limited capacity (300 $\mu \mathrm{g}$ for XAD-4) and, if exceeded, may result in breakthrough. To increase the sample collection capacity in electronic cigarette aerosol generation where several puffs will generate several micrograms to milligrams of nicotine, depending on the nicotine concentration in the e-liquid formulation, we conceived the idea of having a cold trap (an impinger) containing acidified methanol into which the aerosol would be continually delivered from a smoking robot (Figure 4.2). At the end of the puffing regime, an aliquot of the solution could then be analysed for nicotine quantification. Impinger optimization was done by connecting five impingers in 
series to determine the number of impingers that would be required for efficient aerosol collection with no nicotine loss. With electronic cigarette $\mathrm{ECC} 02$, having nicotine concentration of $15.56 \mathrm{mg} / \mathrm{mL}, 10$ puffs (puff number lower limit) and 40 puffs (puff number upper limit) were used for the study. They were delivered separately into 5 impingers connected in series and an aliquot sample from each impinger analysed for nicotine. The capture efficiency of each impinger was calculated. The first impinger trapped $74 \%$ and $78 \%$ of the total nicotine delivered with 10 and 40 puffs respectively. The second impinger trapped between 19\% and 23\% while third impingers trapped 3\% and 5\% respectively (figure 4.3). No nicotine was delivered into impingers 4 and 5 even when the nicotine concentration in e-liquid was higher. We therefore proceeded with the use of three impingers connected in series for this study.

To test this concept for nicotine analysis, we first studied the rate of protonation of the pyrrolidine ring, since we were interested in cutting down the analysis time. In order to do this we set to measure the activation energy $E_{a}$ in $\mathrm{KJ} / \mathrm{mol}$ of nicotine protonation at the pyrrolidine site.

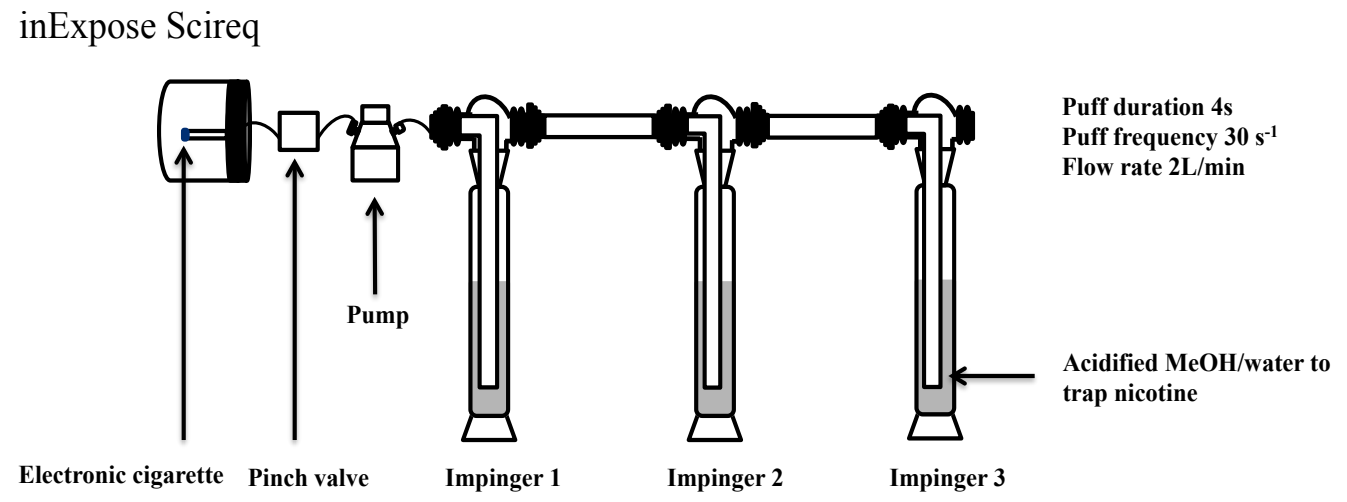

Figure 4.2. The schematic diagram of the inExpose Scireq smoking robot connected to three impingers connected in series for trapping nicotine in aerosol of e-cigarettes. 


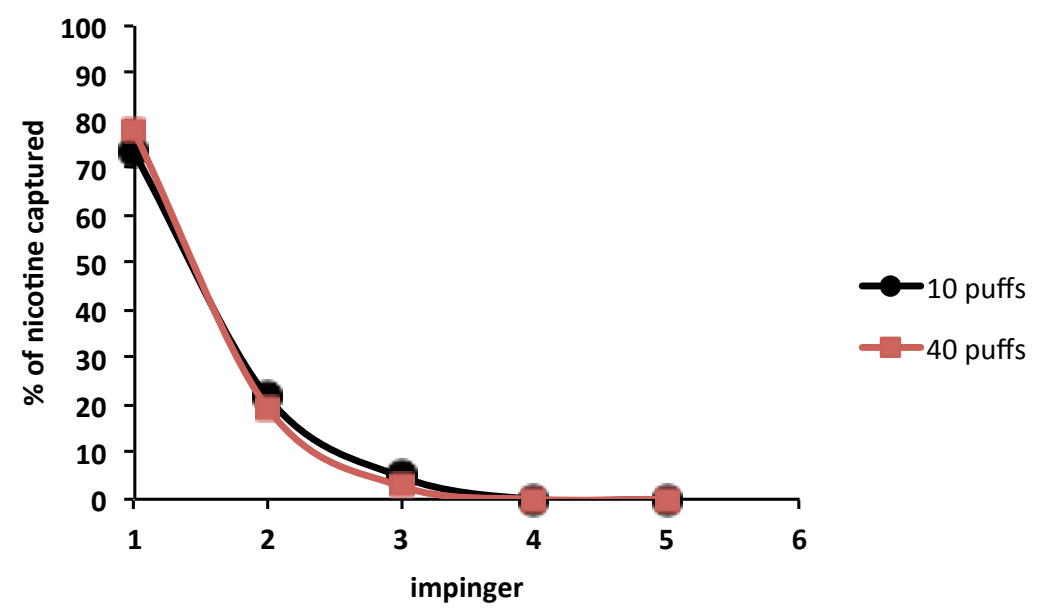

Figure 4.3. Impinger optimization: capture efficiencies of 5 impingers connected in series.

\subsection{RESULTS AND DISCUSSION}

\subsubsection{Measurement of nicotine kinetics of protonation by NMR spectroscopy}

Based on $\mathrm{p} K_{\mathrm{b}}$ considerations, El Hellani et al ${ }^{161}$ reported that nicotine is predominantly present in free base form in both e-liquids and aerosols of electronic cigarettes. Nicotine in e-liquids was extracted using toluene and amenable to analysis by GC-MS. The hypothesis of this thesis work is that protonation of nicotine in e-liquids using strong acid, such as hydrochloric acid, will form a nicotinium ion (Scheme 5.3) that can be readily measured by direct infusion FT-ICR-MS. We were gratified to learn that this approach is effective and the nicotinium cation was readily quantified using FT-ICRMS. 
To determine the rate of protonation of the pyrrolidine nitrogen of the nicotine molecule, we used nuclear magnetic resonance spectroscopy. For more efficient extraction of free nicotine for NMR analysis, we chose ethyl acetate rather than toluene. To prepare a calibration curve for measurement of nicotine using ${ }^{1} \mathrm{H}$ NMR spectroscopy, six solutions containing different amounts of nicotine dissolved in $400 \mu \mathrm{L}$ DMSO- $d_{6}$ were prepared. The concentrations of nicotine in these standards ranged from 0.78 , to 39 $\mu \mathrm{mol} / \mathrm{mL}$. A known amount of benzene was added to each standard solution as an internal reference. The integration of the benzene hydrogen signal at $\delta 7.37 \mathrm{ppm}$ was set at a constant 6 , while the integrations of the nicotine protons between $\delta 8.45-8.49 \mathrm{ppm}$ were recorded. A linear calibration curve (Fig. 4.4) shows a good linear dependence of $\mathrm{n}($ nicotine $) / \mathrm{n}$ (benzene) on $\mathrm{H}($ nicotine $) / \mathrm{H}$ (benzene), where $\mathrm{n}=$ moles and $\mathrm{H}=$ corresponding proton integration of NMR spectra. We used this plot as an NMR calibration curve to measure nicotine concentration in our kinetics study.

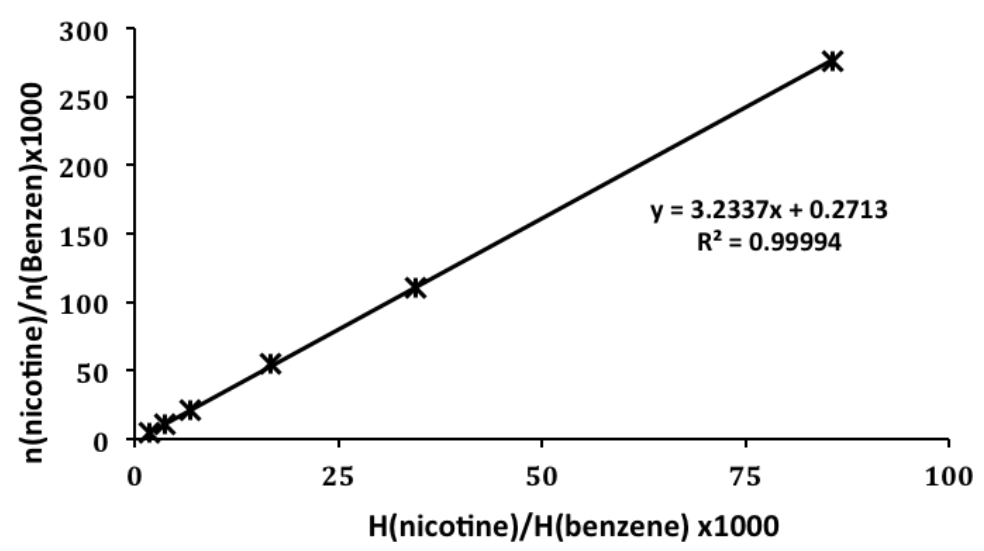

Figure 4.4. The plot of $\mathrm{n}($ nicotine $) / \mathrm{n}$ (benzene) vs. $\mathrm{H}$ (nicotine) $/ \mathrm{H}$ (benzene), serving as a calibration curve for ${ }^{1} \mathrm{H}$ NMR measurement of nicotine protonation. 
The ${ }^{1} \mathrm{H}$ NMR spectra of $5 \mu \mathrm{L}$ pure nicotine added to $400 \mu \mathrm{L}$ DMSO- $d_{6}$ was compared to the spectra from the ethyl acetate extract in $400 \mu \mathrm{L}$ DMSO- $d_{6}$ obtained after reaction of 5 $\mu \mathrm{L}$ nicotine with $\mathrm{HCl}$ in water for $30 \mathrm{~min}$ (Figure 4.5). More than $98 \%$ of nicotine was protonated and extracted into the aqueous phase based on the NMR data of this experiment.
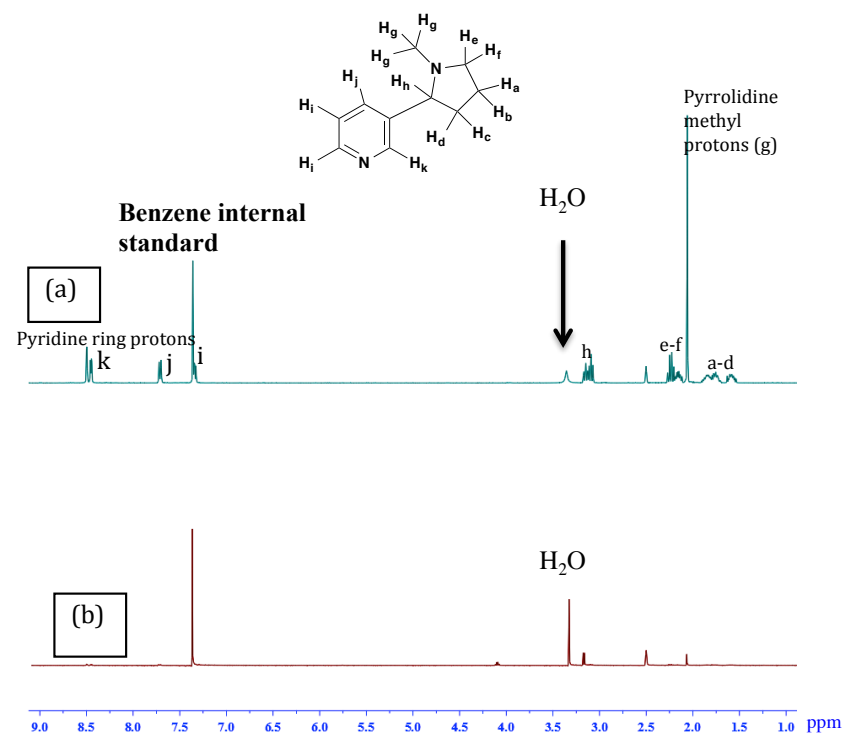

Figure 4.5. (a) ${ }^{1} \mathrm{H}$ NMR spectra (DMSO- $d_{6}$ ) of neutral nicotine and (b) ${ }^{1} \mathrm{H}$ NMR spectra $\left(\mathrm{DMSO}-d_{6}\right)$ of extracted sample after $30 \mathrm{~min}$ of protonation in water and $\mathrm{HCl}$ mixture (less than $2 \%$ of free base remains in the sample).

A kinetics study on the protonation of nicotine (Scheme 5.3) was conducted using ${ }^{1} \mathrm{H}$ NMR by measuring the amount of nicotine at different reaction times and reaction temperatures of $0,22,40$, and $60{ }^{\circ} \mathrm{C}$. Unprotonated nicotine was extracted with ethyl acetate from acidified (protonated) nicotine solution in water at a given temperature. With the addition of benzene as an internal standard, the amount of basic nicotine that was not protonated was measured. Complete conversion of nicotine to nicotinium was achieved at $22{ }^{\circ} \mathrm{C}$ in 60 minutes (Figure 4.6). 


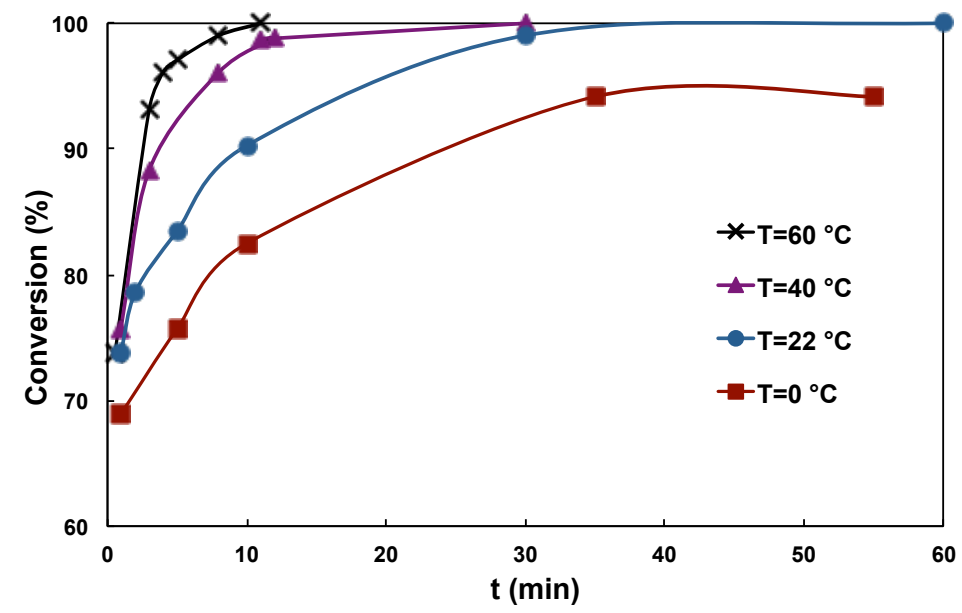

Figure 4.6. Percent conversion of nicotine to its nicotinium salt at different temperatures over time.

The first order reaction kinetics for nicotine protonation under the experimental conditions can be written as in equation 1, where [nicotine] indicates the concentration of nicotine at $t$ minutes and $\mathrm{k}$ is the apparent first order reaction rate coefficient.

$$
\mathrm{d}[\text { nicotine }] / \mathrm{dt}=-\mathrm{k}[\text { nicotine }]
$$

Integration of both sides of equation 1 and rearrangement affords equation 2 ,

$$
\ln [\text { nicotine }]=-k t+\ln [\text { nicotine }]_{\mathrm{o}}
$$

where $[\text { nicotine }]_{0}$ denotes the initial concentration of nicotine. Taking the natural logarithm of Arrhenius' equation yields equation 3,

$$
\ln k=-E_{\mathrm{a}} / R T+\ln A
$$

where $E_{\mathrm{a}}$ is the activation energy, $R$ is the universal gas constant, $T$ is the reaction temperature, and $A$ is the pre-exponential factor. 
The plotted results of $\ln \left[\right.$ nicotine] vs. time $t$ at $0,22,40$, and $60{ }^{\circ} \mathrm{C}$ demonstrated a good linear relationship in agreement with equation 2, and validated the first order reaction kinetics (Figure 4.7). Figure 4.8 shows the slope of the plot of $\ln k$ vs. the reciprocal of $\mathrm{T}$ and the intercept $(\ln A)$. When applying equation 3, $A$ is obtained as 2.46 $\mathrm{x} 10^{5} \mathrm{~min}^{-1}$ and the activation energy $\left(E_{\mathrm{a}}\right)$ for nicotine protonation is $30.05 \mathrm{~kJ} \mathrm{~mol}^{-1}$.

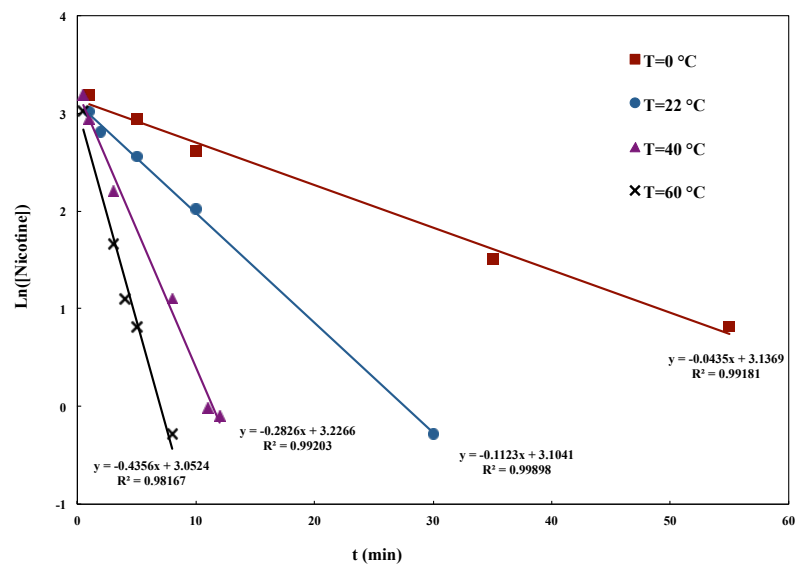

Figure 4.7. Dependence of $\ln \left[\right.$ nicotine] on the reaction temperature $\mathrm{T}\left({ }^{\circ} \mathrm{C}\right)$.

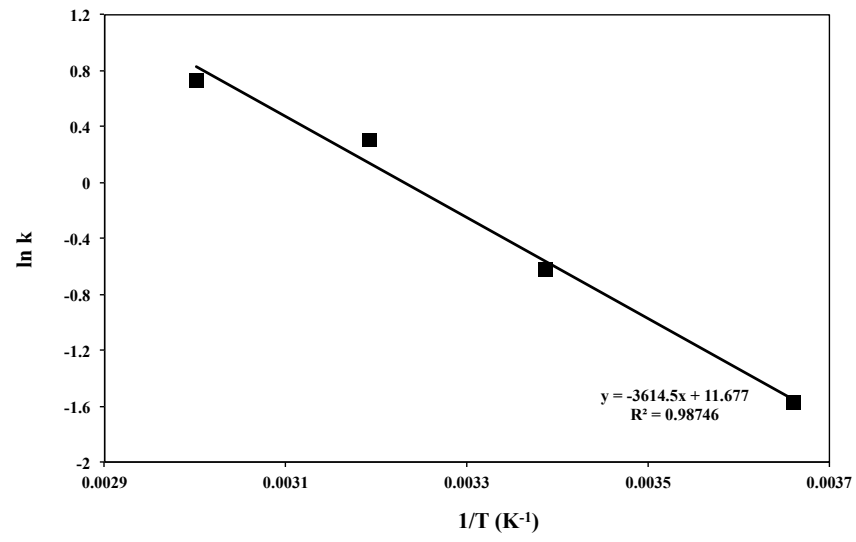

Figure 4.8. The relationship between $\ln \mathrm{k}$ and $1 / \mathrm{T}$ for protonation of nicotine in $\mathrm{HCl}$ solution. 


\subsubsection{Calibration curve of protonated nicotine by FT-ICR-MS}

Having studied rates of nicotine protonation, we proceeded to measure nicotine in e-liquids and aerosols of electronic cigarettes using FT-ICR-MS. A nicotine calibration curve using FT-ICR-MS for quantitative measurements was obtained by plotting the ratio of the relative abundance of nicotine to an internal standard (y) against the mole ratio of the analyte to the internal standard (x). Tetraethylammonium bromide was initially selected as the internal standard because of its stability and lack of volatility, but our inability to obtain reproducible results led us to select deuterated nicotinium (nicotinium$d_{3}$ ) chloride as the internal standard, which gave reproducible measurements. In addition, the deuterated nicotinium ion, [nicotine- $\left.d_{3}-\mathrm{H}\right]^{+}$, gave a strong and stable signal ion in FTICR-MS at $m / z=164.1418$. In this study, a fixed amount of $7.78 \mathrm{nmol}$ of protonateddeuterated nicotine was added to serially diluted protonated nicotine solutions as an internal reference to obtain a calibration curve. Figure 5.10 shows the FT-ICR-MS spectra overlay of the calibration samples at different protonated nicotine concentrations. Figure 4.9 shows the calibration curve of nicotine measured by FT-ICR-MS that was used to determine the amount of nicotine in all e-liquids as well as the collected aerosols for puff-by-puff nicotine delivery measurements. The calibration curve showed linearity between the intensity ratio of nicotine-to-nicotine- $d_{3}$ (I-Nic/I-Nic- $\left.d_{3}\right)$ and the molar ratio of nicotine-to-nicotine- $d_{3}\left(\mathrm{M}-\mathrm{Nic} / \mathrm{M}-\mathrm{Nic}-d_{3}\right)$ in the working mole ratio range of nicotineto-nicotine-d3 from 1 to $13\left(\mathrm{y}=1.1323 \mathrm{x}-0.3315, \mathrm{R}^{2}=0.99388\right)$. The insert in Figure 4.9 shows the linearity between the intensity ratio of nicotine-to-nicotine- $d_{3}$ and the molar ratio of nicotine-to-nicotine- $d_{3}$. Figure 4.10 shows the FT-ICR-MS of the spectra of standard calibration curve working solutions, each spiked with $7.78 \mathrm{nmol}$ nicotinium- $d_{3}$ 
as an internal standard. The nicotine peak grows relative to the standard peak as the nicotine concentration increases. The calibration curve was used to quantify nicotine in eliquid and their derived aerosols. To determine the limit of detection (LOD) for nicotine, a series of low concentration nicotine samples were prepared and analyzed by FT-ICRMS. The LOD of nicotine defined as nicotine signal-to-noise ratio of $3(\mathrm{~S} / \mathrm{N}=3)$ was obtained as $1 \times 10^{-12} \mathrm{~mol} / \mathrm{L}$.

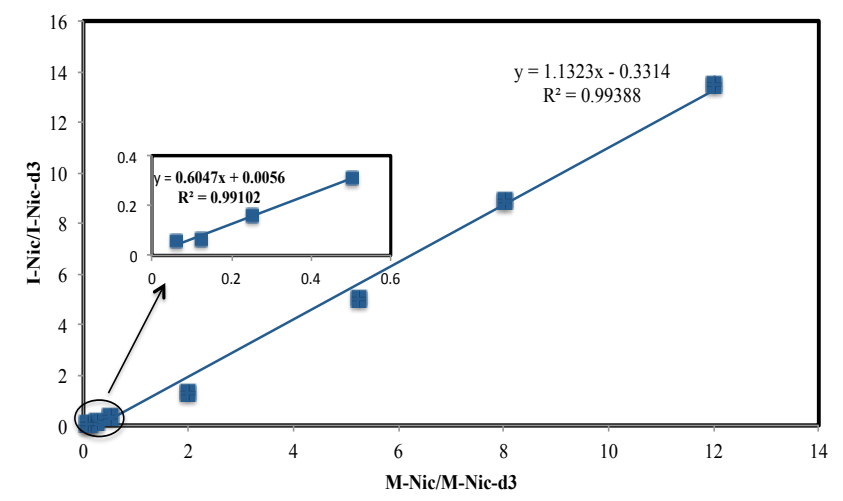

Figure 4.9. The calibration curve of nicotine by plotting the ratio of intensity of nicotineto-nicotine- $d_{3}$ (I-Nic/I-Nic- $\left.d_{3}\right)$ against the ratio of the amounts (mole) of nicotine-tonicotine- $d_{3}\left(\mathrm{M}-\mathrm{Nic} / \mathrm{M}-\mathrm{Nic}-d_{3}\right)$.

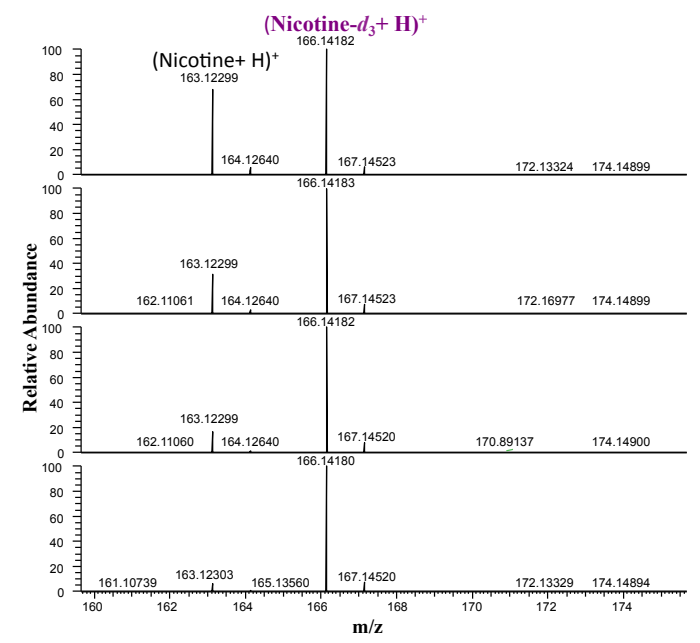

Figure 4.10. Comparison of FT-ICR-MS spectra of standard calibration curve working solutions, each spiked with 7.78 nmol nicotinium- $d_{3}$ as an internal standard. The nicotinium peak increases relative to the standard peak as the nicotinium concentration increases. 


\subsubsection{Calibration curve of nicotine by GC-MS}

To compare our novel method of nicotine analysis with standard method for collection and analysis of nicotine in air (National Institute for Occupational Safety and Health, NIOSH 2551) using GC-MS, a calibration curve was required. Quinoline was as used internal standard and Method 2551 was modified and for nicotine analysis. ${ }^{162} \cdot 9.9 \mu \mathrm{L}$ of nicotine was diluted to $10 \mathrm{~mL}$ ethyl acetate solution of triethylamine (desorbing solvent) to give $100 \mathrm{mg} / \mathrm{mL}$. A modified ethyl acetate solution is ethyl acetate containing $0.01 \%$ triethylamine. $100 \mu \mathrm{L}$ of the nicotine solution was mage up to $10 \mathrm{~mL}$ with the desorbing solution. A serial dilution of the solution was made with a working range of containing $3.9 \mathrm{nmol}$ to $0.12 \mu \mathrm{mol}$ of nicotine. A nicotine calibration curve using GC-MS for quantitative measurement was obtained by plotting the mole ratio of nicotine to an internal standard (y) against the peak area of the analyte to the internal standard (x). Figure 4.11 shows the calibration curve of nicotine measured by GC-MS that was used to determine the amount of nicotine in some e-liquids as well as the collected aerosols for puff-by-puff nicotine delivery measurements. The calibration curve showed an excellent linearity between the mole ratio of nicotine-to-quinoline (Amt of Nicotine/Amt IR) and peak area ratio of nicotine-to-quinoline (Area of nicotine/Area of IR) $\left(y=0.5641 x+0.2083, R^{2}=0.99192\right)$. Figure 4.12 shows the GC chromatogram overlay of selected calibration working samples at different nicotine concentrations. 


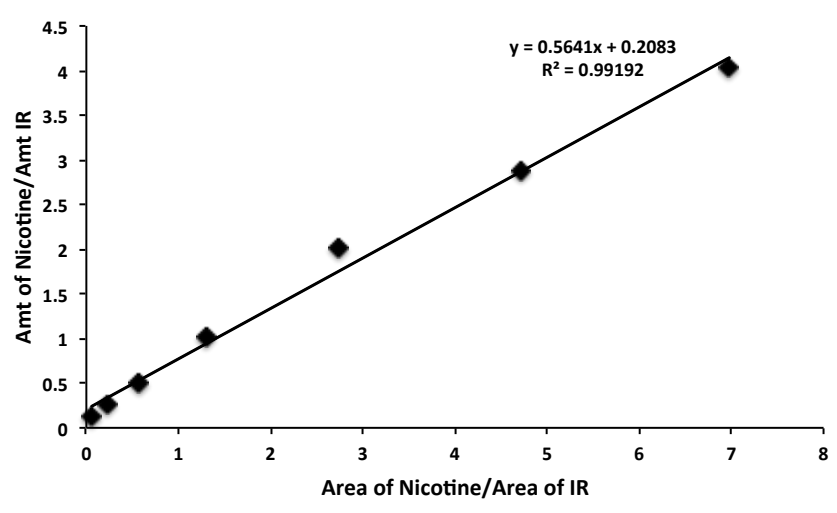

Figure 4.11. The calibration curve of nicotine built by plotting ratio of mole of nicotineto-quinoline against the ratio of peak area of nicotine-to-quinoline.

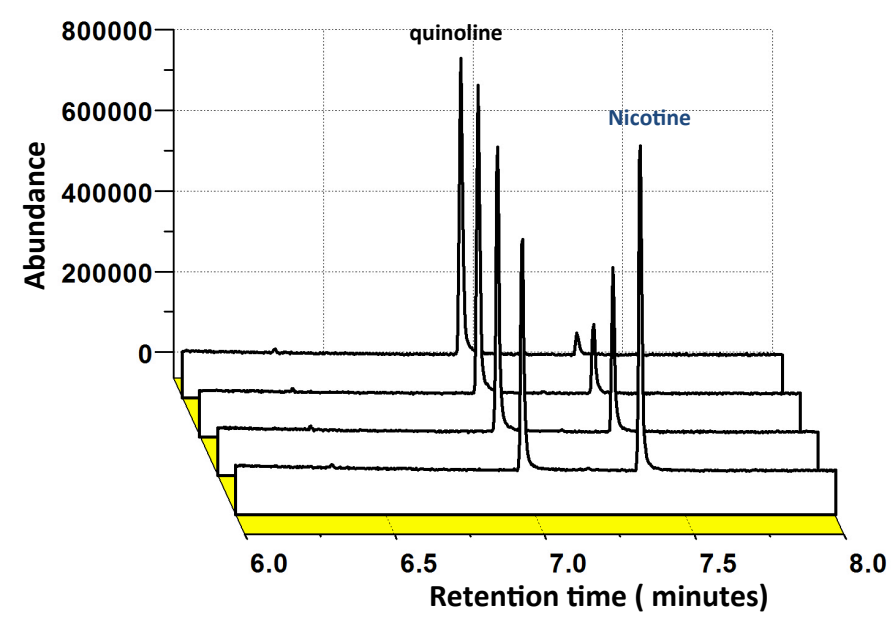

Figure 4.12. Comparison of GC chromatogram of standard calibration curve working solutions, each spiked with $40 \mathrm{nmol}$ quinoline as an internal standard. The nicotine peak grows relative to the standard peak as the nicotine concentration increases.

\subsubsection{Nicotine levels in e-liquids}

To validate the method of protonation of nicotine in e-liquids and analysis by FT-ICR-MS, a known amount of nicotine was spiked into a PG/VG (50/50) mixture (zero nicotine e-liquid). One aliquot of the mixture was used for protonation of nicotine described in e-liquids and analyzed by FT-ICR-MS. The other aliquot was used for extraction of nicotine from the PG/VG mixture to ethyl 
acetate and analyzed by GC-MS. The results indicated that the FT-ICR-MS analysis was within $2 \%$ difference from the spiked amount of nicotine, while the GC-MS analysis could be 10 to $15 \%$ less than the spiked amount of nicotine due to the inefficiency of ethyl acetate extraction. After validation of the method of protonation of nicotine in e-liquids, popular brands of e-liquids (shown in Table 5.4) were examined for nicotine content. The measured nicotine values for all eliquid brands in Table 5.4 had standard deviations less than 7\%. Of the 10 brands tested, we found that the range difference between the measured nicotine content and the manufacturer-specified content ranged from $-2.9 \%$ to $25.2 \%$.

Table 4.0. Results of nicotine analysis from selected commercial e-liquids. Each sample was analyzed in triplicate and the data are expressed as the average $[ \pm \mathrm{SD}]$ of the measured values.

\begin{tabular}{|c|c|c|c|c|c|c|c|}
\hline $\begin{array}{l}\text { EC } \\
\text { code }\end{array}$ & $\begin{array}{l}\text { Brand } \\
\text { name }\end{array}$ & Model/Flavor & Country & $\begin{array}{l}\text { Source } \\
\text { of } \\
\text { product }\end{array}$ & $\begin{array}{l}\text { Vendor's } \\
\text { Claim } \\
\text { nicotine } \\
(\mathrm{mg} / \mathrm{mL})\end{array}$ & $\begin{array}{l}\text { Nicotine } \\
(\mathrm{mg} / \mathrm{mL}) \\
\text { measured }\end{array}$ & $\begin{array}{l}\% \\
\text { Difference }\end{array}$ \\
\hline EL01 & EVO & Blackdiamond & USA & Kiosk & 6.00 & $6.28 \pm 0.17$ & 4.67 \\
\hline EL02 & NicQuid & Smooththol & USA & Kiosk & 6.00 & $6.54 \pm 0.41$ & 9.00 \\
\hline EL03 & $\begin{array}{l}\text { Perfected } \\
\text { Vapes }\end{array}$ & Clearwater & USA & Kiosk & 6.00 & $7.04 \pm 0.40$ & 17.33 \\
\hline EL04 & Halo & Menthol Ice & USA & Online & 6.00 & $6.53 \pm 0.20$ & 8.83 \\
\hline EL 05 & Halo & Mocha Café & USA & Online & 6.00 & $6.42 \pm 0.05$ & 7.00 \\
\hline EL06 & Halo & Southern Cafe & USA & Online & 6.00 & $6.26 \pm 0.05$ & 4.33 \\
\hline EL07 & NJOY & Classic Tobacco & USA & Online & 10.00 & $12.25 \pm 0.53$ & 25.20 \\
\hline EL08 & NJOY & Classic Tobacco & USA & Online & 15.00 & $16.22 \pm 0.72$ & 8.13 \\
\hline EL09 & VaporFi & Classic Tobacco & USA & Online & 18.00 & $17.47 \pm 0.45$ & -2.94 \\
\hline EL10 & VaporFi & Classic Tobacco & USA & Online & 36.00 & $37.22 \pm 1.26$ & 3.39 \\
\hline ECC01 & Blu & $\begin{array}{l}\text { Magnificent } \\
\text { Menthol }\end{array}$ & USA & Online & $13-16$ & $14.14 \pm 0.73$ & $\begin{array}{l}8.76 \text { to } \\
11.63\end{array}$ \\
\hline $\mathrm{ECC} 02$ & Blu & Classic Tobacco & USA & Online & $13-16$ & $15.67 \pm 1.01$ & $\begin{array}{l}20.53 \text { to } \\
2.06)\end{array}$ \\
\hline
\end{tabular}

\subsubsection{Nicotine levels in e-cigarette aerosols}

The smoking robot was used to generate aerosols from blu plus ${ }^{\circledR}$ e-cigarette filled with a number of e-liquids. The generated aerosol flowed through three 
traps (impingers) connected in series. Each experiment was performed in triplicate and the data were reported as the average $[ \pm \mathrm{SD}]$ of the measured values. Figure 4.13 shows the plots of nicotine in aerosol vs. the puff number for five different eliquids with measured actual nicotine level from $6.28 \mathrm{mg} / \mathrm{mL}$ to $37.22 \mathrm{mg} / \mathrm{L}$ shown in Table 5.4. There is a good linear relationship between the amount of nicotine in the aerosols and the puff number in the studied puff number range. The measurements indicate the difference of nicotine in aerosols even for 10 puffs for the blu plus ${ }^{\circledR}$ magnificent methanol (measured nicotine $14.14 \mathrm{mg} / \mathrm{mL}$ ) and classic tobacco cartridges (measured nicotine $15.67 \mathrm{mg} / \mathrm{mL}$ ) even though both cartridges were labeled as $13-16 \mathrm{mg} / \mathrm{mL}$ of nicotine level. Table 5.5 shows a comparison of measured nicotine in aerosols of e-cigarettes collected by this impinger method and the NIOSH 2551 sorbent method. The results indicate that nicotine collected by the sorbent tubes were consistently lower than that collected by the impinger method (Table 5.5). Figure 4.14 shows plots of measured nicotine concentration in the e-liquids vs. the amount of nicotine in aerosols at constant puff numbers of 20, 30, and 40. Again, there is a good linear relationship between measured nicotine concentrations in these e-liquids vs. measured nicotine amount in aerosols at the same total puff numbers. These results show that the amount of nicotine in aerosols depends on both its level in e-liquids and number of puffs.

The average nicotine levels in aerosols for a single puff at the puff volume of $91 \mathrm{~mL} /$ puff were from $18 \mu \mathrm{g}$ (eVo e-liquid, $6.28 \mathrm{mg} / \mathrm{mL}$ nicotine) $72 \mu \mathrm{g}$ (blu plus ${ }^{\circledR}$ classic, $15.67 \mathrm{mg} / \mathrm{mL}$ nicotine). Previous publications indicate nicotine levels from a single puff volume of $70 \mathrm{~mL}$ in e-liquids was between 1.7 and 51.3 
$\mu \mathrm{g} .{ }^{17}$ However, both the nicotine levels in e-liquids and cartridges and the puff volume are different. Therefore, it is hard to make a meaningful comparison. A dose inhaled from one conventional cigarette smoke was measured from 1.54 to $2.0 \mathrm{mg} .{ }^{23}$ If assuming a series of 10 puffs of e-cigarettes is equivalent to smoking one tobacco cigarette, the e-liquids might deliver $0.18 \mathrm{mg}$ to $0.72 \mathrm{mg}$ nicotine, which is much lower than one tobacco cigarette. Our results confirm previously reported findings. ${ }^{163}$ However, if the e-liquid with the highest nicotine level of $37.22 \mathrm{mg} / \mathrm{mL}$ is used, the e-cigarette may deliver $2.3 \mathrm{mg}$ for 10 puffs of aerosols, which is comparable with nicotine inhaled from the mainstream smoke of one tobacco cigarette. A survey of e-liquid market indicates that some e-liquid manufactures provide the highest strength of 36 to $42 \mathrm{mg} / \mathrm{mL}$ of nicotine in eliquids.

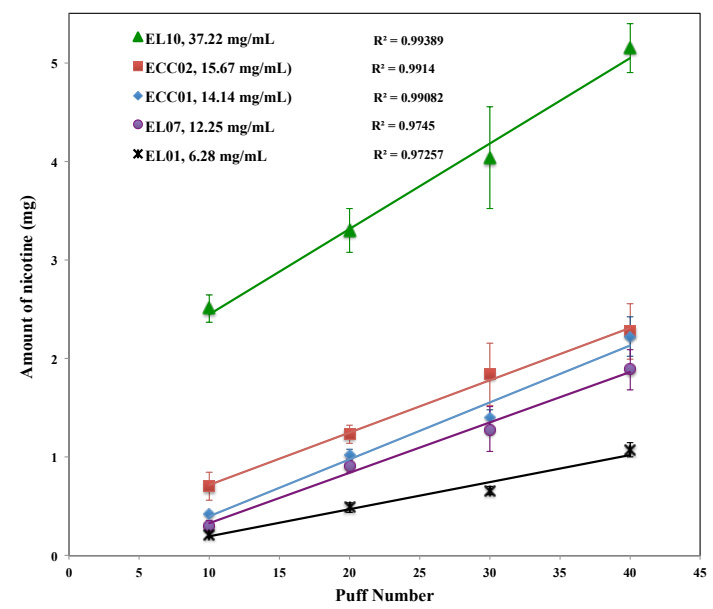

Figure 4.13. Nicotine delivery profile of e-cigarette cartridges and e-liquids with different nicotine levels. 


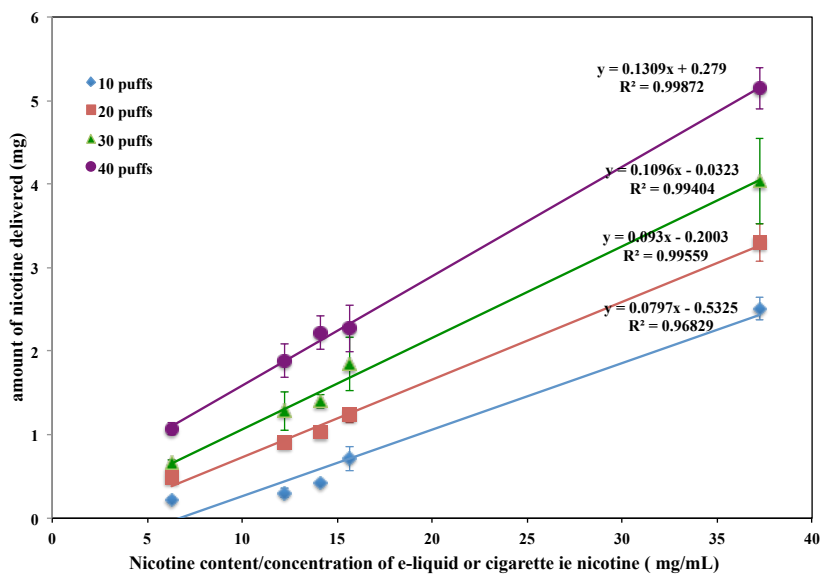

Figure 4.14. The relationship between nicotine aerosol and nicotine levels in e-liquids at constant puff numbers.

Table 4.1. Comparison of measurements of nicotine in aerosol samples collected by sorbent tube and impinger methods.

\begin{tabular}{|l|l|c|c|c|}
\hline \multicolumn{2}{|c|}{ E-liquids } & EL01 $(\mathrm{mg})$ & ECC01 $(\mathrm{mg})$ & EL10 (mg) \\
\hline \multirow{2}{*}{10 puffs } & Sorbent & 0.104 & 0.184 & 2.07 \\
\cline { 2 - 5 } & Impinger & 0.214 & 0.422 & 2.51 \\
\hline \multirow{2}{*}{20 puffs } & Sorbent & 0.214 & 0.406 & 2.86 \\
\cline { 2 - 5 } & Impinger & 0.488 & 1.03 & 3.29 \\
\hline
\end{tabular}

\subsection{CONCLUSION}

We have developed a new method for collection and analysis of nicotine in the electronic liquids and derived aerosols. The method involves protonation of nicotine to exploit use of FT-ICR-MS for nicotine quantification. The kinetics of nicotine protonation was determined to be a first order reaction. The activation energy $\left(\mathrm{E}_{\mathrm{a}}\right)$ for nicotine protonation was found to be $30.05 \mathrm{kJmol}^{-1}$. The measured nicotine levels of commercial e-liquids were within a difference range of $-2.94 \%$ to $25.20 \%$ from the manufacturer specified values. Nicotine in aerosols linearly increased as the number of 
puffs increased. Nicotine in aerosols also linearly increases with the nicotine concentration in e-liquids at the same puff number.

Unlike NIOSH 2551 GC-MS method, nicotine analysis in e-liquids using FTICR-MS does not require solvent extraction. Aerosol sample collection involving connection of three impingers in series is more efficient than NIOSH 2551 sorbent tubes. Also, analysis is of both the e-liquids and derived aerosol is easier, faster, and overall this method is more suitable for e-liquid aerosols than NIOSH 2551 method.

\subsection{EXPERIMENTAL SECTION}

\subsubsection{Experimental materials}

One popular electronic cigarette, blu plus ${ }^{\circledR}$ battery with two different flavored blu plus ${ }^{\circledR}$ cartridges (Classic Tobacco, Magnificent Menthol), was selected for this study. Ten popular e-liquids in the US market: three different flavored Halo e-liquids, two NJOY e-liquids, two VaporFi e-liquids with different levels of nicotine, an eVo Black diamond e-liquid, a Perfected Vapes, Clearwater e-liquid, and a Smooththol Nic Quid e-liquid, were also purchased. Table 1 lists detailed information of these e-liquids and cartridges. 37\% Hydrochloric acid, nicotine, and methanol were acquired from Sigma Aldrich. Deuterated nicotine was purchased from CDN Isotopes Inc. XRD-4 sorbent tubes for trapping nicotine were purchased from SKC company. The ultra-pure water used in this study was prepared using a Milli-Q water purification system (Millipore Corp., Bedford, MA, USA). 


\subsubsection{FT-ICR-MS}

An FT-ICR-MS instrument (Finnigan LTQ-FT, Thermo Electron, Bremen, Germany) equipped with a TriVersa NanoMate ion source (Advion BioSciences, Ithaca, NY) fitted with an electrospray chip (nozzle inner diameter $5.5 \mu \mathrm{m}$ ) was used for all mass spectral analyses. The TriVersa NanoMate was operated in positive ion mode by applying $2.0 \mathrm{kV}$ with no head pressure. Initially, lowresolution MS scans were acquired for 1 min to ensure the stability of ionization, after which high mass accuracy data were collected using the FT-ICR analyzer, where MS scans were acquired for $5 \mathrm{~min}$ and at the target mass resolution of 100,000 at $200 \mathrm{~m} / \mathrm{z}$.

\subsubsection{GC-MS analysis of nicotine}

An Agilent Technologies GC instrument equipped with a G4513A automatic sampler, Agilent 5975 series Mass Selective Detector in Electron Impact a TRACE 1310 GC with a split/splitless injector The GC had an Agilent 190915-433 column (30 m × $0.25 \mathrm{~mm} \times 0.25 \mu \mathrm{m}$ film thickness) Carrier gas helium flow rate was $1.5 \mathrm{~mL} / \mathrm{min}$. Column temperature was $55^{\circ} \mathrm{C}$ for $4 \mathrm{~min}$, then increased by $55^{\circ} \mathrm{C} / \mathrm{min}$ up to $220^{\circ} \mathrm{C}$ and was held at $220^{\circ} \mathrm{C}$ for $3 \mathrm{~min}$. The total running time was $10 \mathrm{~min}$. The samples were split injected with split flow of $100 \mathrm{~mL} / \mathrm{min}$ and a split ratio of 10 to 1 .

\subsubsection{Measurement of nicotine NMR spectroscopy}

To prepare a calibration curve for measurement of nicotine using ${ }^{1} \mathrm{H}$ NMR spectroscopy, six solutions containing different amounts of nicotine dissolved in $400 \mu \mathrm{L}$ DMSO- $d_{6}$ were prepared. The concentrations of nicotine from solution 1 
to solution 6 were set at $0.78,1.56,3.11,7.78,15.55$, and $38.88 \mu \mathrm{mol} / \mathrm{mL}$, respectively. Benzene was added to each solution as an internal reference at a concentration of $56.11 \mu \mathrm{mol} / \mathrm{mL} .{ }^{1} \mathrm{H}$ NMR spectra were obtained using a Varian 7600-AS instrument (400 MHz for $\left.{ }^{1} \mathrm{H}\right)$. The integration of the benzene hydrogen signal at $\delta 7.37 \mathrm{ppm}$ was set at a constant 6 , while the integrations of the nicotine protons between $\delta 8.45-8.49 \mathrm{ppm}$ were recorded. A linear calibration curve was was built and Fig. 3.2 shows a good linear dependence of $n$ (nicotine)/n(benzene) on $\mathrm{H}($ nicotine $) / \mathrm{H}($ benzene), where $\mathrm{n}=$ moles and $\mathrm{H}=$ corresponding proton integration of NMR spectra. We used this plot as an NMR calibration curve to measure nicotine concentration in the following kinetics study of nicotine protonation

\subsubsection{Kinetics measurement of nicotine protonation}

A kinetics study on the protonation of nicotine was conducted by measuring the amount of nicotine at different reaction times at reaction temperatures of $0,22,40$, and 60 ${ }^{\circ} \mathrm{C}$ using ${ }^{1} \mathrm{H}$ NMR. To a solution of nicotine $(5.0 \mu \mathrm{L}, 31.1 \mu \mathrm{mol})$ in water $(291 \mu \mathrm{L})$ at a given temperature, $37 \% \mathrm{HCl}(3.8 \mu \mathrm{L}, 37.4 \mu \mathrm{mol})$ was added. The mixture was magnetically stirred for a specified time. The reaction was terminated at a set time by addition of ethyl acetate $(400 \mu \mathrm{L})$ to extract any unprotonated nicotine. The organic layer then was separated from the aqueous layer with the aid of a pasture pipette and evaporated under vacuum. DMSO-d6 $(400 \mu \mathrm{L})$ was added to the extracted neutral nicotine and the solution was analyzed by ${ }^{1} \mathrm{H}$ NMR using benzene as the internal reference for quantification. 


\subsubsection{Analysis of nicotine in e-liquids}

Typically, $10 \mu \mathrm{L}$ of each e-liquid was added to a solution of $37 \% \mathrm{HCl}(5.0 \mu \mathrm{L}$, $49.2 \mu \mathrm{mol})$ in a mixture of $\mathrm{MeOH}(457.5 \mu \mathrm{L})$ and water $(37.5 \mu \mathrm{L})$ at room temperature. The reaction mixture was stirred for a minimum of 30 minutes to protonate nicotine. Then, $10 \mu \mathrm{L}$ of the reaction solution was removed and directly analyzed, in triplicate, by FT-ICR-MS. The measured nicotine was expressed as the average $[ \pm \mathrm{SD}]$ values.

\subsubsection{Collection and analysis of nicotine in e-cigarette aerosols}

A software-controlled (FlexiWare) cigarette-smoking robot (CSR) (Sci-Req, Montreal, CAN) was used to generate aerosols from blu plus ${ }^{\circledR}$ electronic cigarette battery (fixed voltage $3.7 \mathrm{~V}$ ) with e-liquids in the refillable mystic ${ }^{\circledR}$ cartridges. The puffing topography of e-cigarette users has been intensively studied. ${ }^{114-115}$ The mean puff duration, puff flow rate, and puff volume varied significantly among the subjects. The puffing protocol in this work consisted of 4 seconds of puff durations, $91.1 \mathrm{~mL}$ of puff volumes, and 26 seconds of puff intervals to closely mirror typical puffing topography of

e-cigarette users. ${ }^{114-115}$ Aerosols generated by the smoking robot flowed through a series of three impingers as shown in Figure 1 of the schematic diagram. Each impinger was charged with a solvent mixture of $\mathrm{MeOH}(91.5 \mathrm{~mL})$, water $(7.5 \mathrm{~mL})$, and $37 \% \mathrm{HCl}(1$ $\mathrm{mL}$ ). Initial experiments for collection of the aerosols with a series of four impingers indicated no nicotine in the fourth impinger. These experiments verified that the use of three impingers was sufficient for collection of all the aerosolized nicotine. After collection, $3 \mathrm{~mL}$ nicotinium-d3 was added to $500 \mathrm{~mL}$ of the solution from each impinger as an internal standard. The solution was then analyzed using FT-ICR-MS. To compare 
this collection of nicotine in aerosls with standard sorbent adsorption method (NIOSH 2551), nicotine in e-cigarette aerosols was also collected using XRD-4 sorbent tubes. NIOSH 2551 instruction was followed for collection of nicotine by the sorbent tubes, desorbing nicotine from the sorbent in ethyl acetate solution and analysis by GC-MS. 


\title{
CHAPTER 5
}

\section{SUMMARY AND FUTURE WORK}

\author{
5.1. SUMMARY \\ 5.2. FUTURE DIRECTION
}




\subsection{SUMMARY AND FUTURE WORK}

This research focused on using a silicon-based microreactor coated with derivatization reagents combined with either FT-ICR-MS or GC-MS for chemoselective capture, derivatization and analysis of trace level aldehydes and ketones. Sample sources included electronic cigarette aerosols, tobacco cigarettes and human expired breath. The quantification of nicotine in e-cig liquids and derived aerosols was also studied using an impinger set up.

\subsection{Summary}

We have used our powerful silicon microreactor coated with the aminooxy reagent $\mathrm{AMAH}$ as a derivatization reagent (coating) to capture and derivatize carbonyl compounds generated during aerosolization of electronic liquids. The oximation reaction of the functional cationic aminooxy compounds shown below with gaseous carbonyl species is a significant part of this dissertation.

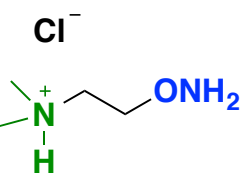

ADMH

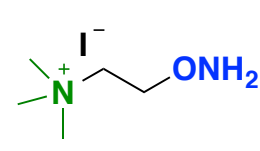

ATM

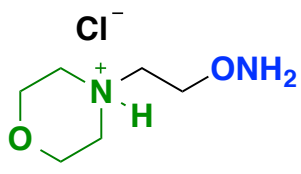

AMAH

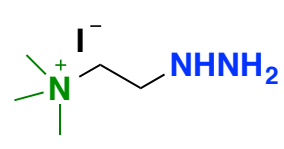

HTM

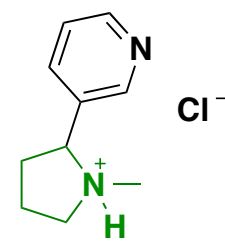

Nicotinium salt

Figure 5.0. ATM, ADMH, AMAH, HTM and nicotinium chloride salt

The installation of quaternary ammonium functional group on aminooxy (ADMH, ATM, and AMAH) and hydrazine (HTM) reagents, as well as nicotine analyte (Figure 5.0), enabled electrospray ionization efficiency of FT-ICR-MS. The aminooxy and hydrazine functional groups enabled chemoselective derivatization of the carbonyl compounds. We 
have used AMAH extensively to analyze carbonyl compounds in tobacco and electronic cigarettes.

We have demonstrated that the aerosolization of neat propylene glycol (PG) generated the highest level of acetaldehyde followed by formaldehyde and acetone. Hydroxyacetone was also produced. A low level of crotonaldehyde was also generated and this may have been produced from the aldol condensation of two molecules of acetaldehyde. This is the first time crotonaldehyde is reported in aerosol of neat propylene glycol. No acrolein was detected in neat PG. On the other hand neat glycerol (VG) also produced highest level of acetaldehyde followed by formaldehyde, acetone, and acrolein. No crotonaldehyde was produced. The use of a silicon-based microreactor for preconcentration of these trace-level carbonyl compounds in e-liquid aerosols and subsequent analysis with FT-ICM-MS represent a major contribution to the on-going research in analysis of carbonyl compounds in electronic cigarettes.

The carbonyl compounds produced during vaping of e-liquids increased as the vaping power increased. Consequently, the amount of carbonyl compounds detected in electronic cigarette aerosols increased as the vaping power (which is a function of coil resistance and vaping voltage) increased.

We also developed HTM to derivatize carbonyl compounds in expired breath using the silicon-based microreactor. This hydrazine salt has higher capture efficiency for $\alpha, \beta$-unsaturated aldehydes than its aminooxy analog ATM. Consistent with this observation, HTM activation energies are also lower with $\alpha, \beta$-unsaturated aldehydes than those of ATM.

We have also regioselectively protonated the pyrrolidine nitrogen of nicotine with 
an aqueous inorganic acid to form nicotinium chloride, which then was quantified with FT-ICR-MS. This approach solved most of the problems associated with the conventional standard nicotine method of analysis. Protonation of nicotine in both e-liquids and their derived aerosols facilitates effective and quantitative analysis by FT-ICR-MS.

NIOSH method utilizes a sorbent tube for nicotine sample collection and GC for analysis using quinoline as internal standard. The sorbent tube is not particularly suitable for collecting nicotine in e-liquid aerosols because these tubes have limited sample capacity. This limitation makes collection of aerosol samples where over 40 puffs need to be delivered, difficult. The use of impinger traps in this research mitigated this problem. The results of this research indicated that nicotine collected by the sorbent tube method were consistently lower than that collected by the impinger method. The lower values of the NIOSH method were induced by the escape of nicotine from the sorbent tube and incomplete desorption of nicotine from the sorbent. Also, NIOSH method involved extracting nicotine several times and this resulted in long sample preparation and consumption of high volume of solvent. Our new method did not involve nicotine extraction but direct infusion mass spectrometry. Therefore solvent volume and analysis time were significantly reduced. This impinger method of nicotine collection in e-liquid aerosols and analysis by FT-ICR-MS is therefore a remarkable novel contribution to nicotine analysis research.

\subsection{Future directions}

Our initial results supported the formation of carbonyl compounds from thermal decomposition of humectants. The contribution of thermal decomposition of other eliquid additives to the generation of these carbonyl compounds during aerosolization 
needs to be investigated. For instance, whereas nicotine has been known to generate highly carcinogenic 4-(methylnitrosamino)1-(3-pyridyl)-1-butanone (NNK) as well as highly reactive 4-(methylnitrosamino)- 4-(3-pyridy) butanal (NNA) by oxidative Nnitrosation under ring opening of the pyrrolidine ring (Scheme 6.1$),{ }^{164}$ the food grade flavor agent, 2-methylbutyraldehyde, can undergo thermal degradation to produce formaldehyde, acrolein, and other aldehydes ${ }^{165}$ (Scheme 5.2). While some of these carbonyls like acrolein, acetaldehyde and formaldehyde are considered toxic; others like NNK and NNA are highly carcinogenic. It is therefore imperative to determine if and/or how much these flavoring agents used in formulating these e-liquids contribute to the generation of these carbonyl compounds. Experiments of this nature will require that each of these additives and flavorants be aerosolized separately and the aerosols analyzed for carbonyl compounds. Having known the concentration of the carbonyl compounds generated from aerosols of neat humectants, a known amount of each of these additives can then be added to the humectants separately in different ratio before aerosolization. The contribution of these additives and flavorants can therefore be estimated.

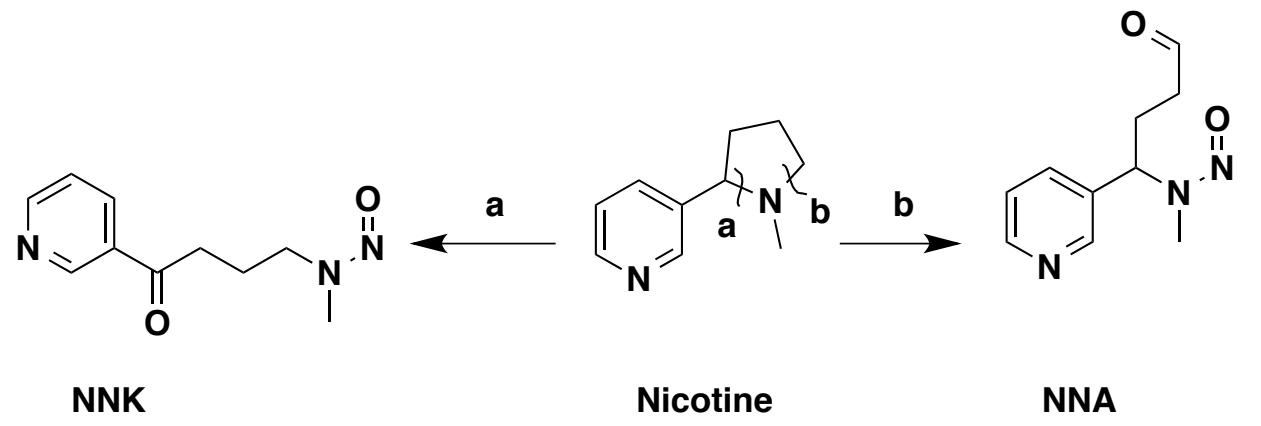

Scheme 5.0. Oxidative N-nitrosation of nicotine to form NNK and NNA. 


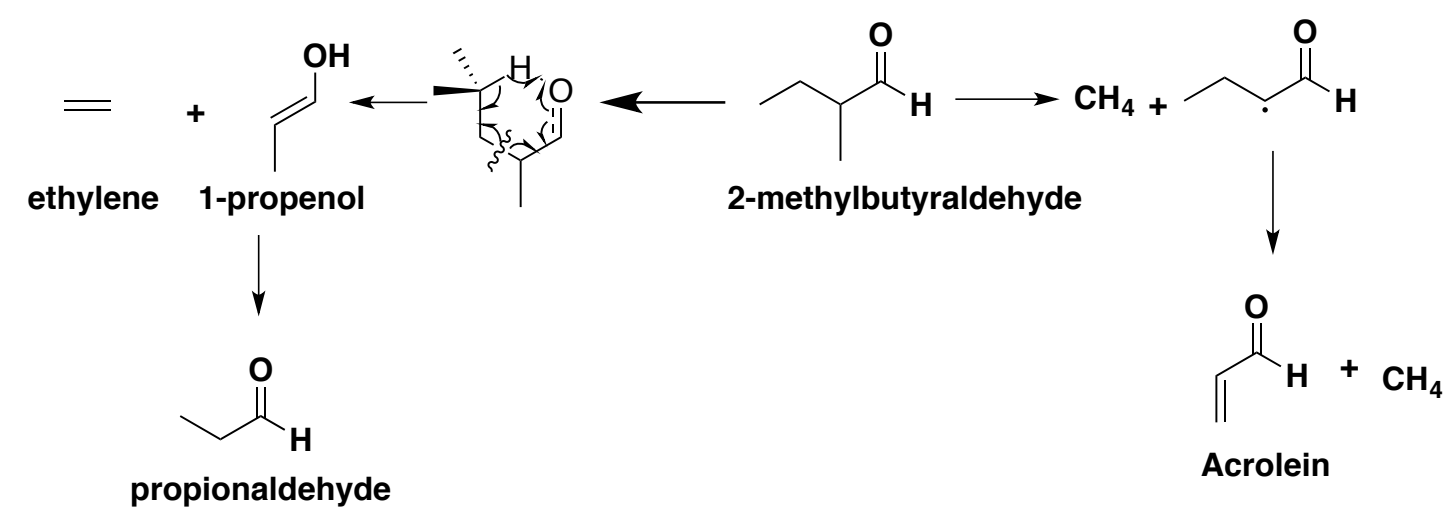

Scheme 5.1. Decomposition of 2-methylbutyraldehyde to generate acrolein and propionaldehyde.

$\alpha, \beta$-Unsaturated aldehydes, such as 4-hydroxyhexenal (4HHE) and 4hydroxyhexenal (4HNE), are known lung cancer biomarkers. Our research group has hitherto used ATM to analyze these unsaturated aldehydes for lung cancer diagnosis. Since all experimental results so far in this work have indicated that HTM is more reactive towards $\alpha, \beta$-unsaturated aldehydes than the other hydrazine reagents, the use of this novel hydrazine reagent to analyze 4HHE and 4HNE needs to be explored. This idea will require collaboration with medical personnel for collection and experimentation on expired breath samples of lung cancer patients.

A significant limitation in this research is the use of internal standard. We introduced an internal standard at the last step after the elution of the microreactor, prior to analysis using FT-ICR-MS or GC. This is because previous studies showed that more than $98 \%$ the acetone $d-6$ internal standard introduced to the tedlar bag was recovered at the end of evacuation of the Tedlar bag. ${ }^{54,129}$ Yet, an internal standard is best added at the very beginning of any analytical quantification studies including our own. This is to compensate for variations in sample preparation and instrument variation. Since we added it at the very end, it only compensated for instrument variation. Also, this research 
can be greatly improved by using isotopically labeled version of each molecule to be quantified in a multiplex quantification. That means each analyte should have its own isotopically labeled internal standard for excellent quantification. Also, the recovery of an analyte in an analytical sample should be performed. It is the detector response obtained from an amount of the analyte added to and extracted from the matrix, compared to the detector response for the true concentration of the pure authentic standard (seized materials). Although this has been established in form of capture efficiency of the system but this should be periodically reassessed. Recovery of the analyte in a sample needs not to be $100 \%$. However, recovery extent of both the internal standard and the analyte needs to be consistent and reproducible.

Another limitation of this research is that an aminooxy or a hydrazine-coated microchip can only identify/detect the carbonyl compounds components of analytical samples with accurate chemical formula, it cannot identify other components of the sample like dienes, furans, alcohols, ether, thiols etc. This limitation can be circumvented by incorporation of other chemoselective reagents into the microreactor. For example, a very powerful dienophile can derivatize cis-1, 3-dienes. 


\section{REFERENCES}

1. Sakuragawa, A.; Yoneno, T.; Inoue, K.; Okutani, T., Trace analysis of carbonyl compounds by liquid chromatography-mass spectrometry after collection as 2,4dinitrophenylhydrazine derivatives. Journal of Chromatography A 1999, 844 (12), 403-408.

2. Kölliker, S.; Oehme, M.; Dye, C., Structure Elucidation of 2,4Dinitrophenylhydrazone Derivatives of Carbonyl Compounds in Ambient Air by HPLC/MS and Multiple MS/MS Using Atmospheric Chemical Ionization in the Negative Ion Mode. Analytical Chemistry 1998, 70 (9), 1979-1985.

3. Grekov, A. P.; Veselov, V. Y., The $\alpha$-Effect in the Chemistry of Organic Compounds. Russian Chemical Reviews 1978, 47 (7), 631.

4. Kool, E. T.; Crisalli, P.; Chan, K. M., Fast Alpha Nucleophiles: Structures that Undergo Rapid Hydrazone/Oxime Formation at Neutral pH. Organic Letters 2014, 16 (5), 1454-1457.

5. Jjunju, F. P. M.; Li, A.; Badu-Tawiah, A.; Wei, P.; Li, L.; Ouyang, Z.; Roqan, I. S.; Cooks, R. G., In situ analysis of corrosion inhibitors using a portable mass spectrometer with paper spray ionization. Analyst 2013, 138 (13), 3740-3748.

6. Haussmann, H. J., Use of hazard indices for a theoretical evaluation of cigarette smoke composition. Chem Res Toxicol 2012, 25 (4), 794-810.

7. Conklin, D. J.; Barski, O. A.; Lesgards, J. F.; Juvan, P.; Rezen, T.; Rozman, D.; Prough, R. A.; Vladykovskaya, E.; Liu, S.; Srivastava, S.; Bhatnagar, A., Acrolein consumption induces systemic dyslipidemia and lipoprotein modification. Toxicol Appl Pharmacol 2010, 243 (1), 1-12.

8. Conklin, D. J.; Bhatnagar, A.; Cowley, H. R.; Johnson, G. H.; Wiechmann, R. J.; Sayre, L. M.; Trent, M. B.; Boor, P. J., Acrolein generation stimulates hypercontraction in isolated human blood vessels. Toxicology and applied pharmacology 2006, 217 (3), 277-288.

9. Conklin, D. J.; Haberzettl, P.; Prough, R. A.; Bhatnagar, A., Glutathione-Stransferase $\mathrm{P}$ protects against endothelial dysfunction induced by exposure to tobacco smoke. American journal of physiology. Heart and circulatory physiology 2009, 296 (5), H1586-97.

10. Sithu, S. D.; Srivastava, S.; Siddiqui, M. A.; Vladykovskaya, E.; Riggs, D. W.; Conklin, D. J.; Haberzettl, P.; O'Toole, T. E.; Bhatnagar, A.; D'Souza, S. E., Exposure to acrolein by inhalation causes platelet activation. Toxicol Appl Pharmacol 2010, 248 (2), 100-10.

11. Srivastava, S.; Sithu, S. D.; Vladykovskaya, E.; Haberzettl, P.; Hoetker, D. J.; Siddiqui, M. A.; Conklin, D. J.; D'Souza, S. E.; Bhatnagar, A., Oral exposure to acrolein exacerbates atherosclerosis in apoE-null mice. Atherosclerosis 2011, 215 (2), 301-8. 
12. O'Toole, T. E.; Zheng, Y. T.; Hellmann, J.; Conklin, D. J.; Barski, O.; Bhatnagar, A., Acrolein activates matrix metalloproteinases by increasing reactive oxygen species in macrophages. Toxicol Appl Pharmacol 2009, 236 (2), 194-201.

13. Wang, G.-W.; Guo, Y.; Vondriska, T. M.; Zhang, J.; Zhang, S.; Tsai, L. L.; Zong, N. C.; Bolli, R.; Bhatnagar, A.; Prabhu, S. D., Acrolein consumption exacerbates myocardial ischemic injury and blocks nitric oxide-induced $\mathrm{PKC} \varepsilon$ signaling and cardioprotection. Journal of Molecular and Cellular Cardiology 2008, 44 (6), 1016-1022.

14. Ismahil, M. A.; Hamid, T.; Haberzettl, P.; Gu, Y.; Chandrasekar, B.; Srivastava, S.; Bhatnagar, A.; Prabhu, S. D., Chronic oral exposure to the aldehyde pollutant acrolein induces dilated cardiomyopathy. American journal of physiology. Heart and circulatory physiology 2011, 301 (5), H2050-60.

15. DeJarnett, N.; Conklin, D. J.; Riggs, D. W.; Myers, J. A.; O'Toole, T. E.; Hamzeh, I.; Wagner, S.; Chugh, A.; Ramos, K. S.; Srivastava, S.; Higdon, D.; Tollerud, D. J.; DeFilippis, A.; Becher, C.; Wyatt, B.; McCracken, J.; Abplanalp, W.; Rai, S. N.; Ciszewski, T.; Xie, Z.; Yeager, R.; Prabhu, S. D.; Bhatnagar, A., Acrolein exposure is associated with increased cardiovascular disease risk. Journal of the American Heart Association 2014, 3 (4).

16. Riedel, K.; Weller, R.; Schrems, O., Variability of formaldehyde in the Antarctic troposphere. Physical Chemistry Chemical Physics 1999, 1 (24), 5523-5527.

17. Atkinson, R., Gas-phase tropospheric chemistry of organic compounds: a review. Atmospheric Environment 2007, 41 (Supplement), 200-240.

18. Kirchstetter, T. W.; Singer, B. C.; Harley, R. A.; Kendall, G. R.; Chan, W., Impact of Oxygenated Gasoline Use on California Light-Duty Vehicle Emissions. Environmental Science \& Technology 1996, 30 (2), 661-670.

19. Fraser, M. P.; Cass, G. R.; Simoneit, B. R. T.; Rasmussen, R. A., Air Quality Model Evaluation Data for Organics. 5. C6-C22 Nonpolar and Semipolar Aromatic Compounds. Environmental Science \& Technology 1998, 32 (12), 1760-1770.

20. Maejima, K.; Suzuki, T.; Niwa, K.; Numata, H.; Maekawa, A.; Nagase, S.; Ishinishi, N., Toxicity to rats of methanol-fueled engine exhaust inhaled continuously for 28 days. Journal of Toxicology and Environmental Health 1992, 37 (2), 293-312.

21. Zervas, E.; Montagne, X.; Lahaye, J., Emission of Alcohols and Carbonyl Compounds from a Spark Ignition Engine. Influence of Fuel and Air/Fuel Equivalence Ratio. Environmental Science \& Technology 2002, 36 (11), 24142421.

22. Grosjean, E.; Grosjean, D.; Fraser, M. P.; Cass, G. R., Air Quality Model Evaluation Data for Organics. 2. C1-C14 Carbonyls in Los Angeles Air. Environmental Science \& Technology 1996, 30 (9), 2687-2703.

23. Lu, X.; Cai, J.; Kong, H.; Wu, M.; Hua, R.; Zhao, M.; Liu, J.; Xu, G., Analysis of Cigarette Smoke Condensates by Comprehensive Two-Dimensional Gas Chromatography/Time-of-Flight Mass Spectrometry I Acidic Fraction. Analytical Chemistry 2003, 75 (17), 4441-4451. 
24. Caldwell, W. S.; Conner, J. M., Artifact formation during smoke trapping: an improved method for determination of $\mathrm{N}$-nitrosamines in cigarette smoke. Journal - Association of Official Analytical Chemists 1990, 73 (5), 783-9.

25. Hecht, S. S.; Ornaf, R. M.; Hoffmann, D., Determination of N'-nitrosonornicotine in tobacco by high speed liquid chromatography. Analytical Chemistry 1975, 47 (12), 2046-2048.

26. Muto, H.; Takizawa, Y., Dioxins in cigarette smoke. Archives of environmental health 1989, 44 (3), 171-4.

27. White, E. L.; Uhrig, M. S.; Johnson, T. J.; Gordon, B. M.; Hicks, R. D.; Borgerding, M. F.; Coleman, W. M., 3rd; Elder, J. F., Jr., Quantitative determination of selected compounds in a Kentucky 1R4F reference cigarette smoke by multidimensional gas chromatography and selected ion monitoringmass spectrometry. J Chromatogr Sci 1990, 28 (8), 393-9.

28. Miyake, T.; Shibamoto, T., Quantitative analysis of acetaldehyde in foods and beverages. Journal of Agricultural and Food Chemistry 1993, 41 (11), 19681970.

29. Horváth, I.; Lázár, Z.; Gyulai, N.; Kollai, M.; Losonczy, G., Exhaled biomarkers in lung cancer. European Respiratory Journal 2009, 34 (1), 261-275.

30. Wang, C.; Dong, R.; Wang, X.; Lian, A.; Chi, C.; Ke, C.; Guo, L.; Liu, S.; Zhao, $\mathrm{W}$; $\mathrm{Xu}, \mathrm{G} . ; \mathrm{Li}$, E., Exhaled volatile organic compounds as lung cancer biomarkers during one-lung ventilation. Scientific Reports 2014, 4, 7312.

31. Fu, X.-A.; Li, M.; Knipp, R. J.; Nantz, M. H.; Bousamra, M., Noninvasive detection of lung cancer using exhaled breath. Cancer Medicine 2014, 3 (1), 174181.

32. Jin, H.; Zangar, R. C., Protein Modifications as Potential Biomarkers in Breast Cancer. Biomarker Insights 2009, 4 (1761-BMI-Protein-Modifications-asPotential-Biomarkers-in-Breast-Cancer.pdf), 191-200.

33. Albright, C. D.; Klem, E.; Shah, A. A.; Gallagher, P., Breast cancer cell-targeted oxidative stress: Enhancement of cancer cell uptake of conjugated linoleic acid, activation of p53, and inhibition of proliferation. Experimental and Molecular Pathology 2005, 79 (2), 118-125.

34. Yong, P. H.; Zong, H.; Medina, R. J.; Limb, G. A.; Uchida, K.; Stitt, A. W.; Curtis, T. M., Evidence supporting a role for $\mathrm{N}(\varepsilon)$-(3-formyl-3,4dehydropiperidino)lysine accumulation in Müller glia dysfunction and death in diabetic retinopathy. Molecular Vision 2010, 16, 2524-2538.

35. Cao, W.; Duan, Y., Breath Analysis: Potential for Clinical Diagnosis and Exposure Assessment. Clinical Chemistry 2006, 52 (5), 800-811.

36. Kim, K.-H., Some Insights into the Gas Chromatographic Determination of Reduced Sulfur Compounds (RSCs) in Air. Environmental Science \& Technology 2005, 39 (17), 6765-6769.

37. Dettmer, K.; Engewald, W., Adsorbent materials commonly used in air analysis for adsorptive enrichment and thermal desorption of volatile organic compounds. Analytical and Bioanalytical Chemistry 2002, 373 (6), 490-500.

38. Costa, R., Newly Introduced Sample Preparation Techniques: Towards Miniaturization. Critical Reviews in Analytical Chemistry 2014, 44 (4), 299-310. 
39. Arthur, C. L.; Pawliszyn, J., Solid phase microextraction with thermal desorption using fused silica optical fibers. Analytical Chemistry 1990, 62 (19), 2145-2148.

40. Vas, G.; Vékey, K., Solid-phase microextraction: a powerful sample preparation tool prior to mass spectrometric analysis. Journal of Mass Spectrometry 2004, 39 (3), 233-254.

41. Zaitsev, V. N.; Zui, M. F., Preconcentration by solid-phase microextraction. Journal of Analytical Chemistry 2014, 69 (8), 715-727.

42. Hyötyläinen, T.; Riekkola, M.-L., Sorbent- and liquid-phase microextraction techniques and membrane-assisted extraction in combination with gas chromatographic analysis: A review. Analytica Chimica Acta 2008, 614 (1), $27-$ 37.

43. Kataoka, H.; Lord, H. L.; Pawliszyn, J., Applications of solid-phase microextraction in food analysis. Journal of Chromatography A 2000, 880 (1), $35-62$.

44. Kaur, V.; Malik, A. K.; Verma, N., Applications of solid phase microextraction for the determination of metallic and organometallic species. Journal of Separation Science 2006, 29 (3), 333-345.

45. Lin, C.-C.; Hsu, J.-L.; Lee, G.-B., Sample preconcentration in microfluidic devices. Microfluidics and Nanofluidics 2011, 10 (3), 481-511.

46. Bassam Alfeeli, a. M. A., MEMS-Based Selective Preconcentration of Trace Level Breath Analytes. IEEE Sensors Journal 9 (9), 1068-1075.

47. Fu, X.-A.; Li, M.; Biswas, S.; Nantz, M. H.; Higashi, R. M., A novel microreactor approach for analysis of ketones and aldehydes in breath. The Analyst 2011, 136 (22), 4662-4666.

48. Vogel, M.; Büldt, A.; Karst, U., Hydrazine reagents as derivatizing agents in environmental analysis - a critical review. Fresenius' Journal of Analytical Chemistry 2000, 366 (8), 781-791.

49. Zwiener, C.; Glauner, T.; Frimmel, F., Method optimization for the determination of carbonyl compounds in disinfected water by DNPH derivatization and LCESI-MS-MS. Analytical and Bioanalytical Chemistry 2002, 372 (5), 615-621.

50. Tejada, S. B., Evaluation of Silica Gel Cartridges Coated In Situ with Acidified 2, 4-Dinitrophenylhydrazine for Sampling Aldehydes and Ketones in Air. International Journal of Environmental Analytical Chemistry 1986, 26 (2), 167185.

51. He, C.; Ge, Y.; Tan, J.; You, K.; Han, X.; Wang, J.; You, Q.; Shah, A. N., Comparison of carbonyl compounds emissions from diesel engine fueled with biodiesel and diesel. Atmospheric Environment 2009, 43 (24), 3657-3661.

52. Szulejko, J. E.; Kim, K.-H., Derivatization techniques for determination of carbonyls in air. TrAC Trends in Analytical Chemistry 2015, 64, 29-41.

53. Iwasaki, Y.; Nakano, Y.; Mochizuki, K.; Nomoto, M.; Takahashi, Y.; Ito, R.; Saito, K.; Nakazawa, H., A new strategy for ionization enhancement by derivatization for mass spectrometry. Journal of Chromatography B 2011, 879 (17-18), 1159-1165.

54. Li, M.; Biswas, S.; Nantz, M. H.; Higashi, R. M.; Fu, X.-A., Preconcentration and Analysis of Trace Volatile Carbonyl Compounds. Analytical Chemistry 2012, 84 (3), 1288-1293. 
55. Knipp, R. J.; Li, M.; Fu, X.; Nantz, M. H., A Versatile Probe for Chemoselective Capture and Analysis of Carbonyl Compounds in Exhaled Breath. Analytical Methods 2015.

56. Johnson, D. W., A modified Girard derivatizing reagent for universal profiling and trace analysis of aldehydes and ketones by electrospray ionization tandem mass spectrometry. Rapid Communications in Mass Spectrometry 2007, 21 (18), 2926-2932.

57. Leaf-nosed bat. In Encyclopeedia Britannica, Encyclopædia Britannica Online: 2009.

58. Li, M. A microreactor approach for chemoselective capture and analysis of carbonyl compounds in air and exhaled breath. 2013. https://doi.org/10.18297/etd/826.

59. Herrington, J. S.; Myers, C., Electronic cigarette solutions and resultant aerosol profiles. Journal of Chromatography A 2015, 1418 (Supplement C), 192-199.

60. Phillips, M.; Cataneo, R. N.; Chaturvedi, A.; Kaplan, P. D.; Libardoni, M.; Mundada, M.; Patel, U.; Zhang, X., Detection of an extended human volatome with comprehensive two-dimensional gas chromatography time-of-flight mass spectrometry. PLoS One 2013, 8 (9), e75274.

61. Mondello, L.; Tranchida, P. Q.; Dugo, P.; Dugo, G., Comprehensive twodimensional gas chromatography-mass spectrometry: a review. Mass Spectrom Rev 2008, 27 (2), 101-24.

62. Flora, J. W.; Wilkinson, C. T.; Wilkinson, J. W.; Lipowicz, P. J.; Skapars, J. A.; Anderson, A.; Miller, J. H., Method for the Determination of Carbonyl Compounds in E-Cigarette Aerosols. Journal of Chromatographic Science 2017, 55 (2), 142-148.

63. Westhoff, M.; Litterst, P.; Freitag, L.; Urfer, W.; Bader, S.; Baumbach, J. I., Ion mobility spectrometry for the detection of volatile organic compounds in exhaled breath of patients with lung cancer: results of a pilot study. Thorax 2009, 64 (9), 744-8.

64. Agnieszka Ulanowska, M. L., Anton Amann and Boguslaw Buszewski, Determination of Volatile Organic Compounds in Exhaled Breath by Ion Mobility Spectrometry. Chem. Anal. (Warsaw) 2008, 53, 953.

65. Fleischer, M.; Simon, E.; Rumpel, E.; Ulmer, H.; Harbeck, M.; Wandel, M.; Fietzek, C.; Weimar, U.; Meixner, H., Detection of volatile compounds correlated to human diseases through breath analysis with chemical sensors. Sensors and Actuators B: Chemical 2002, 83 (1), 245-249.

66. Chapman, E. A.; Thomas, P. S.; Stone, E.; Lewis, C.; Yates, D. H., A breath test for malignant mesothelioma using an electronic nose. The European respiratory journal 2012, 40 (2), 448-54.

67. Fernandes, M. P.; Venkatesh, S.; Sudarshan, B. G., Early Detection of Lung Cancer Using Nano-Nose - A Review. The open biomedical engineering journal 2015, 9, 228-33.

68. Mark, S.; Petia, A. S.; Haiying, Z.; Bethanne, M. W., Utility of the Hybrid LTQFTMS for Drug Metabolism Applications. Current Drug Metabolism 2006, 7 (5), 547-555. 
69. Mattingly, S. J.; Xu, T.; Nantz, M. H.; Higashi, R. M.; Fan, T. W., A Carbonyl Capture Approach for Profiling Oxidized Metabolites in Cell Extracts. Metabolomics : Official journal of the Metabolomic Society 2012, 8 (6), 989-996.

70. Somogyi, Á.; Thissen, R.; Orthous-Daunay, F.-R.; Vuitton, V., The Role of Ultrahigh Resolution Fourier Transform Mass Spectrometry (FT-MS) in Astrobiology-Related Research: Analysis of Meteorites and Tholins. International Journal of Molecular Sciences 2016, 17 (4), 439.

71. Jensen, R. P.; Luo, W.; Pankow, J. F.; Strongin, R. M.; Peyton, D. H., Hidden Formaldehyde in E-Cigarette Aerosols. New England Journal of Medicine 2015, 372 (4), 392-394.

72. Bekki, K.; Uchiyama, S.; Ohta, K.; Inaba, Y.; Nakagome, H.; Kunugita, N., Carbonyl Compounds Generated from Electronic Cigarettes. International Journal of Environmental Research and Public Health 2014, 11 (11), 11192.

73. Ogunwale, M. A.; Chen, Y.; Theis, W. S.; Nantz, M. H.; Conklin, D. J.; Fu, X.A., A novel method of nicotine quantification in electronic cigarette liquids and aerosols. Analytical Methods 2017, 9 (29), 4261-4266.

74. Hon, L., Electronic cigarette. Google Patents: 2009.

75. Hon, L., Electronic atomization cigarette. Google Patents: 2010.

76. Grana, R.; Benowitz, N.; Glantz, S. A., E-Cigarettes. A Scientific Review 2014, 129 (19), 1972-1986.

77. Cheng, T., Chemical evaluation of electronic cigarettes. Tobacco Control 2014, 23 (Suppl 2), ii11-ii17.

78. Erickson, B. E., Boom In E-Cigarettes Sparks Calls For Regulation. C\&EN Chemical \& Engineering News 2015, 93 (7), 10-13.

79. Farsalinos, K. E.; Kistler, K. A.; Gillman, G.; Voudris, V., Evaluation of electronic cigarette liquids and aerosol for the presence of selected inhalation toxins. Nicotine \& tobacco research : official journal of the Society for Research on Nicotine and Tobacco 2015, 17 (2), 168-74.

80. Williams, M.; Villarreal, A.; Bozhilov, K.; Lin, S.; Talbot, P., Metal and Silicate Particles Including Nanoparticles Are Present in Electronic Cigarette Cartomizer Fluid and Aerosol. PLOS ONE 2013, 8 (3), e57987.

81. Franck, C.; Budlovsky, T.; Windle, S. B.; Filion, K. B.; Eisenberg, M. J., <span hwp:id="article-title-1" class="article-title" $>$ Electronic Cigarettes in North America $<$ span $><$ span hwp:id="article-title-40" class="sub-articletitle" $>$ CLINICAL PERSPECTIVE $</$ span $>$. History, Use, and Implications for Smoking Cessation 2014, 129 (19), 1945-1952.

82. Goniewicz, M. L.; Kuma, T.; Gawron, M.; Knysak, J.; Kosmider, L., Nicotine Levels in Electronic Cigarettes. Nicotine \& Tobacco Research 2013, 15 (1), 158166.

83. M. Alvarado, S. K., A. Kumar, C. P. Osborne, P. J. Solomon Electronic Cigarettes: Secondary Research Overview. (accessed September 27).

84. Gostin, L. O.; Glasner, A. Y., E-cigarettes, vaping, and youth. JAMA 2014, 312 (6), 595-596.

85. Leventhal, A. M.; Strong, D. R.; Kirkpatrick, M. G.; et al., Association of electronic cigarette use with initiation of combustible tobacco product smoking in early adolescence. JAMA 2015, 314 (7), 700-707. 
86. Petersen, I. N.; Crestey, F.; Jensen, A. A.; Indurthi, D. C.; Pedersen, H.; Andreasen, J. T.; Balle, T.; Kristensen, J. L., Tying up Nicotine: New Selective Competitive Antagonist of the Neuronal Nicotinic Acetylcholine Receptors. ACS Medicinal Chemistry Letters 2015, 6 (4), 472-475.

87. Templeton, D. Three men suffer serious burns from e-cigarette battery fires. http://www.post-gazette.com/local/region/2016/03/18/Three-men-suffer-seriousburns-from-e-cigarette-battery-fires/stories/201603170020 (accessed 21

November).

88. Siegel, M. B.; Tanwar, K. L.; Wood, K. S., Electronic Cigarettes As a SmokingCessation Tool: Results from an Online Survey. American Journal of Preventive Medicine 2011, 40 (4), 472-475.

89. Ghilarducci, D. P.; Tjeerdema, R. S., Fate and effects of acrolein. Reviews of environmental contamination and toxicology 1995, 144, 95-146.

90. Dong, J.-Z.; Moldoveanu, S. C., Gas chromatography-mass spectrometry of carbonyl compounds in cigarette mainstream smoke after derivatization with 2,4dinitrophenylhydrazine. Journal of Chromatography A 2004, 1027 (1-2), 25-35.

91. Stepanov, I.; Jensen, J.; Hatsukami, D.; Hecht, S. S., New and traditional smokeless tobacco: comparison of toxicant and carcinogen levels. Nicotine \& tobacco research : official journal of the Society for Research on Nicotine and Tobacco 2008, 10 (12), 1773-82.

92. deBethizy, J. D.; Borgerding, M. F.; Doolittle, D. J.; Robinson, J. H.; McManus, K. T.; Rahn, C. A.; Davis, R. A.; Burger, G. T.; Hayes, J. R.; Reynolds, J. H. t.; et al., Chemical and biological studies of a cigarette that heats rather than burns tobacco. Journal of clinical pharmacology 1990, 30 (8), 755-63.

93. Shihadeh, A.; Salman, R.; Jaroudi, E.; Saliba, N.; Sepetdjian, E.; Blank, M. D.; Cobb, C. O.; Eissenberg, T., Does switching to a tobacco-free waterpipe product reduce toxicant intake? A crossover study comparing $\mathrm{CO}, \mathrm{NO}, \mathrm{PAH}$, volatile aldehydes, "tar" and nicotine yields. Food and chemical toxicology : an international journal published for the British Industrial Biological Research Association 2012, 50 (5), 1494-8.

94. Stanfill, S. B.; Calafat, A. M.; Brown, C. R.; Polzin, G. M.; Chiang, J. M.; Watson, C. H.; Ashley, D. L., Concentrations of nine alkenylbenzenes, coumarin, piperonal and pulegone in Indian bidi cigarette tobacco. Food and Chemical Toxicology 2003, 41 (2), 303-317.

95. McAuley, T. R.; Hopke, P. K.; Zhao, J.; Babaian, S., Comparison of the effects of e-cigarette vapor and cigarette smoke on indoor air quality. Inhalation toxicology 2012, 24 (12), 850-7.

96. Goniewicz, M. L.; Knysak, J.; Gawron, M.; Kosmider, L.; Sobczak, A.; Kurek, J.; Prokopowicz, A.; Jablonska-Czapla, M.; Rosik-Dulewska, C.; Havel, C.; Jacob, P., 3rd; Benowitz, N., Levels of selected carcinogens and toxicants in vapour from electronic cigarettes. Tob Control 2014, 23 (2), 133-9.

97. Kosmider, L.; Sobczak, A.; Fik, M.; Knysak, J.; Zaciera, M.; Kurek, J.; Goniewicz, M. L., Carbonyl Compounds in Electronic Cigarette Vapors-Effects of Nicotine Solvent and Battery Output Voltage. Nicotine \& Tobacco Research 2014. 
98. Allen, J. G.; Flanigan, S. S.; LeBlanc, M.; Vallarino, J.; MacNaughton, P.; Stewart, J. H.; Christiani, D. C., Flavoring Chemicals in E-Cigarettes: Diacetyl, 2,3-Pentanedione, and Acetoin in a Sample of 51 Products, Including Fruit-, Candy-, and Cocktail-Flavored E-Cigarettes. Environ Health Perspect 2016, 124 (6), 733-9.

99. Fuoco, F. C.; Buonanno, G.; Stabile, L.; Vigo, P., Influential parameters on particle concentration and size distribution in the mainstream of e-cigarettes. Environmental pollution (Barking, Essex : 1987) 2014, 184, 523-9.

100. Ogunwale, M. A.; Li, M.; Ramakrishnam Raju, M. V.; Chen, Y.; Nantz, M. H.; Conklin, D. J.; Fu, X.-A., Aldehyde Detection in Electronic Cigarette Aerosols. ACS Omega 2017, 2 (3), 1207-1214.

101. Klager, S.; Vallarino, J.; MacNaughton, P.; Christiani, D. C.; Lu, Q.; Allen, J. G., Flavoring Chemicals and Aldehydes in E-Cigarette Emissions. Environmental Science \& Technology 2017, 51 (18), 10806-10813.

102. Talih, S.; Balhas, Z.; Eissenberg, T.; Salman, R.; Karaoghlanian, N.; El Hellani, A.; Baalbaki, R.; Saliba, N.; Shihadeh, A., Effects of user puff topography, device voltage, and liquid nicotine concentration on electronic cigarette nicotine yield: measurements and model predictions. Nicotine \& tobacco research : official journal of the Society for Research on Nicotine and Tobacco 2015, 17 (2), 150-7.

103. Shihadeh, A.; Talih, S.; Eissenberg, T., Commentary on Farsalinos et al. (2015): E-cigarettes generate high levels of aldehydes only in 'dry puff' conditions. Addiction 2015, 110 (11), 1861-1862.

104. Joel L. Nitzkin, K. F., Michael Siegel, More on Hidden Formaldehyde in ECigarette Aerosols. New England Journal of Medicine 2015, 372 (16), 1575-1577.

105. Shen, J.; Shan, W.; Zhang, Y.; Du, J.; Xu, H.; Fan, K.; Shen, W.; Tang, Y., A novel catalyst with high activity for polyhydric alcohol oxidation: nanosilver/zeolite film. Chemical communications (Cambridge, England) 2004, (24), 2880-1.

106. Shen, J.; Shan, W.; Zhang, Y.; Du, J.; Xu, H.; Fan, K.; Shen, W.; Tang, Y., Gasphase selective oxidation of alcohols: In situ electrolytic nano-silver/zeolite film/copper grid catalyst. Journal of Catalysis 2006, 237 (1), 94-101.

107. Díaz, E.; Sad, M. E.; Iglesia, E., Homogeneous Oxidation Reactions of Propanediols at Low Temperatures. ChemSusChem 2010, 3 (9), 1063-1070.

108. Bayramoğlu, D.; Gürel, G.; Sinag, A.; Güllü, M., Thermal conversion of glycerol to value-added chemicals: Pyridine derivatives by one-pot microwave-assisted synthesis. 2014; Vol. 38, p 661-670.

109. Rennard, D. C.; Kruger, J. S.; Schmidt, L. D., Autothermal Catalytic Partial Oxidation of Glycerol to Syngas and to Non-Equilibrium Products. ChemSusChem 2009, 2 (1), 89-98.

110. Wang, W., Thermodynamic analysis of glycerol partial oxidation for hydrogen production. Fuel Processing Technology 2010, 91 (11), 1401-1408.

111. Lin, Y.-C., Catalytic valorization of glycerol to hydrogen and syngas. International Journal of Hydrogen Energy 2013, 38 (6), 2678-2700.

112. Balashov, A. L.; Danov, S. M.; Krasnov, V. L.; Chernov, A. Y.; Ryabova, T. A., Association of Formaldehyde in Aqueous-Alcoholic Systems. Russian Journal of General Chemistry 2002, 72 (5), 744-747. 
113. Chopade, S. P.; Sharma, M. M., Acetalization of ethylene glycol with formaldehyde using cation-exchange resins as catalysts: batch versus reactive distillation. Reactive and Functional Polymers 1997, 34 (1), 37-45.

114. Robinson, R. J.; Hensel, E. C.; Morabito, P. N.; Roundtree, K. A., Electronic Cigarette Topography in the Natural Environment. PLOS ONE 2015, 10 (6), e0129296.

115. Evans, S. E.; Hoffman, A. C., Electronic cigarettes: abuse liability, topography and subjective effects. Tobacco Control 2014, 23 (suppl 2), ii23-ii29.

116. Farsalinos, K. E.; Voudris, V.; Poulas, K., E-cigarettes generate high levels of aldehydes only in 'dry puff' conditions. Addiction 2015, 110 (8), 1352-1356.

117. Sleiman, M.; Logue, J. M.; Montesinos, V. N.; Russell, M. L.; Litter, M. I.; Gundel, L. A.; Destaillats, H., Emissions from Electronic Cigarettes: Key Parameters Affecting the Release of Harmful Chemicals. Environmental Science \& Technology 2016, 50 (17), 9644-9651.

118. Laino, T.; Tuma, C.; Curioni, A.; Jochnowitz, E.; Stolz, S., A Revisited Picture of the Mechanism of Glycerol Dehydration. The Journal of Physical Chemistry A 2011, 115 (15), 3592-3595.

119. Laino, T.; Tuma, C.; Moor, P.; Martin, E.; Stolz, S.; Curioni, A., Mechanisms of Propylene Glycol and Triacetin Pyrolysis. The Journal of Physical Chemistry A 2012, 116 (18), 4602-4609.

120. Hess, C. A.; Olmedo, P.; Navas-Acien, A.; Goessler, W.; Cohen, J. E.; Rule, A. M., E-cigarettes as a source of toxic and potentially carcinogenic metals. Environmental Research 2017, 152 (Supplement C), 221-225.

121. Farsalinos, K. E.; Voudris, V.; Poulas, K., Are Metals Emitted from Electronic Cigarettes a Reason for Health Concern? A Risk-Assessment Analysis of Currently Available Literature. International journal of environmental research and public health 2015, 12 (5), 5215-5232.

122. Walker, J. F.; Chadwick, A. F., Trioxane as a Source of Formaldehyde. Industrial \& Engineering Chemistry 1947, 39 (8), 974-977.

123. Kolb, H. C.; Finn, M. G.; Sharpless, K. B., Click Chemistry: Diverse Chemical Function from a Few Good Reactions. Angewandte Chemie International Edition 2001, 40 (11), 2004-2021.

124. Laulhe, S. Aminooxy reagents for synthesis and analysis : expanding the role of oximation Electronic Theses and Dissertations [Online], 2013. https://doi.org/10.18297/etd/796.

125. Mattingly, S.; Xu, T.; Nantz, M.; Higashi, R.; Fan, T. M., A carbonyl capture approach for profiling oxidized metabolites in cell extracts. Metabolomics 2012, 8 (6), 989-996.

126. Iglesias, J.; Gallardo, J. M.; Medina, I., Determination of carbonyl compounds in fish species samples with solid-phase microextraction with on-fibre derivatization. Food Chemistry 2010, 123 (3), 771-778.

127. Lees, A.; Sen, G.; LopezAcosta, A., Versatile and efficient synthesis of proteinpolysaccharide conjugate vaccines using aminooxy reagents and oxime chemistry. Vaccine 2006, 24 (6), 716-729. 
128. Hudak, J. E.; Yu, H. H.; Bertozzi, C. R., Protein Glycoengineering Enabled by the Versatile Synthesis of Aminooxy Glycans and the Genetically Encoded Aldehyde Tag. Journal of the American Chemical Society 2011, 133 (40), 16127-16135.

129. Li, M.; Biswas, S.; Nantz, M. H.; Higashi, R. M.; Fu, X.-A., A microfabricated preconcentration device for breath analysis. Sensors and Actuators B: Chemical 2013, 180 (Supplement C), 130-136.

130. Wang, J.; Moore, J.; Laulhe, S.; Nantz, M.; Achilefu, S.; Kang, K. A., Fluorophore-gold nanoparticle complex for sensitive optical biosensing and imaging. Nanotechnology 2012, 23 (9), 095501.

131. Alhassan, A.; Andersson, J. T., Ketones in Fossil Materials-A Mass Spectrometric Analysis of a Crude Oil and a Coal Tar. Energy \& Fuels 2013, 27 (10), 57705778.

132. Key, J. A.; Li, C.; Cairo, C. W., Detection of Cellular Sialic Acid Content Using Nitrobenzoxadiazole Carbonyl-Reactive Chromophores. Bioconjugate Chemistry 2012, 23 (3), 363-371.

133. Ulrich, S.; Boturyn, D.; Marra, A.; Renaudet, O.; Dumy, P., Oxime Ligation: A Chemoselective Click-Type Reaction for Accessing Multifunctional Biomolecular Constructs. Chemistry - A European Journal 2014, 20 (1), 34-41.

134. Lee, Y. N.; Zhou, X., Method for the determination of some soluble atmospheric carbonyl compounds. Environmental Science \& Technology 1993, 27 (4), 749756.

135. Chi, Y.; Feng, Y.; Wen, S.; Lü, H.; Yu, Z.; Zhang, W.; Sheng, G.; Fu, J., Determination of carbonyl compounds in the atmosphere by DNPH derivatization and LC-ESI-MS/MS detection. Talanta 2007, 72 (2), 539-545.

136. Lary, D. J.; Shallcross, D. E., Central role of carbonyl compounds in atmospheric chemistry. Journal of Geophysical Research: Atmospheres 2000, 105 (D15), 19771-19778.

137. Ajayi, B. P.; Rabindran Jermy, B.; Abussaud, B. A.; Al-Khattaf, S., Oxidative dehydrogenation of n-butane over bimetallic mesoporous and microporous zeolites with CO2 as mild oxidant. J Porous Mater 2013, 20 (5), 1257-1270.

138. Xu, W. Z.; Yang, L.; Charpentier, P. A., Preparation of Antibacterial Softwood via Chemical Attachment of Quaternary Ammonium Compounds Using Supercritical CO2. ACS Sustainable Chemistry \& Engineering 2016, 4 (3), 1551 1561.

139. Alam, T. M.; Dreyer, D. R.; Bielwaski, C. W.; Ruoff, R. S., Measuring Molecular Dynamics and Activation Energies for Quaternary Acyclic Ammonium and Cyclic Pyrrolidinium Ionic Liquids Using 14N NMR Spectroscopy. The Journal of Physical Chemistry A 2011, 115 (17), 4307-4316.

140. Intorp, M.; Purkis, S.; Wagstaff, W., Determination of Carbonyl Compounds in Cigarette Mainstream Smoke. The CORESTA 2010 Collaborative Study and Recommended Method. In Beiträge zur Tabakforschung International/Contributions to Tobacco Research, 2012; Vol. 25, p 361.

141. Biswas, S.; Huang, X.; Badger, W. R.; Nantz, M. H., Nucleophilic cationization reagents. Tetrahedron Letters 2010, 51 (13), 1727-1729.

142. Jensen, C. M.; Lee, D. W., Dry-Ice Bath Based on Ethylene Glycol Mixtures. Journal of Chemical Education 2000, 77 (5), 629. 
143. Fareghi-Alamdari, R.; Ghorbani-Zamani, F.; Shekarriz, M., Surface Passivation of Bare Boron Nanoparticles Using New Dicyanamide-Based Dicationic Ionic Liquid. Energy \& Fuels 2016, 30 (1), 551-559.

144. Etter, J.-F.; Zäther, E.; Svensson, S., Analysis of refill liquids for electronic cigarettes. Addiction 2013, 108 (9), 1671-1679.

145. Ciolino, L. A.; Turner, J. A.; McCauley, H. A.; Smallwood, A. W.; Yi, T. Y., Optimization study for the reversed-phase ion-pair liquid chromatographic determination of nicotine in commercial tobacco products. Journal of Chromatography A 1999, 852 (2), 451-463.

146. Yildiz, D., Nicotine, its metabolism and an overview of its biological effects. Toxicon : official journal of the International Society on Toxinology 2004, 43 (6), 619-32.

147. Pickworth, W. B.; Fant, R. V., Endocrine effects of nicotine administration, tobacco and other drug withdrawal in humans. Psychoneuroendocrinology 1998, $23(2), 131-41$.

148. Benowitz, N. L.; Porchet, H.; Sheiner, L.; Jacob, P., 3rd, Nicotine absorption and cardiovascular effects with smokeless tobacco use: comparison with cigarettes and nicotine gum. Clinical pharmacology and therapeutics 1988, 44 (1), 23-8.

149. Schuller, H. M., Cell type specific, receptor-mediated modulation of growth kinetics in human lung cancer cell lines by nicotine and tobacco-related nitrosamines. Biochemical Pharmacology 1989, 38 (20), 3439-3442.

150. Lambers, D. S.; Clark, K. E., The maternal and fetal physiologic effects of nicotine. Seminars in perinatology 1996, 20 (2), 115-26.

151. Aoshiba, K.; Nagai, A.; Yasui, S.; Konno, K., Nicotine prolongs neutrophil survival by suppressing apoptosis. Journal of Laboratory and Clinical Medicine 1996, 127 (2), 186-194.

152. Mayer, B., How much nicotine kills a human? Tracing back the generally accepted lethal dose to dubious self-experiments in the nineteenth century. Archives of Toxicology 2014, 88 (1), 5-7.

153. Uchiyama, S.; Ohta, K.; Inaba, Y.; Kunugita, N., Determination of Carbonyl Compounds Generated from the E-cigarette Using Coupled Silica Cartridges Impregnated with Hydroquinone and 2,4-Dinitrophenylhydrazine, Followed by High-Performance Liquid Chromatography. Analytical Sciences 2013, 29 (12), 1219-1222.

154. Metcalf, R., Ullmann's Encyclopedia of Industrial Chemistry. 7th ed.; Wiley: 2007.

155. Hashimoto, T.; Mitani, A.; Yamada, Y., Diamine Oxidase from Cultured Roots of Hyoscyamus niger: Its Function in Tropane Alkaloid Biosynthesis. Plant Physiology 1990, 93 (1), 216-221.

156. Dawson, R. F.; Christman, D. R.; Anderson, R. C.; Solt, M. L.; D'Adamo, A. F.; Weiss, U., BIOSYNTHESIS OF THE PYRIDINE RING OF NICOTINE1. Journal of the American Chemical Society 1956, 78 (11), 2645-2646.

157. Yang, K. S.; Gholson, R. K.; Waller, G. R., Studies on Nicotine Biosynthesis1. Journal of the American Chemical Society 1965, 87 (18), 4184-4188.

158. Katoh, A.; Ohki, H.; Inai, K.; Hashimoto, T., Molecular regulation of nicotine biosynthesis. Plant Biotechnology 2005, 22 (5), 389-392. 
159. Tayyarah, R.; Long, G. A., Comparison of select analytes in aerosol from ecigarettes with smoke from conventional cigarettes and with ambient air. Regulatory Toxicology and Pharmacology 2014, 70 (3), 704-710.

160. Trehy, M. L.; Ye, W.; Hadwiger, M. E.; Moore, T. W.; Allgire, J. F.; Woodruff, J. T.; Ahadi, S. S.; Black, J. C.; Westenberger, B. J., ANALYSIS OF ELECTRONIC CIGARETTE CARTRIDGES, REFILL SOLUTIONS, AND SMOKE FOR NICOTINE AND NICOTINE RELATED IMPURITIES. Journal of Liquid Chromatography \& Related Technologies 2011, 34 (14), 1442-1458.

161. El-Hellani, A.; El-Hage, R.; Baalbaki, R.; Salman, R.; Talih, S.; Shihadeh, A.; Saliba, N. A., Free-Base and Protonated Nicotine in Electronic Cigarette Liquids and Aerosols. Chemical Research in Toxicology 2015, 28 (8), 1532-1537.

162. Pendergrass, S. M.; Krake, A. M.; Jaycox, L. B., Development of a Versatile Method for the Detection of Nicotine in Air. AIHAJ - American Industrial Hygiene Association 2000, 61 (4), 469-472.

163. Djordjevic, M. V.; Stellman, S. D.; Zang, E., Doses of Nicotine and Lung Carcinogens Delivered to Cigarette Smokers. JNCI: Journal of the National Cancer Institute 2000, 92 (2), 106-111.

164. Adams, J. D.; Lee, S. J.; Vinchkoski, N.; Castonguay, A.; Hoffmann, D., On the formation of the tobacco-specific carcinogen 4-(methylnitrosamino)-1-(3pyridyl)-1-butanone during smoking. Cancer Letters 1983, 17 (3), 339-346.

165. Rosado-Reyes, C. M.; Tsang, W., Thermal Stability of Larger Carbonyl Compounds: 2-Methylbutyraldehyde. International Journal of Chemical Kinetics 2014, 46 (5), 285-293. 


\title{
CURRICULUM VITAE
}

\author{
Mumiye A. Ogunwale \\ 822 Melford Avenue apt 2 \\ Louisville, KY 40217 \\ mumiye828@yahoo.com \\ Mobile: (502) 819-8130
}

\section{ACADEMIC \& WORK EXPERIENCE}

University of Louisville, Louisville, KY

Graduate Research Assistant
August 2012 -Present

- Method development, derivatization, analysis and quantification of trace-level volatile organic compounds (VOCs) using Fourier transform ion cyclotron mass spectrometry, GC-MS, NMR, and FT-IR

- Wafer processing including photolithography, alignment, deep reactive ion etching, wet etching and thermal oxidation

- Multi-step organic synthesis of chemoselective reagents for the capture of trace volatile aldehydes and ketones in complex mixtures, such as breath, cigarette and e-cigarette aerosols

- Maintain laboratory instruments/equipment; enforce University chemical lab safety practices

- Coordinate and manage group research activities; develop and execute laboratory work plans/schedules

- Maintain high quality laboratory documentation

Tennessee State University, Nashville, TN

August 2009- December 2011

Graduate Teaching Assistant

- Supervision of undergraduate laboratory courses in general Chemistry

- Synthesis, characterization, and analysis of novel compounds having biological activity

\section{Coates Brothers (WA) Ltd, Lagos Nigeria}

Coatings Chemist

- Color matching and formulations of polyester-based coatings

- Support scale-up from lab to production scale, including process development

- Quality control, rheology of polyester-based coatings and varnishes, measurements of dispersion, viscosity, gloss, corrosion resistance, scratch test, fastness and bleeding of coating inks

- Certifying production output to make sure that all the quality specifications were met for packaging

- Technical services involving troubleshooting for coating products

- Writing detailed reports and maintaining high quality laboratory documentation 


\section{SKILLS}

\section{Computer \& Technology Skills}

- ChemDraw, Microsoft Office

- Databases: SciFinder Scholar, Reaxys

\section{Instrumentation}

- Spectroscopy: GC-MS, NMR, FT-IR, FT-ICR-MS

- Microfabrication: Photolithography, Front and back optical alignment, Deep Reactive Ion Etching (DRIE), Wet etching

- Metrology: Dektak surface profilermeter, Ellipsometry

\section{EDUCATION \& RESEARCH}

\section{University of Louisville, KY}

August 2012- November 2017

- Philosophy in Chemistry Dissertation (in progress): Chemoselective reagents for capture of trace-level volatile aldehydes and ketones in breath using a microchip approach

Advisors: Michael H. Nantz, PhD (Chemistry) \& Xiao-An Fu, PhD (Chemical Engineering)

\section{Tennessee State University, Nashville, TN}

August 2009 - December 2011

- MS Chemistry Thesis: Synthesis of new fluorinated hexahydroquinoline derivatives as potential calcium channel modulators

- Advisor: Cosmas O. Okoro, $\mathrm{PhD}$

Federal University of Technology, Akure, Nigeria

- B. Tech. (Hons) Industrial Chemistry Project: The Chemical composition and mineral content of sorghum bicolor L stem used as a color additive in food

Advisor: Abayomi Adetuyi, $\mathrm{PhD}$

\section{PUBLICATIONS}

- Mumiye A. Ogunwale, Mingxiao Li, Mandapati V. Ramakrishnam Raju, Yizheng Chen, Michael H. Nantz, Daniel J. Conklin and Xiao-An Fu. Aldehyde detection in electronic cigarette aerosols. ACS Omega 2017, 2, 1207-1214.

- Mumiye A. Ogunwale, Yizheng Chen, Whitney S. Theis, Michael H. Nantz, Daniel J. Conklin and Xiao-An Fu. A novel method of nicotine quantification in electronic cigarette liquids and aerosols. Anal. Methods 2017, 9, 4261-4266.

- Mumiye A. Ogunwale, Daniel J. Conklin, Yizheng Chen, Michael H. Nantz, XiaoAn Fu, Daniel W. Riggs, Pawel Lorkiewicz, Aruni Bhatnagar, and Sanjay Srivastava. Electronic cigarette-generated aldehydes: The contribution of e-liquid components to their formation and the use of urinary aldehyde metabolites as biomarkers of exposure. Aerosol Science \& Technology (Submitted) 
- Mumiye A. Ogunwale, Xiao-An Fu and Michael H. Nantz. 2 Hydrazinyl N, N, Ntrimethylethan ammonium iodide: a chemoselective reagent for isolation of volatile aldehydes and ketones using a microreactor approach. (Manuscript in preparation for Analyst).

- Mumiye A. Ogunwale, Ralph J. Knipp, Michael H. Nantz, and Xiao-An Fu. Reaction kinetics of quaternary ammonium aminooxy compounds with carbonyl compounds. (Manuscript in preparation for ACS Physical Chemistry A)

- Cosmas O. Ok̄oro, Mumiye A. Ogunwale and Tasneem Siddiquee. Synthesis of Some New Fluorinated Hexahydroquinoline and Acridinedione Derivatives in Trifluoroethanol. Appl. Sci. 2012, 2(2), 368- 374.

\section{CONFERENCE PRESENTATIONS}

- Mumiye A. Ogunwale; Xiao-An Fu; Michael H. Nantz. "2-Hydrazinyl-N, N, Ntrimethylethan-1- ammonium iodide: A reagent for chemoselective isolation of volatile aldehydes and ketones from exhaled breath" 253 ACS National Meeting \& Exposition, San Francisco, CA, (Oral, final paper number: ANYL 468), April 2017

- Mumiye A. Ogunwale; Yizheng Chen; Whitney S. Theis; Michael H. Nantz; Daniel J. Conklin; Xiao-An Fu. "Nicotine in Electronic Liquids and aerosols" NIH-FDA TCORS Grantee Meeting, Bethesda, MD, November, 2016 (Poster)

- Mumiye A. Ogunwale; Mingxiao Li; Mandapati V. Ramakrishnam Raju; Michael H. Nantz; Daniel J. Conklin; Xiao-An Fu "Aldehyde Detection in Electronic Cigarette Aerosols" ACS 2016 Central Regional Meeting (CERM), Covington, KY, May 2016 (Poster)

- Mumiye A. Ogunwale; Mingxiao Li; Mandapati V. Ramakrishnam Raju; Michael H. Nantz; Daniel J. Conklin; Xiao-An Fu. "Detection of carbonyl Compounds in Ecigarettes Vapor” NOBCChE Annual Conference, September 2015 (Poster)

- Mumiye A. Ogunwale and Cosmas O. Okoro. "Synthesis of Novel Trifluoromethylated Hexahydroquinoline Derivatives as Potential Calcium Channel Modulators" 41st ACS National Meeting \& Exposition, Anaheim, CA, United States, MEDI-142 (Poster), March 2011

\section{AWARDS}

- Institute for Molecular Diversity \& Drug Design (IMD3), University of Louisville Travel Award (April, 2017)

- Graduate Research Assistantship (August 2014 to date)

- NOBCChE Advancing Science Travel Grant (August 2015)

- University of Louisville Pre doctoral Fellowship award (August 2012- July 2014)

- RCCG Agape House, Nashville, Tennessee Initiative for Academic Excellence Scholarship Award (2009)

- Federal Government (Nigeria) Academic Excellence Scholarship Award (2001/2002 Academic session) 


\section{CERTIFICATION}

- $\quad$ Six Sigma Green Belt (Institute of Industrial and Systems Engineers) -August 2017

\section{PROFESSIONAL ASSOCIATION}

- American Chemical Society (member)

- Society for Research on Nicotine and Tobacco (member)

- National Organization for Professional Advancement of Black Chemists and Chemical Engineer NOBCChE (Member)

\section{REFERENCES}

Prof. Michael H. Nantz

Department of Chemistry

University of Louisville, KY 40208

(502) 852-8069

michael.nantz@louisville.edu

Prof. Xiao-An Fu

Department of Chemical Engineering

University of Louisville, KY 40208

xiaoan.fu@louisville.edu
Prof. Nantali B. Richter Department of Chemistry University of Louisville, KY 40208 (502) 852-2733

natali.richter@louisville.edu

Prof. Cosmas O. Okoro

Department of Chemistry

Tennessee State University, TN 37209

cokoro@Tnstate.edu 